\title{
An Ifnar1 allele impairs the colonization of gut bacteria and promotes tuberculosis
}

\section{Lingming Chen}

Department of Microbiology, Zhongshan School of Medicine, Key Laboratory for Tropical Diseases Control of the Ministry of Education, Sun Yat-sen University, Guangzhou 510080, China.

\section{Guoliang Zhang}

National Clinical Research Center for Infection Diseases, Guangdong Key Laboratory for Emerging Infectious Diseases, Shenzhen Third People's Hospital, Southern University of Science and Technology,

\section{Guobao Li}

National Clinical Research Center for Infection Diseases, Guangdong Key Laboratory for Emerging Infectious Diseases, Shenzhen Third People's Hospital, Southern University of Science and Technology,

\section{Wei Wang}

Department of Clinical Laboratory, Foshan Fourth People's Hospital, Foshan 528000, China.

\section{Zhen-Huang Ge}

Sun Yat-sen University

\section{Yi Yang}

Department of Microbiology, Zhongshan School of Medicine, Key Laboratory for Tropical Diseases Control of the Ministry of Education, Sun Yat-sen University, Guangzhou 510080, China.

\section{Xing He}

National Clinical Research Center for Infection Diseases, Guangdong Key Laboratory for Emerging Infectious Diseases, Shenzhen Third People's Hospital, Southern University of Science and Technology,

\section{Zhi Liu}

National Clinical Research Center for Infection Diseases, Guangdong Key Laboratory for Emerging Infectious Diseases, Shenzhen Third People's Hospital, Southern University of Science and Technology,

\section{Zhiyi Zhang}

Department of Microbiology, Zhongshan School of Medicine, Key Laboratory for Tropical Diseases Control of the Ministry of Education, Sun Yat-sen University, Guangzhou 510080, China.

\section{Qiongdan Mai}

Department of Microbiology, Zhongshan School of Medicine, Key Laboratory for Tropical Diseases Control of the Ministry of Education, Sun Yat-sen University, Guangzhou 510080, China.

\section{Yiwei Chen}

Department of Microbiology, Zhongshan School of Medicine, Key Laboratory for Tropical Diseases Control of the Ministry of Education, Sun Yat-sen University, Guangzhou 510080, China.

\section{Zixu Chen}


Department of Microbiology, Zhongshan School of Medicine, Key Laboratory for Tropical Diseases Control of the Ministry of Education, Sun Yat-sen University, Guangzhou 510080, China.

\section{Jiang Pi}

Department of Microbiology and Immunology, Center for Primate Biomedical Research, University of Illinois College of Medicine, Chicago, IL 60612, USA

\section{Shuai Yang}

Key Laboratory of Gene Function and Regulation of the Ministry of Education, State Key Laboratory of Biocontrol, School of Life Sciences, Sun Yat-sen University, Guangzhou, 510006, Guangdong, China Jun Cui

Key Laboratory of Gene Function and Regulation of the Ministry of Education, State Key Laboratory of Biocontrol, School of Life Sciences, Sun Yat-sen University, Guangzhou, 510006, Guangdong, China

\section{Haipeng Liu}

Shanghai Pulmonary Hospital, Tongji University School of Medicine

\section{Ling Shen}

Department of Microbiology and Immunology, Center for Primate Biomedical Research, University of Illinois College of Medicine, Chicago, IL 60612, USA

\section{Lingchan Zeng}

Clinical Research Center, Department of Medical Records Management, Guanghua School of Stomatology, Hospital of Stomatology, Sun Yat-sen University, Guangzhou, Guangdong, China.

\section{Lin Zhou}

Guangdong Center for Tuberculosis Control, National Clinical Research Center for Tuberculosis, Guangzhou 510430, China

\section{Xinchun Chen}

Department of Pathogen Biology, Shenzhen University School of Medicine, Shenzhen, Guangdong 518060, China

\section{Baoxue Ge}

Shanghai Pulmonary Hospital https://orcid.org/0000-0002-4086-8299

\section{Zheng W. Chen}

Department of Microbiology and Immunology, Center for Primate Biomedical Research, University of Illinois College of Medicine, Chicago, IL 60612, USA

\section{Gucheng Zeng ( $\square$ zenggch@mail.sysu.edu.cn )}

Department of Microbiology, Zhongshan School of Medicine, Key Laboratory for Tropical Diseases Control of the Ministry of Education, Sun Yat-sen University, Guangzhou 510080, China

\section{Article}

Keywords: tuberculosis, host genetics, gut bacteria, disease susceptibility 
DOl: https://doi.org/10.21203/rs.3.rs-847439/v1

License: (c) (1) This work is licensed under a Creative Commons Attribution 4.0 International License. Read Full License

Version of Record: A version of this preprint was published at Nature Metabolism on March 14th, 2022. See the published version at https://doi.org/10.1038/s42255-022-00547-3. 
3 Lingming Chen ${ }^{1, \#}$, Guoliang Zhang ${ }^{2, \#}$, Guobao Li $^{2, \#}$, Wei Wang ${ }^{3, \#}$, Zhenhuang Ge ${ }^{4, \#}$,

4 Yi Yang ${ }^{1}$, Xing $\mathrm{He}^{2}$, Zhi Liu ${ }^{2}$, Zhiyi Zhang ${ }^{1}$, Qiongdan $\mathrm{Mai}^{1}$, Yiwei $\mathrm{Chen}^{1}$, Zixu

5 Chen ${ }^{1}$, Jiang $\mathrm{Pi}^{5}$, Shuai Yang ${ }^{6}$, Jun Cui ${ }^{6}$, Haipeng Liu ${ }^{7}$, Ling Shen ${ }^{5}$, Lingchan Zeng ${ }^{8}$,

6 Lin Zhou ${ }^{9}$, Xinchun Chen ${ }^{10}$, Baoxue Ge ${ }^{7}$, Zheng W. Chen ${ }^{5}$, Gucheng Zeng ${ }^{1 *}$

\section{An Ifnar1 allele impairs the colonization of gut bacteria} and promotes tuberculosis

1. Department of Microbiology, Zhongshan School of Medicine, Key Laboratory for

Tropical Diseases Control of the Ministry of Education, Sun Yat-sen University, Guangzhou 510080, China.

2. National Clinical Research Center for Infection Diseases, Guangdong Key Laboratory for Emerging Infectious Diseases, Shenzhen Third People's Hospital, Southern University of Science and Technology, Shenzhen 518112, China.

3. Department of Clinical Laboratory, Foshan Fourth People's Hospital, Foshan 528000, China.

4. School of Chemistry, Sun Yat-sen University, Guangzhou 510275, China.

5. Department of Microbiology and Immunology, Center for Primate Biomedical Research, University of Illinois College of Medicine, Chicago, IL 60612, USA.

6. Key Laboratory of Gene Function and Regulation of the Ministry of Education, State Key Laboratory of Biocontrol, School of Life Sciences, Sun Yat-sen University, Guangzhou, 510006, Guangdong, China.

7. Shanghai Key Lab of Tuberculosis, Shanghai Pulmonary Hospital, School of 
Medicine, Tongji University, Shanghai, China.

Phone: +86-13610059833

* Corresponding author

8. Clinical Research Center, Department of Medical Records Management, Guanghua School of Stomatology, Hospital of Stomatology, Sun Yat-sen University, Guangzhou, Guangdong, China.

9. Guangdong Center for Tuberculosis Control, National Clinical Research Center for Tuberculosis, Guangzhou 510430, China.

10. Department of Pathogen Biology, Shenzhen University School of Medicine, Shenzhen, Guangdong 518060, China.

\# These authors contributed equally to this work.

Address: Department of Microbiology, Zhongshan School of Medicine,

Sun Yat-sen University, Guangzhou 510080, China

E-mail: zenggch@mail.sysu.edu.cn 


\section{Abstract}

Both host genetics and gut microbiome have important effects on human health, yet how host genetics regulates gut bacteria and further determines disease susceptibility remains unclear. Here, we find that gut microbiome pattern of active tuberculosis (TB) patients is characterized by a reduction of core species found across healthy controls, particularly Akkermansia muciniphila (A. muciniphila). Oral treatments of $A$. muciniphila or palmitoleic acid, an A. muciniphila-derived metabolite, strongly inhibit TB infection through epigenetically inhibiting TNF- $\alpha$. We use three independent cohorts comprising 6512 individuals and identify that single-nucleotide polymorphism rs2257167 "G" allele of type I interferon (IFN-I) receptor 1 (Ifnarl) contributes to stronger IFN-I signaling, impaired colonization and abundance of $A$. muciniphila, reduced production of palmitoleic acid, higher TNF- $\alpha$, and much severer TB disease in humans and transgenic mice. Thus, host genetics are critical in modulating structure and functions of gut microbiome and gut microbial metabolites, which further determines disease susceptibility. 


\section{Main}

Gut microbial dysbiosis is linked to the development of intra- and extra-intestinal diseases ${ }^{1,2}$. The structures and functions of human gut microbiome are now believed mainly to be shaped by environmental factors ${ }^{3}$ and diet ${ }^{4-7}$. However, both innate and adaptive immune components are becoming increasingly critical in regulating the colonization, composition, and abundance of gut microbiota ${ }^{8-10}$. Thus, the alteration of structure and function of gut microbiome should be the result of bi-directional interactions between host factors and its microbial composition and abundance ${ }^{8-10}$, and it is unlikely that host genetics only play a redundant or less important role in determining the structure and function of gut microbiota ${ }^{11-15}$.

Tuberculosis (TB), a severe infectious disease with extremely high morbidity and mortality, is caused by an airborne bacterial pathogen, Mycobacterium tuberculosis (M. tuberculosis) ${ }^{16}$. Susceptibility to M. tuberculosis infection is affected by multiple intrinsic and extrinsic factors driving host-pathogen interactions. Accumulating evidence has shown that changes in the gut microbiota's structure and activity encode a broad array of functions associated with lung diseases $2,17,18$, which is now commonly referred to as the "gut-lung axis" 19-21. While immune deficiencies or disorders represent key rationales leading to severe and recurrent $\mathrm{TB}$ infections, the excessive inflammatory response may be a cause of exacerbated TB during $M$. tuberculosis infection ${ }^{22-26}$. Therefore, regulating immune homeostasis of the "gutlung axis" may serve as a key approach for successful TB control. Although it was shown that gut microbiota aberration might correlate with TB pathogenesis ${ }^{27-29}$, it is 
still unclear whether and how host genetics influence the structure and function of gut microbiome to maintain immune homeostasis of "gut-lung axis" and therefore determine the variable TB pathogenicity and susceptibility in M. tuberculosis-infected individuals.

In this study, we focused on investigating the relationship among inter-individual variations in host genetic factors, gut microbial abundance, and inflammatory responses in influencing susceptibility and severity of TB infection. We identified a single-nucleotide polymorphism (SNP) in the type I interferon (IFN-I) receptor (Ifnarl) gene from 3,125 active TB patients (TB) and 3,387 healthy controls (HC) in three independent cohorts. Importantly, we found that Ifnarl rs2257167 G allele could enhance IFN-I signaling, impair the intestinal colonization of A. muciniphila, reduce the production of an $A$. muciniphila-derived metabolite, palmitoleic acid, increase production of pro-inflammatory TNF- $\alpha$, and promote TB infection in both humans and transgenic mouse models, and oral administrations of A. muciniphila or palmitoleic acid could reduce TB infections in $M$. tuberculosis-infected mouse models. This work reveals that compositional structures and functions of gut microbiome and gut microbial metabolites are partially determined by host genetic factors, which could have translational potentials for new prevention strategies and therapeutics in TB and potentially other diseases.

\section{Results}


within gut bacteria, particularly $A$. muciniphila.

As an initial step to investigate the relationship among host genetic factors, gut microbiota, and disease susceptibility, we performed $16 \mathrm{~S}$ rDNA sequencing on fecal samples to assess the features of the gut bacteria in healthy controls (HC) and active TB patients (TB). Since the composition and abundance of gut microbiota might be affected by environmental factors and dietary habits ${ }^{3,4}$, faeces were collected in two independent cohorts (Supplementary Table 1). There were significant differences of gut bacteria communities in the fecal contents of HC and TB (Fig. 1a; Extended

\section{Data Fig.1a).}

Notably, compared to HC, TB patients showed a broad reduction in diversity and abundance of gut bacteria species (Fig. 1b; Extended Data Fig. 2a). Particularly, Verrucomicrobia was the only phylum with reduced levels in both two cohorts (Extended Data Fig. 1b, 2b), and A. muciniphila, a representative species member of Verrucomicrobia phylum ${ }^{30}$, was repeatedly observed in two different cohorts to show reduced species richness (Extended Data Fig. 1c, 2c). Also, the analysis of top 10 species highly enriched in $\mathrm{HC}$ or $\mathrm{TB}$ showed that $A$. muciniphila was the only bacterial species with reduced abundance in TB patients in both two cohorts (Fig. 1cd; Extended Data Fig. 2d). Importantly, A. muciniphila was a critical bacterial species played a significant role in contributing to the abundance of the entire gut microbiome in both HC and TB (Extended Data Fig. 1d, 2e).

Since compositional shifts in the microbiota, such as reduced microbial diversity and abundance, may confer negative effects on microbiota's functionality, random 
forest analysis ${ }^{31}$ was performed to further investigate the contribution of the top 10 most-reduced species in TB patients, and found that A. muciniphila displayed the confirmed importance Z-score (Fig. 1e; Extended Data Fig. 2f). In addition, such abundance reduction of A. muciniphila in TB patients was consistent with the altered abundance of A. muciniphila observed in another study ${ }^{32}$.

To further verify the random forest analysis, LEfSe analysis was carried out. Several bacteria including B. vulgatus, Bacteroides uniformis, Erysipelatoclostridium ramosun, A. muciniphila, Bacteroides caccae, and Parabacteroides merdae were found to have strong influences on compositional shifts of HC or TB (Extended Data Fig. 1e, 2g). Additionally, A. muciniphila was the most significantly upregulated bacterial species in $\mathrm{HC}$, and B. vulgatus showed the largest proportion among these six bacteria (Extended Data Fig. 1f, 2h-i). Therefore, these results collectively suggested that reduced abundance of A. muciniphila or B. vulgatus was highly associated with the development of active TB.

\subsection{A. muciniphila confers anti-TB protection via inhibiting production of pro-} inflammatory TNF- $\alpha$.

$$
\text { We then examined whether loss or reduction of the abundance of } A \text {. muciniphila or }
$$
B. vulgatus might be correlated with abnormal inflammation statuses in active TB. Six major Th1/Th2 cytokines (TNF- $\alpha /$ IFN- $\gamma /$ IL-10/IL-2/IL-4/IL-6) were selected for analyses based on their well-established importance in mediating TB susceptibility and severity ${ }^{33-38}$. Ex vivo production of TNF- $\alpha /$ IFN- $\gamma$ was higher in TB than those of HC, and such increased production of TNF- $\alpha /$ IFN- $\gamma /$ IL- 10 was at least partially $M$. 
tuberculosis-specific as ex vivo re-stimulation using lysates of $M$. tuberculosis further enhanced their productions (Fig. 1f-g). Interestingly, the abundance of A. muciniphila was negatively correlated with TNF- $\alpha$ production (Fig. 1h). However, there was an erratic association between A. muciniphila abundance and IFN- $\gamma$ (Fig. 1i) or IL-10 response (Fig. 1j), and also no significant correlation between $B$. vulgatus abundance and TNF- $\alpha /$ IFN- $\gamma /$ IL-10 production (Extended Data Fig. 3). Collectively, these results indicated that a higher TNF- $\alpha$ response was linked to the decreased abundance of A. muciniphila but not B. vulgatus in M. tuberculosis infection, highlighting that $A$. muciniphila might function to regulate $\mathrm{TB}$ susceptibility or severity via regulating TNF- $\alpha$ response.

We then investigated potential in vivo effect of A. muciniphila and B. vulgatus during M. tuberculosis infection. An aerosol M. tuberculosis infection model ${ }^{39}$ was developed, and antibiotics-treated mice exhibited much severer pathological impairment and much higher bacillus burdens in lungs (Extended Data Fig. 4a-e), confirming that gut microbiota was necessary for mediating anti-TB protection. Moreover, M. tuberculosis-infected mice were orally treated with A. muciniphila or B. vulgatus, and it was mice gavaged with $A$. muciniphila but not $B$. vulgatus showed much less hemorrhage and pathological impairment in lungs (Fig. 2a-f) and exhibited lower pulmonary bacillus burdens (Fig. 2g-h). Additionally, it showed that $M$. tuberculosis-infected mice with A. muciniphila but not B. vulgatus treatments exhibited significantly lower levels of TNF- $\alpha$, but not IFN- $\gamma /$ IL-2/IL-4/IL-6/IL-10 (Fig. 2i; Extended Data Fig. 6a). However, such anti-TB protection effects of $A$. 
muciniphila in M. tuberculosis-infected mice were partially compromised with TNF- $\alpha$ signaling blockade (Extended Data Fig. 5a-e). Thus, these data collectively suggested that A.muciniphila could confer anti-TB protection via modulating TNF- $\alpha$ signaling, and such protection effects required intact host TNF- $\alpha$ signaling.

\subsection{Impaired colonization of $A$. muciniphila reduces production of its metabolite,} palmitoleic acid, and dietary palmitoleic acid inhibits TNF- $\alpha$ production and confers strong protection against TB infection.

We then investigated what bacterial components of A. muciniphila mediated antiTB effects. Metabolite signatures of bacterial cell components and culture supernatants of A. muciniphila were subjected to GC-TOF-MS and random forest analyses. It showed that A. muciniphila-derived 2-Hydroxybutanoic acid, 3Hydroxybutyric acid, and palmitoleic acid were potential dominant metabolites involved in mediating anti-TB immunity (Fig. 3a-b). However, only palmitoleic acid but not 2-Hydroxybutanoic acid and 3-Hydroxybutyric acid could significantly reduce TNF- $\alpha$ production (Fig. 3c; Extended Data Fig. 6c). Consistently, KEGG analysis revealed that $A$. muciniphila was less likely to express long-chain-fatty-acid-CoA ligase than B. vulgatus (Extended Data Fig. 7a-e) and the expression of essential FAT gene were significantly increased in feces of mice with $A$. muciniphila treatment (Extended Data Fig. 7f), which might in together allow more production and accumulation of palmitoleic acid in A. muciniphila.

Furthermore, oral administrations of A. muciniphila, but not B. vulgatus, 
significantly increased palmitoleic acid concentrations in plasma of mice (Fig. 3d). Oral administrations of live but not inactivated A. muciniphila significantly increased palmitoleic acid concentrations in both plasma and feces of mice (Fig. 3e, f). Moreover, KEGG analysis showed that other gut bacteria, which displayed significantly differential abundance between $\mathrm{HC}$ and $\mathrm{TB}$, could not produce or accumulate palmitoleic acid as like A. muciniphila (Extended Data Fig. 7g-j). These data collectively indicated that intestine-colonized A. muciniphila was a most important contributor to higher palmitoleic acid levels.

Importantly, the relative concentration of palmitoleic acid were significantly higher in both feces and plasma from HC than those from TB (Fig. 3g-h), indicating that decreased palmitoleic acid concentration was correlated with active TB in humans. Indeed, M. tuberculosis-infected mice with dietary treatments of palmitoleic acid but not butyrate showed much less pulmonary unresolved hemorrhage and pathological impairment (Fig. 4a-d) and exhibited lower pulmonary bacillus burdens (Fig. 4e-f). Consistently, there was no significant difference in butyrate concentrations in feces between $\mathrm{HC}$ and TB (Fig. 3g), suggesting that palmitoleic acid might play a more important role than butyrate in mediating TB protection or susceptibility. Additionally, M. tuberculosis-infected mice with dietary palmitoleic acid showed significantly lower expression of TNF- $\alpha$ but not IFN- $\gamma /$ IL-2/IL-4/IL-6/IL-10 in serum compared to butyrate or water treatment (Fig. 4g; Extended Data Fig. 6b). These results indicate that such a palmitoleic acid-mediated inhibition effect against pro-inflammatory TNF$\alpha$ is a key mechanism for A. muciniphila-conferred anti-TB protective immunity. 

signaling.

IFN-I signaling plays critical roles in determining TB pathogenicity, intestinal epithelium development and homeostasis of gut microbial ecosystem ${ }^{40-45}$. Thus, we hypothesized that IFN-I signaling affected the abundance of A. muciniphila and in turn resulted in varied TB susceptibility and severity. We fed wild-type and Ifnar $1^{-/-}$ mice with feces from HC and TB, respectively (Extended Data Fig. 8a), and found that Ifnar $^{-/-}$mice harbored approximately 2-6 times more abundance of anaerobic bacteria in jejunum or cecum compared to wild-type mice (Extended Data Fig. 8b). Interestingly, regardless of whether feces came from $\mathrm{HC}$ or from $\mathrm{TB}$, A. muciniphila exhibited much higher levels of colonization and abundance in jejunum, ileum, and cecum of Ifnarl ${ }^{-/}$mice compared to wild-type controls (Extended Data Fig. 8c). Together, these results suggested that deletion or reduction of IFN-I signaling favored intestinal colonization and abundance of A. muciniphila.

\subsection{Ifnar1 rs2257167 $\mathrm{G}$ allele dictates stronger IFN-I signaling and increases the} risk of developing active TB in humans.

We further postulated that there might be host genetic variants present in Ifnarl gene that altered the strength of IFN-I signaling and resulted in inter-individual variations in the abundance and immune regulation function of $A$. muciniphila and $A$. muciniphila-derived metabolites during M. tuberculosis infection. Targeted sequencing was performed to analyze the human Ifnarl gene in a discovery cohort in 
Guangzhou, China (Supplementary Table 2). Nine SNPs in the intron and only one SNP rs2257167 (which would result in a valine-to-leucine substitution at codon 168 in human IFNAR1 (termed as hIFNAR1-V168L) (Extended Data Fig. 10)) in the exon, were identified (Supplementary Table 3). Since Ifnar1 rs2257167 GG and GC as well as rs1041868 GG and GA genotypes showed the most significant trend toward higher risk of developing active TB in the Guangzhou cohort (Supplementary Table 3), two larger independent cohorts, including Shenzhen $(\mathrm{HC}=1445, \mathrm{~TB}=1533)^{46}$ and Foshan cohort $(\mathrm{HC}=1679, \mathrm{~TB}=1328)$ in China were recruited to further investigate whether $\mathrm{rs} 2257167 \mathrm{G}$ or $\mathrm{rs} 1041868 \mathrm{G}$ was indeed a risk allele of developing active TB or much severer TB (Supplementary Table 2). Indeed, further sequencing analysis 47 validated that individuals carrying rs $2257167 \mathrm{G}$ allele exhibited significantly increased risk of developing active TB in both Shenzhen and Foshan cohorts ( Supplementary Table 4).

Notably, we also discovered that individuals carrying rs2257167 genotypes GG and GC displayed higher expression levels of phospho (p)-STAT1, IFN- $\beta$ and ISG15 than those carrying genotype CC (Fig. 5a; Extended Data Fig. 11a-c), suggesting that the $\mathrm{G}$ allele enhanced IFN-I signaling. To confirm it, we developed two transgenic mouse lines, denoted as IFNAR1-V168V (harboring $\mathrm{G}$ allele) and IFNAR1-V168L (harboring $\mathrm{C}$ allele), respectively (Extended Data Fig. 12a). There was no significant difference in gene copies or expression levels of hlfnarl between IFNAR1-V168V and IFNAR1-V168L mice (Extended Data Fig. 12b-c). However, the expression of p-STAT1, IFN- $\beta$ and $I S G 15$ in BMDM were higher in IFNAR1-V168V mice than in 
IFNAR1-V168L mice (Fig. 5b; Extended Data Fig. 11d-e). Furthermore, the levels of p-STAT1 in intestinal epitheliums as well as IFN- $\beta$ protein in serum were higher in IFNAR1-V168V mice than in IFNAR1-V168L mice, but these differences weren't observed in the lungs during M. tuberculosis infection (Extended Data Fig. 11f-g). Additionally, IFNAR1-V168V mice showed higher expression of IFN- $\beta$ and ISG1 in BMDM and intestinal epitheliums, but not in the lungs, compared to IFNAR1-V168L mice (Extended Data Fig. 11h-I). Collectively, these results suggested that the Ifnarl rs2257167 G allele enhanced IFN-I signaling in both humans and mouse models.

\subsection{Ifnar1 $\operatorname{rs} 2257167 \mathrm{G}$ allele decreases the intestinal abundance of $A$.} muciniphila, reduces productions of $A$. muciniphila-derived palmitoleic acid, and promotes $\mathrm{TB}$ infection.

To explore whether Ifnarl rs2257167 G allele affected the intestinal colonization and abundance of $A$. muciniphila as well as production of $A$. muciniphila-derived palmitoleic acid, IFNAR1-V168L and IFNAR1-V168V mice with antibiotics pretreatment were orally given with the same amounts of $A$. muciniphila (Fig. 5c). IFNAR1-V168V mice indeed harbored less A. muciniphila in intestinal epitheliums, compared to their IFNAR1-V168L counterparts (Fig. 5d-g; Extended Data Fig. 13). Importantly, IFNAR1-V168V mice contained lower concentration levels of palmitoleic acid in plasma than IFNAR1-V168L mice did after A. muciniphila treatment (Fig. 5h). These findings collectively revealed that Ifnarl rs2257167 G allele dictating stronger IFN-I signaling could impede the intestinal abundance of $A$. 
muciniphila and reduce palmitoleic acid levels.

Meanwhile, there were significantly lower A. muciniphila abundance and palmitoleic acid concentration in both fecal samples and plasma in M. tuberculosisinfected IFNAR1-V168V mice, compared to IFNAR1-V168L mice (Fig. 5i-j). IFNAR1-V168V mice exhibited larger-scale pathological impairment and more infiltration of inflammatory cells (Fig. 5k-m), higher pulmonary bacillus burdens (Fig. 5n-o) and TNF- $\alpha$ production than IFNAR1-V168L mice during M. tuberculosis infection (Fig. 5p; Extended Data Fig. 6d). We also analyzed whether increased $A$. muciniphila abundance could reverse the Ifnarl rs2257167 G allele-induced TB severity. Interestingly, A. muciniphila treatment showed consistent protective effects against M. tuberculosis infection (Fig. 6a-d; Extended Data Fig. 14a) and significantly lower levels of TNF- $\alpha$ in IFNAR1-V168V mice (Fig. 6e; Extended Data Fig. 14b). Dietary palmitoleic acid alleviated pulmonary TB pathology, reduced bacillus burdens and TNF- $\alpha$ production in M. tuberculosis-infected IFNAR1-V168V mice (Fig. 6f-j; Extended Data Fig. 14c). These results suggested that oral administrations of A. muciniphila or palmitoleic acid could reverse Ifnarl rs2257167 G allele-induced TB severity and confer anti-TB protection in a genotype-specific manner.

2.7 Ifnar1 rs2257167 $\mathrm{G}$ allele is associated with less $A$. muciniphila abundance, higher TNF- $\alpha$ production, severer TB pathology, and poorer prognosis in patients with active $T B$. 
We then investigated whether Ifnarl $\operatorname{rs} 2257167 \mathrm{G}$ allele could affect $A$.

muciniphila abundance in active human TB. TB patients carrying genotypes GC and GG possessed a significantly lower abundance of $A$. muciniphila than patients carrying genotype CC (Fig. 7a-b). Importantly, the relative concentration of palmitoleic acid were significantly lower in both feces and plasma from TB patients carrying Ifnarl rs2257167 GC and GG than carriers of genotypes CC (Fig. 7c-d). Additionally, compared to TB patients carrying Ifnarl rs2257167 CC, carriers of genotypes GC and GG showed much higher (approximately 5-6 folds) ex vivo expression levels of M. tuberculosis-specific TNF- $\alpha$ (Fig. 7e-f; Extended Data Fig. 15). Furthermore, high-resolution computed tomography (HRCT) images were assessed $24,48,49$ to analyze the association of Ifnarl SNP rs2257167 with clinical features of active TB. TB patients carrying the Ifnarl rs2257167 G allele showed significantly higher HRCT scores (suggesting much severer disease) (Fig. 7g) and less favorable disease outcomes following a WHO-recommended 2HRZE/4HR treatment regimen (Fig. 7h) than patients carrying genotype CC. Collectively, these data indicated that Ifnarl rs2257167 G allele was associated with reduced abundance of A. muciniphila and hyper-reactive TNF- $\alpha$, which led to an increased risk of developing active TB and much severer TB disease in humans.

\subsection{A. muciniphila uses palmitoleic acid to reduce H3K4 tri-methylation (Me3) at} tnfa promoter and inhibits $M$. tuberculosis-specific TNF- $\alpha$ production.

We previously found that histone modification states at tnf $\alpha$ loci determined TNF- $\alpha$ 
production by $\mathrm{T}$ cells ${ }^{50}$. Therefore, we examined whether $A$. muciniphila and $A$. muciniphila-derived palmitoleic acid had any effect on M. tuberculosis-specific TNF$\alpha$ expression by mediating histone modification of $t$ f $f \alpha$ promoter. Immunoblot and ChIP-qPCR analysis showed A. muciniphila indeed induced less enrichment of monomethylation (Me1) of $\mathrm{H} 3 \mathrm{~K} 9$ and tri-methylation (Me3) of $\mathrm{H} 3 \mathrm{~K} 4$ at tnfa promoter regions (Extended Data Fig. 16a-f, 17a-b). Consistently, palmitoleic acid also significantly decreased $M$. tuberculosis-specific TNF- $\alpha$ production in culture supernatants of mouse $\mathrm{T}$ cells, reduced in vivo expression of intracellular TNF- $\alpha$ in expression.

\section{Discussions}

The exact contributions and underlying mechanisms of host genetics, environmental factors, and diet to the structure and function of gut microbiota are still unclear despite recent research advances ${ }^{3-7,11,13,15}$. The precise mechanisms of TB pathogenesis also remain elusive as TB continues to pose as a leading global public health threat ${ }^{16}$. While this study and other groups have shown that the disruption of 
the microbiota contributes to increased susceptibility to TB 29,52 , fecal transplantation $^{29}$ or oral administration of gut bacteria such as Lactobacillus plantarum ${ }^{53}$ has shown the possibility to restore anti-TB immunity or prevent extrapulmonary dissemination of pulmonary TB. However, how human genetics modulate compositional shifts of gut microbiome and gut microbial metabolites and elicit unclear. mechanisms of how IFNAR1 (i.e. IFN-I) signaling modulates the intestinal 
the primary innate defense mechanism to directly or indirectly facilitate the control of gut pathogens ${ }^{44}$, IFN-I signaling driven by a protective ifnarl allele may help establish a favorable gene network or ecosystem for colonization of selected gut bacteria, which may lead to increased growth, colonization, and abundance of $A$. muciniphila. Moreover, since gut bacteria might utilize soluble IgA antibody for colonization in the intestinal mucosa ${ }^{10}$, the gut IFN-I signaling could affect other immune mediators like $\operatorname{IgA}$ antibody and further influence the intestinal colonization of A. muciniphila.

TNF- $\alpha$ is essential to activate innate immunity program for killing intracellular $M$. tuberculosis and is required for TB control ${ }^{59,60}$. Indeed, anti-TNF- $\alpha$ therapies have been shown to be associated with an increased risk of developing active $\mathrm{TB}{ }^{59,61}$. However, hyper-reactive TNF- $\alpha$ inflammation may also be detrimental ${ }^{22,24}$ and the delicate modulation of moderate TNF- $\alpha$ inflammation levels was therefore one of the central ideas for avoiding development of active TB disease. While highly excessive or inappropriate TNF- $\alpha$ production was induced by host genetics-dictated lower abundance of $A$. muciniphila in the intestinal compartment in severe TB diseases, colonization dominance of $A$. muciniphila might help prevent aberration of microbial ecosystem and avoid impairment of homeostasis of immune networks.

$$
\text { In addition, this work demonstrated that host genetics can determine the structure }
$$
and function of gut bacteria and impact the concentration levels and immune regulatory functions of gut bacteria-produced effector metabolites. We have shown that A. muciniphila utilized its effector metabolite, palmitoleic acid, to regulate 

microbiota and its metabolites.

systemic M. tuberculosis-specific TNF- $\alpha$ expression. It would also be interesting to explore whether and what metabolites derived from gut bacteria may migrate into the pulmonary compartment and regulate local inflammation (e.g. local TNF- $\alpha / \mathrm{IL}-1 \beta$ response in or adjacent lung granuloma ${ }^{24}$ ) during $M$. tuberculosis infection. It is worth to explore whether manipulating other host genetic factors and/or other gut bacteria or metabolic pathways capable of producing palmitoleic acid could also confer similar or better anti-TB protection effects. Notably, although butyrate appeared to inhibit TNF- $\alpha$ expression in some disease models or biological settings ${ }^{62-}$ ${ }^{64}$, we did not observe significant inhibition effect on TNF- $\alpha$ expression and anti-TB protective effects of butyrate in this work.

In summary, this study not only demonstrates the previously unrecognized significance of host genetics-gut microbiota interaction axis in regulating varying TB susceptibility and severity but also provides a new paradigm for deciphering the role of gut microbiota in human health and diseases as well as for developing genotypes-dictated precision therapeutics of $\mathrm{TB}$, particularly MDR-TB, targeting gut 


\section{Methods}

Study subjects. This project aims to identify genetic, microbial, and microbial metabolites responsible for the initiation and severity of tuberculosis (TB). This study uses two independent Chinese Han population cohort for 16S rDNA sequencing analysis: the Shenzhen cohort consists of 28 healthy controls (HC) (age range $23.46-$ 54.65 years) and 26 TB patients (age range 23.80 - 47.96 years); the Foshan cohort consists $17 \mathrm{HC}$ (age range 23.36 - 43.84 years) and 19 TB patients (age range 21.85 48.78 years). On entry to the study, subjects are screened using a standardized questionnaire by general practitioner and the demographic information was recorded. Subjects were excluded if they had received probiotics treatment three months before their recruitment into the study. Detailed information on the distributions of age, sex, height, weight, and BMI for each cohort samples are provided in Supplementary

\section{Table 1 and Extended Data Fig. 9a.}

For SNP analysis, this work uses three independent Chinese Han population cohort: Guangzhou cohort as a discovery cohort and Shenzhen cohort and Foshan cohort as replication cohorts. The Guangzhou cohort consists $263 \mathrm{HC}$ (age range $20.78-45.18$ years) and 264 TB patients (age range 19.91 - 55.51 years). The replication cohorts comprise 2978 individuals from Shenzhen cohort (1445 HC, age range $17.00-56.75$ years; 1533 TB patients, age range 21.38 - 52.69 years) and 3007 individuals from Foshan cohort (1679 HC, age range 19.90 - 46.70 years; 1328 TB patients, age range $21.21-49.08$ years). These individuals are not related, neither from a same village or a same family. Detailed information on the distributions of age, sex, height, weight, 
and BMI for all cohort samples are provided in Supplementary Table 2 and

\section{Extended Data Fig. 9b.}

All the patients were prospectively recruited on the basis of TB symptomatic features. The diagnosis of TB was based on clinical signs and symptoms, chest radiography, acid-fast bacilli (AFB) identification (sputum smear, M. tuberculosis culture or nucleic acid amplification techniques (NAAT) assay), and response to antiTB treatment. TB patients with cancers, diabetes, hypertension, or HIV infection were excluded. HC were defined as individuals with normal chest radiograph findings, negative IFN- $\gamma$ release assay (IGRA), no clinical history of TB, other infectious diseases, and major conditions like cancers, diabetes, and hypertension. The study is designed to recruit the $\mathrm{HC}$ in the same regions at the same time. No participant in the cohorts dropped out during the study. All participants were given written informed consent according to protocols approved by the Internal Review and the Ethics Boards of Zhongshan School of Medicine of Sun Yat-sen University.

Isolation of peripheral blood mononuclear cells (PBMCs). PBMCs were isolated from freshly collected heparin lithium blood by Ficoll-Paque Plus density gradient centrifugation. Briefly, Ficoll-Paque Plus was loaded into the blood and centrifuged at $1500 \mathrm{rpm}$ for 20 minutes at $20^{\circ} \mathrm{C}$. The isolated monocytes were washed with $\mathrm{pH} 7.4$ PBS (GIBCO), and finally resuspended in 10\% fetal bovine serum (FBS) / RPMI1640 (GIBCO) medium and cultured for further research.

Cytometric Bead Array (CBA) analysis. Culture supernatants of PBMCs derived 
from $\mathrm{HC}$ and $\mathrm{TB}$, or serum isolated from the M. tuberculosis-infected mice were subject for analysis of the following cytokines: IL-2, IL-4, IL-6, IL-10, TNF- $\alpha$, IFN- $\gamma$, or IL-17A using the BD Cytometric Bead Array (CBA) Th1/Th2 Cytokine Kit or Th1/Th2/Th17 Cytokine Kit (purchased from BD) as previously described ${ }^{50}$. Data were collected on the Beckman CytoFLEX S (Beckman) and analyzed as previously described ${ }^{50}$.

Intracellular cytokine staining (ICS). These experiments were performed as previously described ${ }^{50}$. Cells were first stained with cell-surface markers, and then permeabilized for 30 minutes (cytofix/cytoperm, BD) and stained 45 minutes for intracellular molecules such as TNF- $\alpha$, before being fixated with $2 \%$ formalin/PBS. Cells were then analyzed using polychromatic flow cytometry. Isotype matched IgG staining or blank staining served as negative controls to ensure the specificity of ICS experiments. Data were collected on the Beckman CytoFLEX S (Beckman) and analyzed with Kaluza 1.2 software (Beckman).

Antibodies. Rabbit-signal transducer and activator of transcription 1 (STAT1) (sc346), rabbit-phosphorylated- (p-) STAT1 (\#9167,CST), rabbit anti H3K9Me1 (ab9045, Abcam), mouse anti-H3K27Me3 (ab6002, Abcam), rabbit anti-H3K4Me3 (ab8580, Abcam), rabbit anti-H3 (ab1791, Abcam), rabbit anti- $\beta$ actin (ab6276, Abcam), rabbit anti-H3K27Me2 (ab24684, Abcam), mouse anti-H3k9Me2 (ab1220, Abcam), rabbit anti-H3K9Me3 (ab8898, Abcam), CD4-APC-CY7 (GK1. 5, BioLegend), CD8-V450 (53-6.7, BioLegend), TNF- $\alpha$ (MP6-XT22, BioLegend), anti- 
fecal samples). Fresh fecal samples were collected from TB and HC. DNA was

459 isolated with the DNA Stools Kit (QIAGEN) by following the manufacturer's 460 instructions. Garose gel electrophoresis detection was performed for the purity and concentrations of DNA in fecal samples from the Shenzhen cohort. The corresponding primers were used to amplify DNA: $341 \mathrm{~F}$ and $806 \mathrm{R}$ for $\mathrm{V} 3+\mathrm{V} 4$ regions, $515 \mathrm{~F}$ and $907 \mathrm{R}$ for $\mathrm{V} 4+\mathrm{V} 5$ regions, $515 \mathrm{~F}$ and $806 \mathrm{R}$ for $\mathrm{V} 4$ regions. Amplified DNA fragments were PE250 paired-end sequenced by Illumina NovaSeq. For DNA of fecal samples derived from the Foshan cohort, the corresponding primers were used to amplify DNA: 341F-805R for V3+V4 regions, 338F-533R for the V3 Regions, and 967F-1046R for the V6 regions. The index sequences were added and

RAD CFX 96 were used to quantify the concentrations and purity of the library to ensure DNA quality. Afterward, the library was sequenced on an Illumina HiSeq2500 using the 250 paired-end protocol.

Antibiotics treatment. A mixture of ampicillin $(1 \mathrm{mg} / \mathrm{ml})$, streptomycin $(5 \mathrm{mg} / \mathrm{ml})$, and colistin $(1 \mathrm{mg} / \mathrm{ml})$ (Sigma-Aldrich) was added to sterile drinking water for mice. Solutions and bottles were changed 3 times per week. Antibiotic activity was confirmed by cultivating fecal pellets resuspended in BHI $+15 \%$ glycerol at $0.1 \mathrm{~g} / \mathrm{ml}$

476 and cultured on blood agar plates for 48 hours at $37^{\circ} \mathrm{C}$ in aerobic and anaerobic 
conditions weekly.

Gut colonization with commensal species. A. muciniphila (ATCC BAA-835) and B.vulgatus (ATCC-8482) were purchased from the ATCC and grown on COS (Columbia Agar with 5\% Sheep Blood) plates in an anaerobic atmosphere at $37^{\circ} \mathrm{C}$ for at least 72 hours and 48 hours, respectively. Bacteria were harvested from the agar plates and suspended in sterile saline with $10 \%$ glycerol to obtain suspensions of $10^{9}$ colony-forming unit $(\mathrm{CFU}) / \mathrm{ml}$ at an optical density of $600 \mathrm{~nm}$. Gut colonization of antibiotics pre-treated mice was performed by oral gavage with $200 \mu 1$ suspension containing $2 \times 10^{8}$ bacteria for 3 times per week.

Dietary treatment of metabolites. Palmitoleic acid (Sigma) or butyrate (Sigma) was added to drinking water containing $\mathrm{NaOH}$ at a final concentration of $36 \mathrm{mM}^{65}$. Water with same concentration of $\mathrm{NaOH}$ was used as control. $100 \mathrm{mg}$ palmitoleic acid was dissolved in $0.1 \mathrm{M} \mathrm{NaOH}$ aqueous solution.

Mice and infection. Specific-pathogen free C57BL/6 mice were purchased from Sun Yat-sen University Laboratory Animal Center. Transgenic mice were constructed using microinjection technology for DNA fragments consisting of the human Ifnarl gene "G" allele or "C" allele to randomly integrate into the mouse's intestinal epitheliums genome in the C57BL/6 background (Extended Data Fig. 12a). For $M$. tuberculosis infection, each mouse was infected through aerosols with approximately $150 \mathrm{CFU}$ M. tuberculosis (H37Rv) for five weeks at Biosafety Level-3 (BSL-3) Laboratory of Sun Yat-sen University (SYSU). 
Bacterial and histopathological analysis of $M$. tuberculosis-infected mice. To determine M. tuberculosis burden, lungs of mice were homogenized carefully for $M$. tuberculosis CFU counting analysis as we previously described ${ }^{50}$. For tissue histopathological analysis, lung tissues of mice were fixed in $10 \%$ zinc formalin and embedded in paraffin. Five $\mu$ m-thick sections were stained with hematoxylin and eosin (H\&E) and images were obtained using Digital Slide Scanning System AxioScan Z1. Images of acid-fast staining were obtained under a microscope (Olympus BX51). An overall histology score was assigned to the lungs of mice based on the degree of granulomatous inflammation as follows: $0=$ no lesion, $1=$ minimal lesion $(1-10 \%$ area of tissue in the section involved), $2=$ mild lesion $(11-30 \%$ area involved), $3=$ moderate lesion $(30-50 \%$ area involved), $4=$ marked lesion $(50-80 \%$ area involved), and $5=$ severe lesion $(>80 \%$ area involved).

Transwell assays. To mimic the effects of epithelium-colonized gut bacteria on cytokine production, a transwell assay was performed using 24-well transwell plates with $0.4 \mu \mathrm{m}$-pore inserts (Corning). $2 \times 10^{5}$ intestinal epitheliums in RPMI 1640 medium (Gibco) with 10\% FBS with $1 \times 10^{5} \mathrm{CFU}$ A. muciniphila or medium were seeded into the upper chamber, while $2 \times 10^{5} \mathrm{CD} 3+\mathrm{T}$ lymphocytes isolated from the spleens of autologous mouse in RPMI 1640 (Gibco) with 10\% FBS were added to the lower chamber. $\mathrm{T}$ cell isolation was performed as previously described ${ }^{50}$. After 12 hours of incubation, the productions of cytokines in the lower chamber were detected by CBA analysis. 

scoring. HRCT was performed at $10 \mathrm{~mm}$-section interval $(120 \mathrm{kV}, 50-450 \mathrm{mAs}, 1$

$521 \mathrm{~mm}$ slice thickness, 1.5 second scanning time) with a window level between 2550 and 40 Hounsfield Units (HU) and window width between 300 and $1600 \mathrm{HU}$ using the Toshiba Aquilion 64 CT Scanner (Toshiba, Tokyo, Japan). HRCT scans were analyzed by two independent chest radiologists and conclusions on the findings were reached by consensus. Radio-pathological changes were quantified using a scoring system developed by Ors et al. The arbitrary scores were based on the percentage of lung parenchyma abnormality as we and others previously described ${ }^{48,49}$.

DNA extraction and SNP genotyping. Fresh blood samples were collected from TB and HC. Genomic DNA was isolated using the DNA Blood Kit (QIAGEN) following the manufacturer's instructions. In the TB discovery study, we used targeted sequencing of the 11 exons in human Ifnarl gene and found a SNP rs2257167 in the fourth exon in HC and TB. For validation cohorts, SNP rs2257167 was genotyped in HC and TB using TaqMan assays (ABI, Carlsbad, CA).

Western Blots and chemical reagents. Cells were isolated from indicated individuals or mice and were treated differently based on specific experimental settings. Cells were co-cultured with Sendai virus in some experiments. Total proteins were extracted from cells using RIPA lysis buffer and quantified using BCA Protein Assay Kit (Thermo Fisher Scientific). Extracted proteins mixed with loading buffer were electrophoresed through 10\% SDS-PAGE and then transferred to PVDF 
membranes (Bio-Rad), which were then blocked in 5\% fat-free milk for one hour. The membranes were incubated with primary antibodies at $4{ }^{\circ} \mathrm{C}$ overnight, and further incubated with a secondary antibody (Thermo Fisher) for 1 hour at room temperature. Signals were detected using Immobilon ${ }^{\mathrm{TM}}$ Western Chemiluminescent HRP Substrate (Millipore). Images were captured by the BIO-RAD ChemiDoc Touch machine and analyzed by ImageJ 1.43 software.

Chromatin Immunoprecipitation (ChIP) and ChIP-qPCR. CD3 $+\mathrm{T}$ cells were isolated from spleens of mice and treated with M. tuberculosis and A. muciniphila lysates or metabolites. $1 \times 10^{6}$ cells were collected for each round of ChIP. ChIP was performed using ChIP Assay Kit (Millipore) according to the manufacturer's manual. The antibodies used included rabbit anti-H3K9Me1 (ab9045, Abcam), mouse antiH3K27Me3 (ab6002, Abcam), rabbit anti-H3K4Me3 (ab8580, Abcam), rabbit anti-H3 (ab1791, Abcam), rabbit anti-H3K27Me2 (ab24684, Abcam), mouse anti-H3k9Me2 (ab1220, Abcam), and rabbit anti-H3K9Me3 (ab8898, Abcam). Real-time qPCR was performed with $2 \mu \mathrm{l}$ of the immunoprecipitated DNA.

Detection of anaerobic bacteria and $A$. muciniphila. The jejunum, cecum, ileum, and fecal samples of each mouse were homogenized in PBS, respectively. Ten-fold serial dilutions were made using PBS and plated on COS plates (Columbia Agar with $5 \%$ Sheep Blood), and incubated at $37^{\circ} \mathrm{C}$ in anaerobic conditions for 48 hours or at least 72 hours based on experiment settings. Genomic DNA was isolated from tissues or fecal samples using the QIAamp DNA Tissue or Stools Mini Kit (Qiagen) 
561

562

563

following the manufacturer's instructions. Targeted qPCR systems were applied using either TaqMan or SYBR Green technology. The primers and probes were used as previously described ${ }^{66}$.

Liquid culture of $A$. muciniphil, B.vulgatus and L. salivarius. Cultures of $A$. muciniphila (ATCC BAA-835) were grown in brain heart infusion (BHI) for at least 72 hours at $37^{\circ} \mathrm{C}$ in anaerobic conditions. Cultures of B.vulgatus (ATCC-8482), which were purchased from the ATCC, were grown in BHI for at least 48 hours at $37^{\circ} \mathrm{C}$ in anaerobic conditions. Cultures of L. salivarius (BNCC 138618) were grown in Man Rogosa Sharpe (MRS) for at least 48 hours at $37^{\circ} \mathrm{C}$ in anaerobic conditions.

Liquid culture of $\boldsymbol{A}$. johnsonii. Cultures of $A$. johnsonii (BNCC 341940) were grown in Zobell Marine Broth 2216 (2216E) for at least 48 hours at $30^{\circ} \mathrm{C}$ in aerobic conditions.

\section{Bacterial cell ingredients, bacterial culture supernatant, and plasma metabolites}

extraction. $100 \mu \mathrm{l}$ samples were added into $2 \mathrm{ml} \mathrm{EP}$ tubes. $0.35 \mathrm{ml}$ extraction liquid (VMethanol) containing $20 \mu \mathrm{l}$ of L-2-Chlorophenylalanine $(1 \mathrm{mg} / \mathrm{ml}$ stock in $\mathrm{dH} 2 \mathrm{O}$ ) was added to each sample as an internal standard. Samples were vortexed for 30 seconds and then centrifuged for 15 minutes at $4^{\circ} \mathrm{C}, 12000 \mathrm{rpm}$. Supernatants $(0.4 \mathrm{ml})$ were transferred into a fresh $2 \mathrm{ml} \mathrm{GC/MS}$ glass vial and then spend-vacuumed to dry. $40 \mu \mathrm{l}$ Methoxy amination hydrochloride $(20 \mathrm{mg} / \mathrm{ml}$ in pyridine) was added to each sample and incubated for 30 minutes at $80^{\circ} \mathrm{C}$. Then, $60 \mu \mathrm{l}$ of the BSTFA regent $(1 \%$ TMCS, v/v) was added to the sample aliquots and incubated for 1.5 hours at $70^{\circ} \mathrm{C}$. All 
582

samples were analyzed by a gas chromatograph system coupled with a Pegasus HT time-of-flight mass spectrometer (GC-TOF-MS).

Fecal metabolite extraction. $100 \pm 1 \mathrm{mg}$ samples were added into $2 \mathrm{ml} \mathrm{EP}$ tubes. $0.48 \mathrm{ml}$ of extraction liquid (VMethanol: VChloroform $=3: 1$ ) containing $20 \mu \mathrm{l}$ of L2-Chlorophenylalanine $(1 \mathrm{mg} / \mathrm{ml}$ stock in $\mathrm{dH} 2 \mathrm{O})$ was added to each sample as internal standard followed by vortex mixing for 30 seconds. Samples were homogenized in ball mill for 4 minutes at $45 \mathrm{~Hz}$, then treated with ultrasound for 5 minutes (incubated in ice water) for 5 times and centrifuged for 15 minutes at $4^{\circ} \mathrm{C}, 12000 \mathrm{rpm}$. The supernatant $(0.4 \mathrm{ml})$ was transferred into a fresh $2 \mathrm{ml} \mathrm{GC} / \mathrm{MS}$ glass vial and then spend-vacuumed to dry. $40 \mu \mathrm{l}$ Methoxy amination hydrochloride $(20 \mathrm{mg} / \mathrm{ml}$ in pyridine) was added to each sample and incubated for 30 minutes at $80^{\circ} \mathrm{C}$. Then, $60 \mu 1$ of the BSTFA regent ( $1 \%$ TMCS, v/v) was added to the sample aliquots and incubated for 1.5 hours at $70^{\circ} \mathrm{C}$. All samples were analyzed by a gas chromatograph system coupled with a Pegasus HT time-of-flight mass spectrometer (GC-TOF-MS).

GC-TOF-MS Analysis. All GC-TOF-MS analyses were performed using an Agilent 7890 gas chromatograph system coupled with a Pegasus HT time-of-flight mass spectrometer. The system utilized a DB-5MS capillary column coated with $5 \%$ diphenyl cross-linked with $95 \%$ dimethylpolysiloxane $(30 \mathrm{~m} \times 250 \mu \mathrm{m}$ inner diameter, $0.25 \mu \mathrm{m}$ film thickness; J \& W Scientific, Folsom, CA, USA). A $1 \mu$ aliquot of the analyte was injected in splitless mode. Helium was used as the carrier gas; the front inlet purge flow was set at $3 \mathrm{ml} / \mathrm{min}$, and the gas flow rate through the column was 1 
603

604

605

606

607

608

609

610

611

612

613

614

615

616

617

618

619

620

621

622

623

$\mathrm{ml} / \mathrm{min}$. The initial temperature was kept at $50^{\circ} \mathrm{C}$ for 1 minute, then raised to $310^{\circ} \mathrm{C}$ at a rate of $10^{\circ} \mathrm{C} / \mathrm{min}$, and kept at $310^{\circ} \mathrm{C}$ for 8 minutes. The injection, transfer line, and ion source temperatures were $280^{\circ} \mathrm{C}, 280^{\circ} \mathrm{C}$, and $250^{\circ} \mathrm{C}$, respectively. The energy was $-70 \mathrm{eV}$ in electron impact mode. The mass spectrometry data were acquired in fullscan mode with the $\mathrm{m} / \mathrm{z}$ range of $50-500$ at a rate of 20 spectra per second after a solvent delay of $6.27 \mathrm{~min}$.

Analysis of GC-TOF-MS. Data were collected using the Chroma TOF 4.3X software (LECO Corporation) and the LECO-Fiehn Rtx5 database were used for raw peaks exacting, data baselines filtering and calibration of the baseline, peak alignment, deconvolution analysis, peak identification, and integration of the peak area ${ }^{67}$. Both mass spectrum match and retention index match were considered in metabolites identification.

RNA Extraction and Real-Time PCR. Total RNA was extracted from tissues or PBMCs using Trizol reagent (Takara, Japan) and total RNA concentration was determined by a Nanodrop spectrometer. First-strand cDNA was synthesized using the PrimeScript One Step Strand cDNA Synthesis Kit (Takara, Japan) following the manufacturer's instructions; qPCR was performed in technical triplicates using SYBR Green to determine the expression of $I F N-\beta$ and $I S G 15$. GADPH was used as an endogenous control to normalize gene expression (Supplementary Table 5). Relative mRNA expression levels were presented as means \pm SEM. Statistical differences were analyzed by Student's $t$-test. 
The jejunum, cecum, and ileum from mono-colonized mice were trimmed into 2-3 mm cube size and immediately fixed with an ice-cold solution of $3 \%$ glutaraldehyde in $0.1 \mathrm{M}$ sodium cacodylate buffer at $4^{\circ} \mathrm{C}$ overnight. Samples were then post-fixed in $2 \%$ osmium tetroxide buffered solution and were embedded in epoxy resin. Subsequently, samples were processed as previously described ${ }^{10}$. Micrographs were produced using a FEI Tecnai G2 Spirit BioTwin 634 transmission electron microscope.

\section{Fluorescent in situ hybridization (FISH) and immunofluorescence of $A$.} muciniphila in cecum. Paraformaldehyde-fixed, paraffin-embedded colon tissue sections $(5 \mu \mathrm{m})$ were deparaffinized. A specific fluorescein-labeled oligonucleotide probe ${ }^{66}$ targeting one region of the $16 \mathrm{~S}$ rDNA gene of $A$. muciniphila was used to detect bacterial colonization. Monitoring of nonspecific hybridizations was done using the probe Non-EUB ${ }^{68}$ as a negative control. These probes were hybridized to the tissue overnight at $50^{\circ} \mathrm{C}$. Then, slides mounted with ProLong ${ }^{\circledR}$ Gold with DAPI (Invitrogen) were sealed with coverslips, left to dry at $4^{\circ} \mathrm{C}$ in the dark overnight, and imaged using a confocal microscope (LSM 880 with Airyscan).

Statistical analysis. Statistical analysis was performed using GraphPad Prism software. For SNPs and TB susceptibility studies, (1) the Hardy-Weinberg Equilibrium analysis for Ifnarl SNP distribution was used in TB patients and HC; (2)The Pearson $\chi^{2}$ test was used to compare the genotypic and allelic frequencies of 
645 SNPs between patients and controls; (3) Unconditional logistic regression adjusted by 646 gender and age was used to calculate the odd ratios (OR), 95\% confidence intervals 647 (CIs), and corresponding $p$ values using four alternative models (multiplicative, 648 additive, dominant and recessive). For statistical analysis of other experiments, 649 Normal distributions of data was determined with D'Agostino-Pearson omnibus test, 650 statistical significance was determined using one-way ANOVA with Newman-Keuls 651 or Tukey's multiple comparison test, Student's two-tailed unpaired $t$-test, or Mann652 Whitney U test. $P<0.05$ was considered as statistically significant. ${ }^{*} P<0.05$, ${ }^{*} P<$ $6530.01, * * * P<0.001$, and $* * * * P<0.0001$. NS, no statistical significance. Odds ratio 654 and $P$-value for SNP analysis were determined by SPSS. 
657 Data of 16S rDNA sequencing are available at https://dataview.ncbi.nlm.nih.gov/. 658 The accession number of $16 \mathrm{~S}$ rDNA sequencing data is PRJNA609532. Fig. 1, 659 Extended Data Fig. 1, and Extended Data Fig. 2 have associated raw data. All other 660 data are available from the corresponding author upon reasonable request.

661 
662

663

664

665

666

667

668

669

670

671

672

673

674

675

676

677

680

\section{Acknowledgments}

This work was supported by NSFC Grants 82072250 (To G.C.Z.), and 81873958 (To G.L.Z); National Key R\&D Plan Grant 2016YFE0106900 (To G.C.Z.); The National Science and Technology Major Project 2017ZX10201301 and 2017ZX106019, and $2017 Z X 10103004$ (To G.L.Z.); Guangdong Science and Technology Project Grant 2018A050506032 (To G.C.Z.); Shenzhen Scientific and Technological Foundation JCYJ20180228162511084 (To G.L.Z.); and Sanming Project of Medicine in Shenzhen SZSM201911009 (To G.L.Z.).

\section{Author Contributions}

L.M.C., Z.H.G., Y.Y., Z.Y.Z., Y.W.C., Q.D.M., Y.W., S.Y., G.H.Z., J.P., L.C.Z. and G.B.L. performed the experiments and analyzed the data. G.L.Z., G.B.L., Z.L., W.W., X.H., and L.Z. collected clinical samples and conducted analysis. S.Y. and J.C. helped with signaling analysis experiments. X.H. and G.L.Z. performed SNP and clinical analysis works, and X.H., Z.L., J.C., L.S., L.C.Z., H.P.L., X.C.C., and B.X.G. assisted data analysis and manuscript preparations. L.M.C., G.C.Z., Z.W.C., X.C.C., and B.X.G. drafted, discussed, and revised the manuscript. G.C.Z. conceived this study.

\section{Declaration of Competing Interests None}




\section{References}

682 1. Schirmer, M., et al. Dynamics of metatranscription in the inflammatory bowel disease gut microbiome. Nat Microbiol 3, 337-346 (2018). Fujimura, K.E. \& Lynch, S.V. Microbiota in allergy and asthma and the emerging relationship with the gut microbiome. Cell Host Microbe 17, 592-602 (2015).

3. Rothschild, D., et al. Environment dominates over host genetics in shaping human gut

4. David, L.A., et al. Diet rapidly and reproducibly alters the human gut microbiome. Nature 505,

5. Wu, G.D., et al. Linking long-term dietary patterns with gut microbial enterotypes. Science 334, 105-108 (2011).

6. Muegge, B.D., et al. Diet drives convergence in gut microbiome functions across mammalian phylogeny and within humans. Science 332, 970-974 (2011).

7. Ley, R.E., Turnbaugh, P.J., Klein, S. \& Gordon, J.I. Microbial ecology: human gut microbes associated with obesity. Nature 444, 1022-1023 (2006).

8. Kawamoto, S., et al. The inhibitory receptor PD-1 regulates IgA selection and bacterial composition in the gut. Science 336, 485-489 (2012).

9. Hapfelmeier, S., et al. Reversible microbial colonization of germ-free mice reveals the dynamics of IgA immune responses. Science 328, 1705-1709 (2010).

10. Donaldson, G.P., et al. Gut microbiota utilize immunoglobulin A for mucosal colonization. Science 360, 795-800 (2018).

11. Bonder, M.J., et al. The effect of host genetics on the gut microbiome. Nat Genet 48, 14071412 (2016).

12. Igartua, C., et al. Host genetic variation in mucosal immunity pathways influences the upper airway microbiome. Microbiome 5, 16 (2017).

13. Goodrich, J.K., et al. Human genetics shape the gut microbiome. Cell 159, 789-799 (2014).

14. Lim, M.Y., et al. The effect of heritability and host genetics on the gut microbiota and metabolic syndrome. Gut 66, 1031-1038 (2017).

15. Wang, J., et al. Genome-wide association analysis identifies variation in vitamin $D$ receptor and other host factors influencing the gut microbiota. Nat Genet 48, 1396-1406 (2016).

16. WHO. Global tuberculosis report. Geneva: World Health Organization (2019).

17. Bradley, C.P., et al. Segmented Filamentous Bacteria Provoke Lung Autoimmunity by Inducing Gut-Lung Axis Th17 Cells Expressing Dual TCRs. Cell Host Microbe 22, 697-704 e694 (2017).

18. Gauguet, S., et al. Intestinal Microbiota of Mice Influences Resistance to Staphylococcus aureus Pneumonia. Infect Immun 83, 4003-4014 (2015).

19. Schuijt, T.J., et al. The gut microbiota plays a protective role in the host defence against pneumococcal pneumonia. Gut 65, 575-583 (2016).

20. Budden, K.F., et al. Emerging pathogenic links between microbiota and the gut-lung axis. Nat Rev Microbiol 15, 55-63 (2017).

21. Wypych, T.P., Wickramasinghe, L.C. \& Marsland, B.J. The influence of the microbiome on respiratory health. Nat Immunol 20, 1279-1290 (2019). 
22. Roca, F.J. \& Ramakrishnan, L. TNF dually mediates resistance and susceptibility to mycobacteria via mitochondrial reactive oxygen species. Cell 153, 521-534 (2013).

23. Stallings, C.L. Host response: Inflammation promotes TB growth. Nat Microbiol 2, 17102 (2017).

24. Mishra, B.B., et al. Nitric oxide prevents a pathogen-permissive granulocytic inflammation during tuberculosis. Nat Microbiol 2, 17072 (2017).

25. Zumla, A., et al. Host-directed therapies for infectious diseases: current status, recent progress, and future prospects. Lancet Infect Dis 16, e47-63 (2016).

26. Orme, I.M., Robinson, R.T. \& Cooper, A.M. The balance between protective and pathogenic immune responses in the TB-infected lung. Nat Immunol 16, 57-63 (2015).

27. Botero, L.E., et al. Respiratory tract clinical sample selection for microbiota analysis in patients with pulmonary tuberculosis. Microbiome 2, 29 (2014).

28. Namasivayam, S., et al. Longitudinal profiling reveals a persistent intestinal dysbiosis triggered by conventional anti-tuberculosis therapy. Microbiome 5, 71 (2017).

29. Khan, N., et al. Alteration in the Gut Microbiota Provokes Susceptibility to Tuberculosis. Front Immunol 7, 529 (2016).

30. Derrien, M., Vaughan, E.E., Plugge, C.M. \& de Vos, W.M. Akkermansia muciniphila gen. nov., sp. nov., a human intestinal mucin-degrading bacterium. Int J Syst Evol Microbiol 54, 14691476 (2004).

31. Miron B. Kursa, W.R.R. Feature Selection with the Boruta Package. Journal of Statistical Software 36(11), p. 1-13 (2010).

32. Hu, Y., et al. The Gut Microbiome Signatures Discriminate Healthy From Pulmonary Tuberculosis Patients. Front Cell Infect Microbiol 9, 90 (2019).

33. Behar, S.M. \& Sassetti, C.M. Immunology: Fixing the odds against tuberculosis. Nature 511, 39-40 (2014).

34. Ernst, J.D. The immunological life cycle of tuberculosis. Nat Rev Immunol 12, 581-591 (2012).

35. Flynn, J.L. \& Chan, J. Immunology of tuberculosis. Annu Rev Immunol 19, 93-129 (2001).

36. Kaufmann, S.H.E., Dorhoi, A., Hotchkiss, R.S. \& Bartenschlager, R. Host-directed therapies for bacterial and viral infections. Nat Rev Drug Discov 17, 35-56 (2018).

37. O'Garra, A., et al. The immune response in tuberculosis. Annu Rev Immunol 31, 475-527 (2013).

38. Zeng, G., Zhang, G. \& Chen, X. Th1 cytokines, true functional signatures for protective immunity against TB? Cell Mol Immunol 15, 206-215 (2018).

39. Wang, L., et al. Oxidization of TGFbeta-activated kinase by MPT53 is required for immunity to Mycobacterium tuberculosis. Nat Microbiol 4, 1378-1388 (2019).

40. Qiu, L., et al. Severe tuberculosis induces unbalanced up-regulation of gene networks and overexpression of IL-22, MIP-1alpha, CCL27, IP-10, CCR4, CCR5, CXCR3, PD1, PDL2, IL-3, IFNbeta, TIM1, and TLR2 but low antigen-specific cellular responses. J Infect Dis 198, 1514-1519 (2008).

41. Teles, R.M., et al. Type I interferon suppresses type II interferon-triggered human antimycobacterial responses. Science 339, 1448-1453 (2013).

42. Berry, M.P., et al. An interferon-inducible neutrophil-driven blood transcriptional signature in human tuberculosis. Nature 466, 973-977 (2010).

43. Mayer-Barber, K.D., et al. Host-directed therapy of tuberculosis based on interleukin-1 and 
type I interferon crosstalk. Nature 511, 99-103 (2014).

44. Tschurtschenthaler, M., et al. Type I interferon signalling in the intestinal epithelium affects Paneth cells, microbial ecology and epithelial regeneration. Gut 63, 1921-1931 (2014).

45. Sun, L., et al. Type I interferons link viral infection to enhanced epithelial turnover and repair. Cell Host Microbe 17, 85-97 (2015).

46. Zhang, G., et al. A proline deletion in IFNAR1 impairs IFN-signaling and underlies increased resistance to tuberculosis in humans. Nat Commun 9, 85 (2018).

47. Zhang, G., et al. A functional single-nucleotide polymorphism in the promoter of the gene encoding interleukin 6 is associated with susceptibility to tuberculosis. J Infect Dis 205, 16971704 (2012).

48. Zhang, G., et al. Allele-specific induction of IL-1beta expression by C/EBPbeta and PU.1 contributes to increased tuberculosis susceptibility. PLoS Pathog 10, e1004426 (2014).

49. Ors, F., et al. High-resolution CT findings in patients with pulmonary tuberculosis: correlation with the degree of smear positivity. J Thorac Imaging 22, 154-159 (2007).

50. Wang, Y., et al. Long noncoding RNA derived from CD244 signaling epigenetically controls CD8+ T-cell immune responses in tuberculosis infection. Proc Natl Acad Sci U S A 112, E38833892 (2015).

51. Wang, J., et al. Sequence features and chromatin structure around the genomic regions bound by 119 human transcription factors. Genome Res 22, 1798-1812 (2012).

52. Khan, N., et al. Intestinal dysbiosis compromises alveolar macrophage immunity to Mycobacterium tuberculosis. Mucosal Immunol 12, 772-783 (2019).

53. Negi, S., Pahari, S., Bashir, H. \& Agrewala, J.N. Gut Microbiota Regulates Mincle Mediated Activation of Lung Dendritic Cells to Protect Against Mycobacterium tuberculosis. Front Immunol 10, 1142 (2019).

54. Giosue, S., et al. Effects of aerosolized interferon-alpha in patients with pulmonary tuberculosis. Am J Respir Crit Care Med 158, 1156-1162 (1998).

55. Boxx, G.M. \& Cheng, G. The Roles of Type I Interferon in Bacterial Infection. Cell Host Microbe 19, 760-769 (2016).

56. McNab, F., Mayer-Barber, K., Sher, A., Wack, A. \& O'Garra, A. Type I interferons in infectious disease. Nat Rev Immunol 15, 87-103 (2015).

57. Moreira-Teixeira, L., Mayer-Barber, K., Sher, A. \& O'Garra, A. Type I interferons in tuberculosis: Foe and occasionally friend. J Exp Med 215, 1273-1285 (2018).

58. Ji, D.X., et al. Type I interferon-driven susceptibility to Mycobacterium tuberculosis is mediated by IL-1Ra. Nat Microbiol 4, 2128-2135 (2019).

59. Flynn, J.L., et al. Tumor necrosis factor-alpha is required in the protective immune response against Mycobacterium tuberculosis in mice. Immunity 2, 561-572 (1995).

60. Miller, E.A. \& Ernst, J.D. Anti-TNF immunotherapy and tuberculosis reactivation: another mechanism revealed. J Clin Invest 119, 1079-1082 (2009).

61. Keane, J., et al. Tuberculosis associated with infliximab, a tumor necrosis factor alphaneutralizing agent. N Engl J Med 345, 1098-1104 (2001).

62. Aden, K., et al. Metabolic Functions of Gut Microbes Associate With Efficacy of Tumor Necrosis Factor Antagonists in Patients With Inflammatory Bowel Diseases. Gastroenterology 157, 1279-1292 e1211 (2019).

63. Scott, N.A., et al. Antibiotics induce sustained dysregulation of intestinal T cell immunity by 
perturbing macrophage homeostasis. Sci Trans/ Med 10(2018).

64. Lachmandas, E., et al. Diabetes Mellitus and Increased Tuberculosis Susceptibility: The Role of Short-Chain Fatty Acids. J Diabetes Res 2016, 6014631 (2016).

65. Arpaia, N., et al. Metabolites produced by commensal bacteria promote peripheral regulatory T-cell generation. Nature 504, 451-455 (2013).

66. Collado, M.C., Derrien, M., Isolauri, E., de Vos, W.M. \& Salminen, S. Intestinal integrity and Akkermansia muciniphila, a mucin-degrading member of the intestinal microbiota present in infants, adults, and the elderly. Appl Environ Microbiol 73, 7767-7770 (2007).

67. Kind, T., et al. FiehnLib: mass spectral and retention index libraries for metabolomics based on quadrupole and time-of-flight gas chromatography/mass spectrometry. Anal Chem 81, 10038-10048 (2009).

68. Amann, R.l., et al. Combination of $16 \mathrm{~S}$ rRNA-targeted oligonucleotide probes with flow cytometry for analyzing mixed microbial populations. Appl Environ Microbiol 56, 1919-1925 (1990). 
Fig. 1
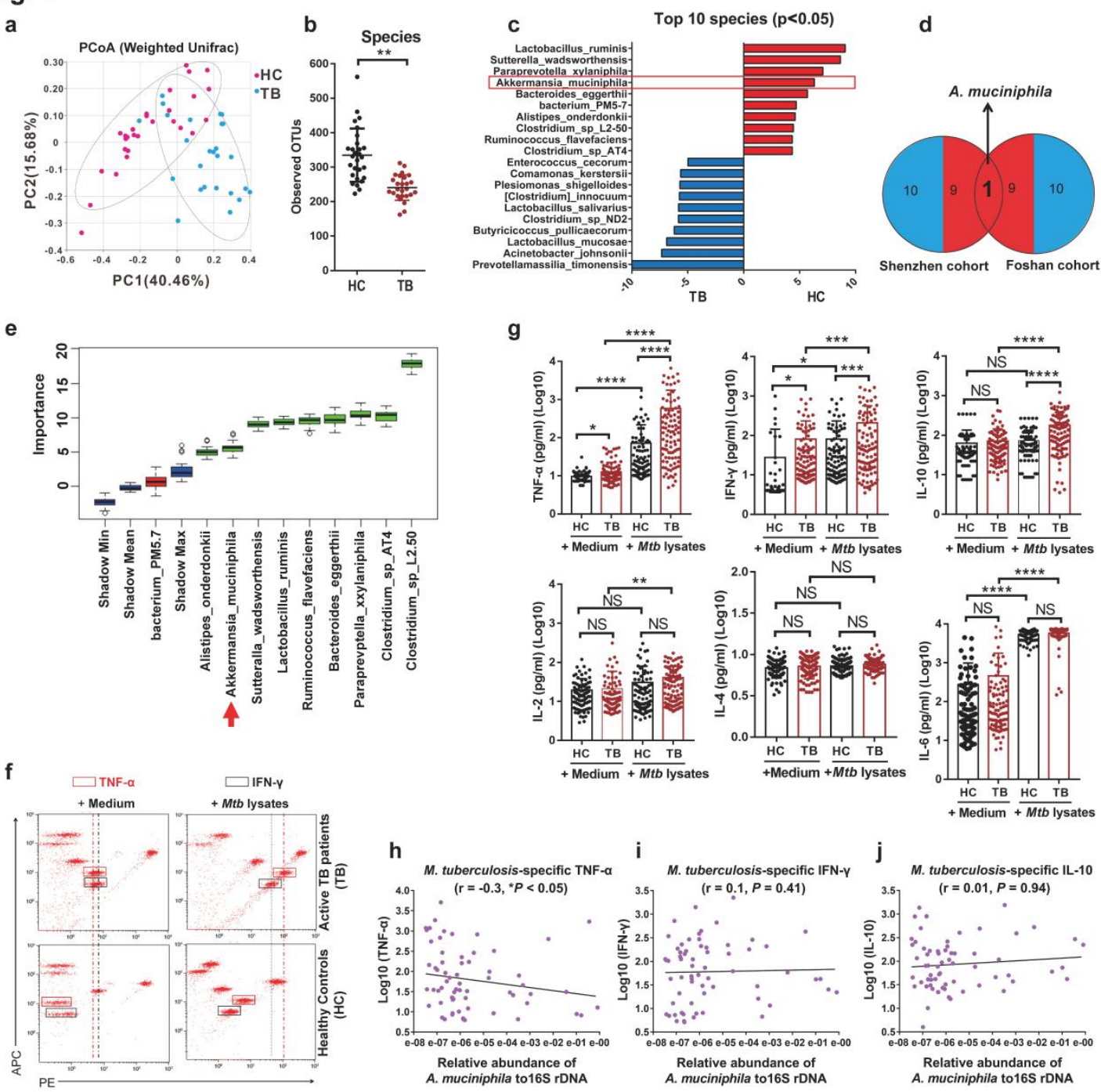

Fig. 1. Reduction of $A$. muciniphila abundance is associated with active TB infection and higher M. tuberculosis-specific TNF- $\alpha$ production in humans.

(a) Principal Co-ordinates Analysis (PCoA) plot (based on weighted UniFrac distances) ( $\mathrm{HC}=28, \mathrm{~TB}=26$, Shenzhen cohort).

(b) Numbers of observed operational taxonomic units (OTUs) of species in fecal microbiota of indicated individuals $(\mathrm{HC}=28, \mathrm{~TB}=26$, Shenzhen cohort $)$.

(c) The top 10 bacterial species with enriched relative abundance in $\mathrm{HC}$ were adjusted $P<0.05$ and $\log 2(\mathrm{HC} / \mathrm{TB})>0$, species with enriched relative abundance in $\mathrm{TB}$ were adjusted $P<0.05$ and $\log 2(\mathrm{HC} / \mathrm{TB})<0(\mathrm{HC}, \mathrm{n}=28 ; \mathrm{TB}, \mathrm{n}=26)$. The red 
box indicates $A$. muciniphila

(d) Venn diagram analysis of top 10 species enriched in $\mathrm{HC}$ or TB from Shenzhen and Foshan cohorts.

(e) Predictive power of top 10 species enriched in HC (i.e. the top 10 most reduced species in TB) assessed by random forest analysis. Blue boxplots acted as benchmarks. Green boxplots represent confirmed species and red boxplots represent rejected species. Red arrowhead marks A. muciniphila.

(f) CBA analysis of culture supernatants of PBMCs derived from TB patients and HC in the Shenzhen cohort. Red- and black-boxed areas mark fluorescent clusters of TNF- $\alpha$ and IFN- $\gamma$, respectively, and dashed lines mark the shift of fluorescent clusters of TNF- $\alpha$ and IFN- $\gamma$, respectively.

(g) Pooled bar data show expressions of TNF- $\alpha /$ IFN- $\gamma /$ IL-2/IL-4/IL-6/IL-10 in cultured supernatants of PBMCs derived from TB $(n=96)$ and HC $(n=96)$ of Shenzhen cohort.

(h-j) Spearman correlation analysis shows the association between ex vivo concentration of TNF- $\alpha / \mathrm{IFN}-\gamma / \mathrm{IL}-10$ and the relative abundance of $A$. muciniphila in fecal samples of TB $(n=63)$.

Error bars indicate SD. $P<0.05\left(^{*}\right) ; P<0.01(* *) ; P<0.001(* * *) ; P<0.0001$ (****); NS (no statistical significance). $P$ values were calculated by Mann-Whitney $U$ test [(b) and (c)], one-way ANOVA with Newman-Keuls multiple comparison test (g), and Spearman correlation $(\mathrm{h}-\mathrm{j})$. 
Fig. 2
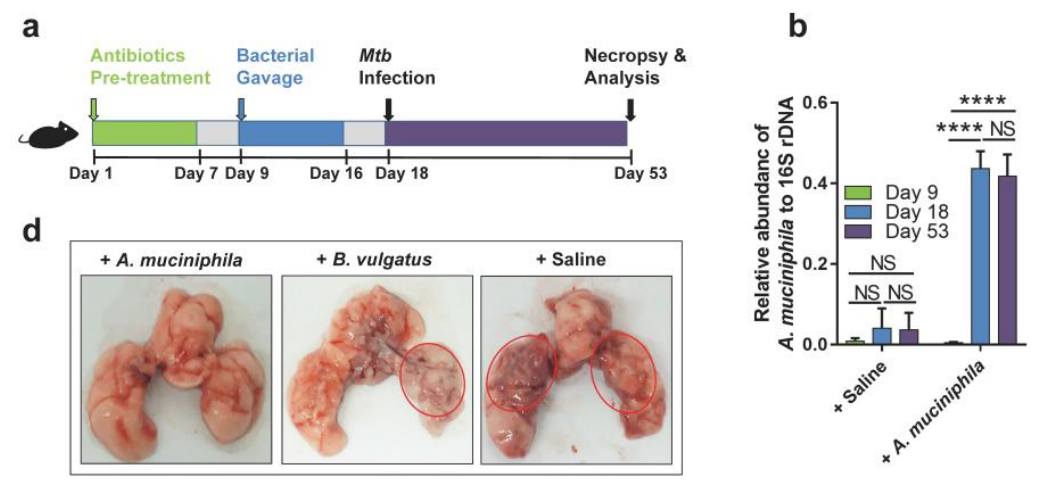

C

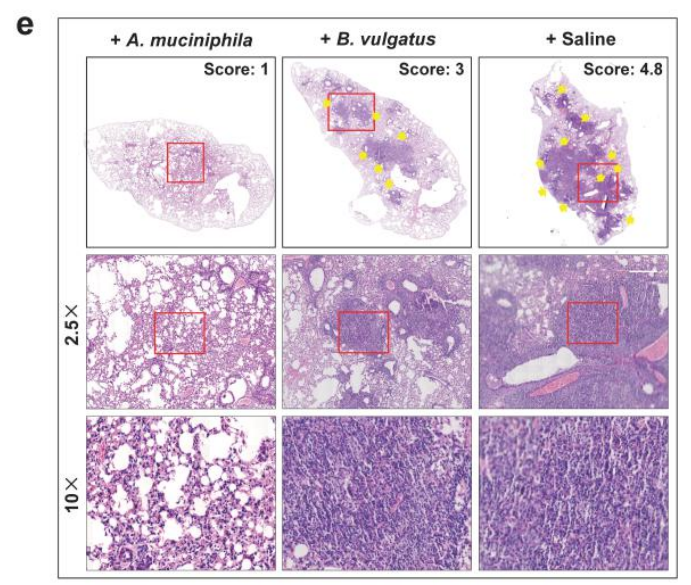

f
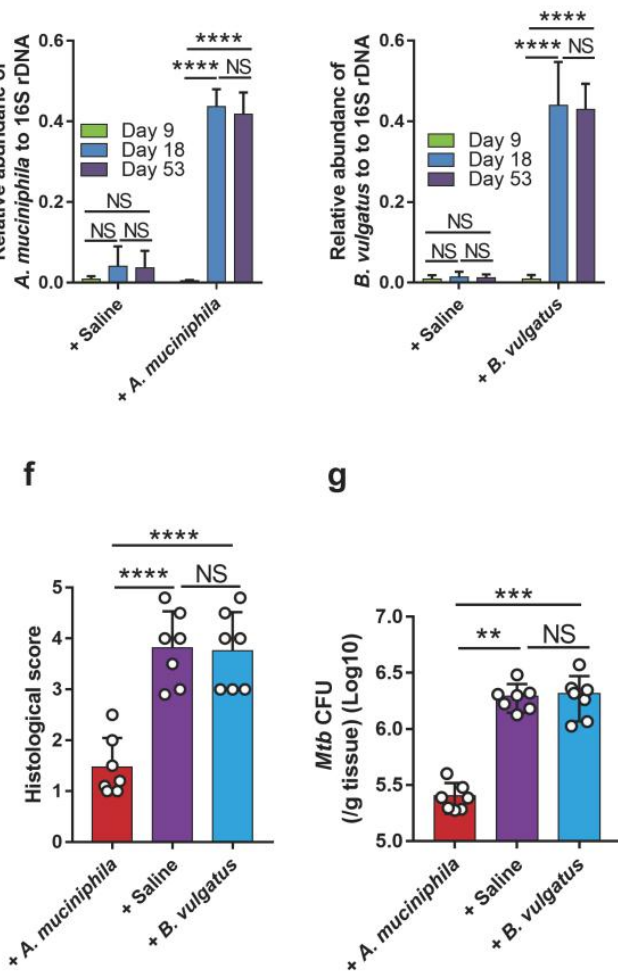

g

h
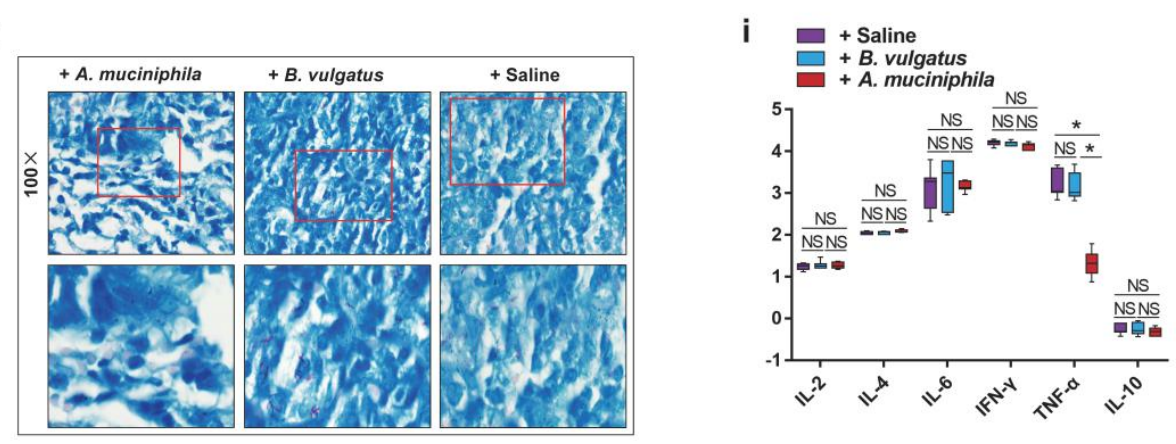

858

Fig. 2. A. muciniphila confers anti-TB protection and reduces TNF- $\alpha$ expression.

860 (a) Experimental diagram for determining the role of A. muciniphila or B. vulgatus during M. tuberculosis infection.

(b-c) Analysis of A. muciniphila (b) or B. vulgatus (c) abundance in stool samples from mice at day 9, 18 and 53 using qPCR analysis.

(d) Three representative lungs of $M$. tuberculosis-infected mice treated with $A$. muciniphila, B. vulgatus or saline. Red circles mark the severe, unresolved 
hemorrhage, massive disruption or caseous necrosis on the lungs of $M$. tuberculosis-infected mice.

(e-f) Hematoxylin and eosin (H\&E) staining of three representative lungs (e) and histological score (f) of M. tuberculosis-infected mice treated with A. muciniphila, B. vulgatus and saline at 5 weeks post infection. Top: original magnification; Middle: $2.5 \times$ of original magnification; Bottom: $10 \times$ of original magnification. 
Fig. 3

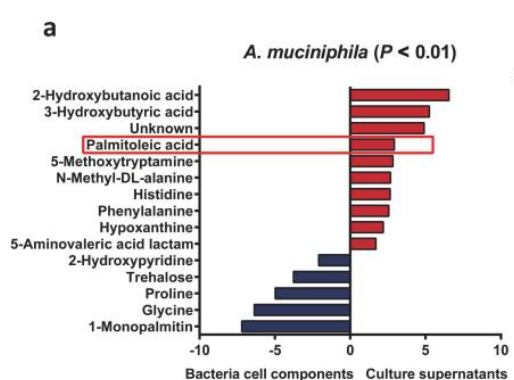

b

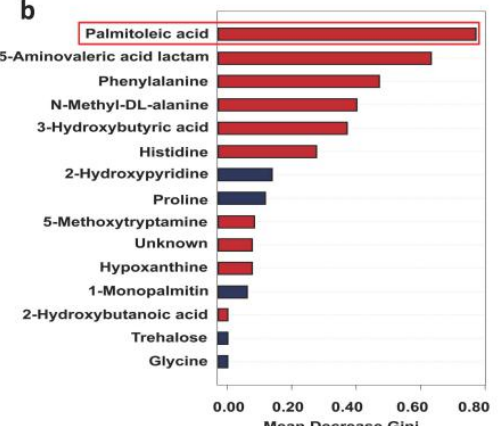

d

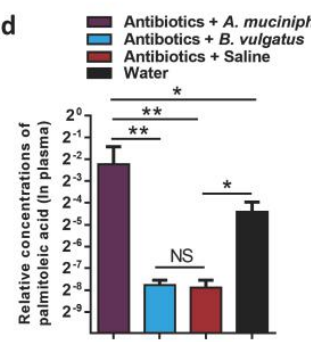

f

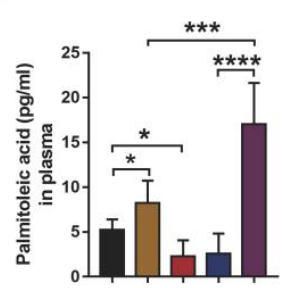

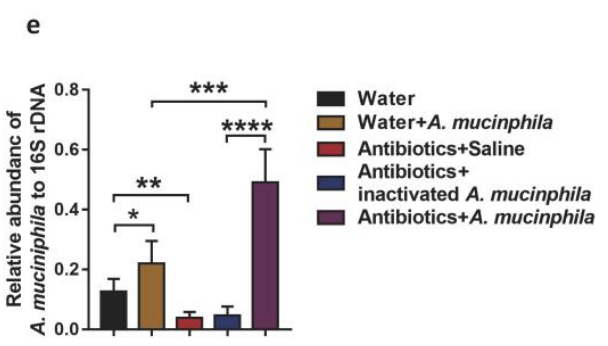

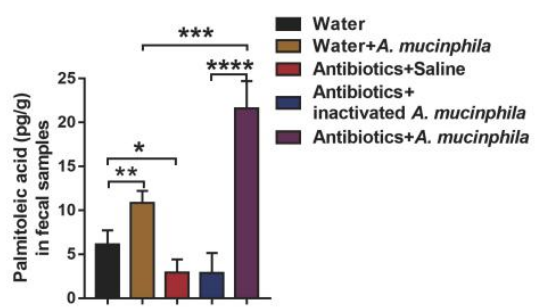

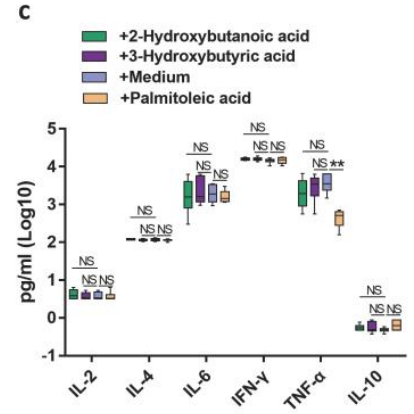

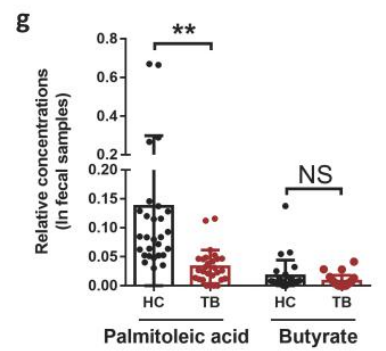

h

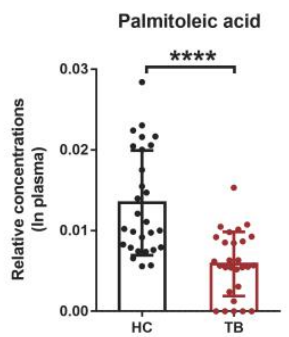

Fig. 3. A. muciniphila produces high levels of palmitoleic acid, an effector metabolite with TNF- $\alpha$ inhibition effects, and healthy controls (HC) show higher palmitoleic acid levels in both feces and plasma than active TB patients (TB).

(a) Palmitoleic acid marked in red box was the ingredient with higher concentrations in culture supernatants of $A$. muciniphila, compared to A. muciniphila bacterial cell ingredients. A. muciniphila was cultured for 72 hours, and then bacteria lysates and culture supernatants were, respectively, subjected to GC-TOF-MSbased metabolic analysis. More ingredients enriched in A. muciniphila culture supernatants with an adjusted $P<0.01$ and $\log 2$ (culture supernatants / bacterial cell ingredients) $>0$; More ingredients enriched in A. muciniphila bacterial cell ingredients with an adjusted $P<0.01$ and $\log 2$ (cultured supernatants / bacterial 
cell ingredients) $<0$. A. muciniphila culture supernatants were normalized to Brain Heart Infusion (BHI). Data are representative of three experiments with three independent biological replicates.

(b) The selected metabolites showing the significant concentration differences between bacterial cell ingredients and culture supernatants of $A$. muciniphila, as determined in (A), showed the most significant influences on Gini of random

(c) Pooled bar graphic data showed the in vitro expression of TNF- $\alpha /$ IFN- $\gamma /$ IL-2/IL4/IL-6/IL-10 in culture supernatants of CD3 $+\mathrm{T}$ cells. $\mathrm{T}$ cells were co-cultured with $M$. tuberculosis lysates $(10 \mu \mathrm{g} / \mathrm{ml})$ only or with 2-Hydroxybutanoic acid, 3Hydroxybutyric acid, or palmitoleic acid for 3 days. $\mathrm{N}=6$ mice per group.

(d) Palmitoleic acid concentrations in plasma derived from mice with oral administration of $A$. muciniphila were higher than those in plasma of mice treated with B. vulgatus or saline or mice with drinking water only. Mice for bacteria transplantation and saline were pretreated with antibiotics for one week and plasma samples were collected at the third day after oral gavage of bacteria. 

were subjected to GC-TOF-MS-based metabolic analysis. $\mathrm{N}=6$ mice per group.

(e) The qPCR-based quantitative analyses of A. muciniphila abundance in stool 
944 way ANOVA with Tukey's multiple comparison test [(c), (d), (e) and (f)] and 945 Student's two-tailed unpaired $t$-test $[(\mathrm{g})$ and $(\mathrm{h})]$. At least two biological repeats were 946 performed.

947 
Fig. 4
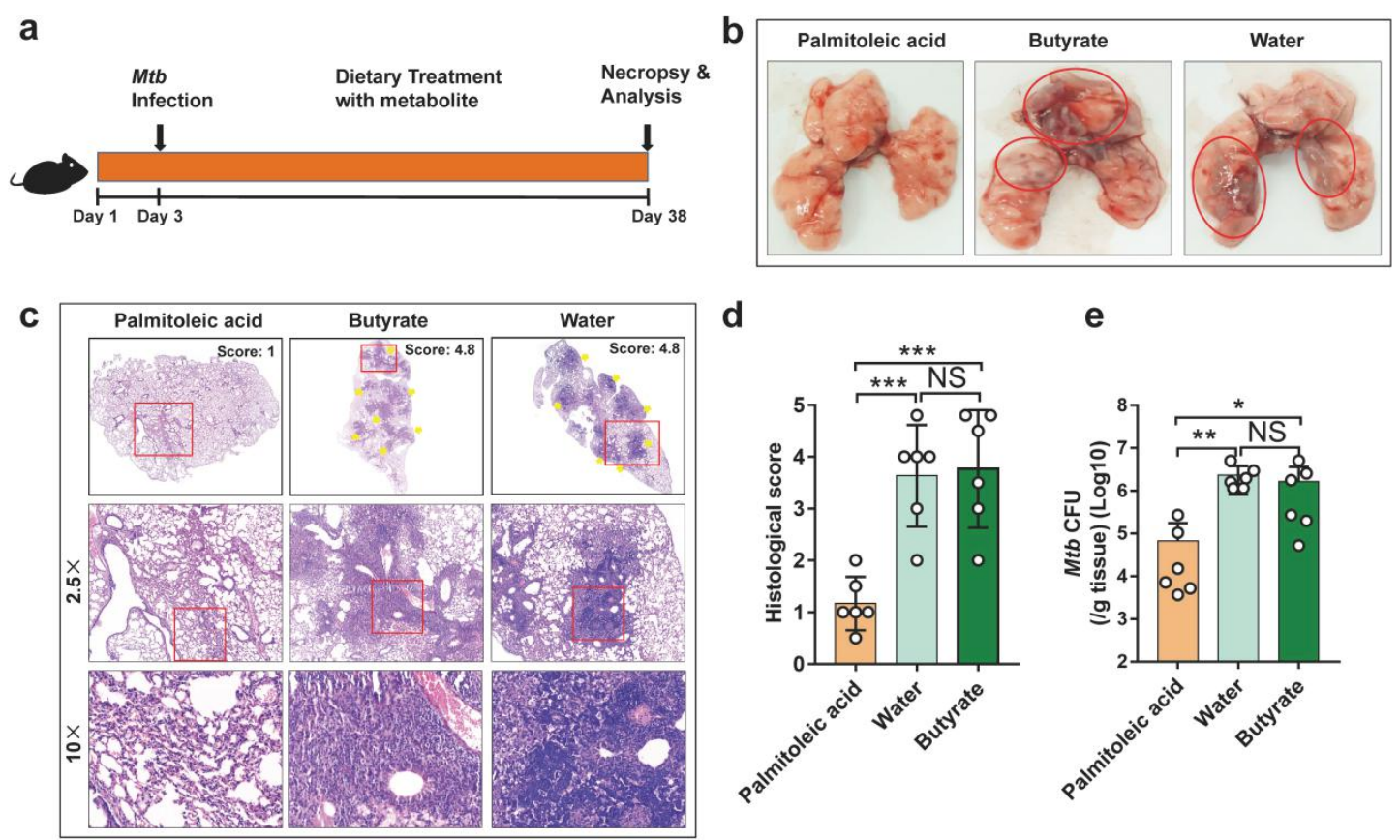

d

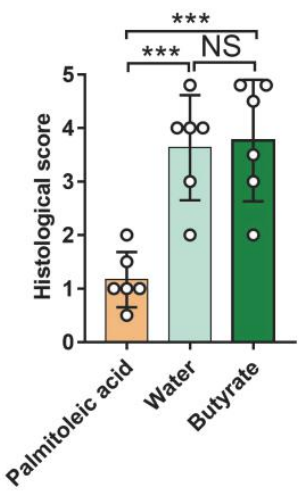

e
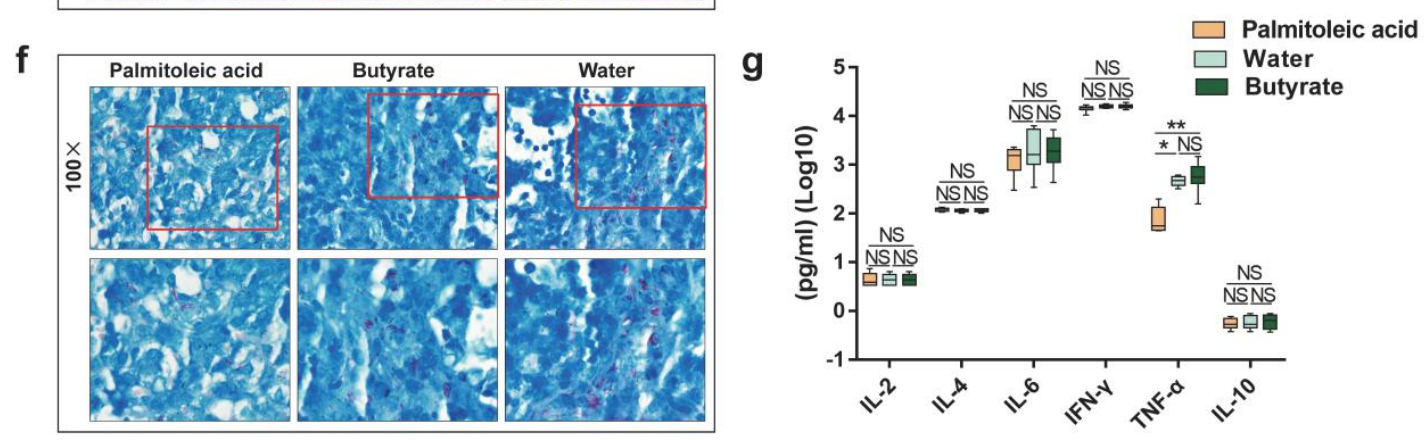

Fig. 4. Dietary an A. muciniphila-derived metabolite, palmitoleic acid, reduces

TB pathology, bacillus burdens and TNF- $\alpha$ production in mice.

(a) Experimental diagram for determining the role of palmitoleic acid or butyrate during M. tuberculosis infection.

(b) Three representative lungs of $M$. tuberculosis-infected mice treated with palmitoleic acid, butyrate or water. Red circles mark the TB pathology or damage

(c-d) H\&E staining of three representative lungs (c) and histological scores (d) of $M$. tuberculosis-infected mice with dietary palmitoleic acid, butyrate or water at 5 
weeks post infection. Top: original magnification; Middle: 2.5 ; Bottom: $10 \times$. The red-boxed areas at the top are enlarged below. Yellow arrowheads mark the lesions and infiltration of inflammatory cells.

(e) Quantitative analysis of bacillus CFU in lung homogenates of M. tuberculosisinfected mice with dietary palmitoleic acid, butyrate or water at 5 weeks post infection.

(f) The M. tuberculosis was visualized in lung sections using acid-fast staining at 5 weeks post infection. Top: $100 \times$ of original magnification. The red-boxed areas at the top are enlarged below.

(g) Pooled bar graphic data show the expression of cytokines in serum derived from M. tuberculosis-infected mice with dietary palmitoleic acid, butyrate or water. $\mathrm{N}=6$ mice per group. Error bars indicate SD. $P<0.05(*) ; P<0.01(* *) ; P<0.001$

971 with Tukey's multiple comparison test [(d), (e) and (g)]. At least two biological 972 repeats were performed.

973 
Fig. 5
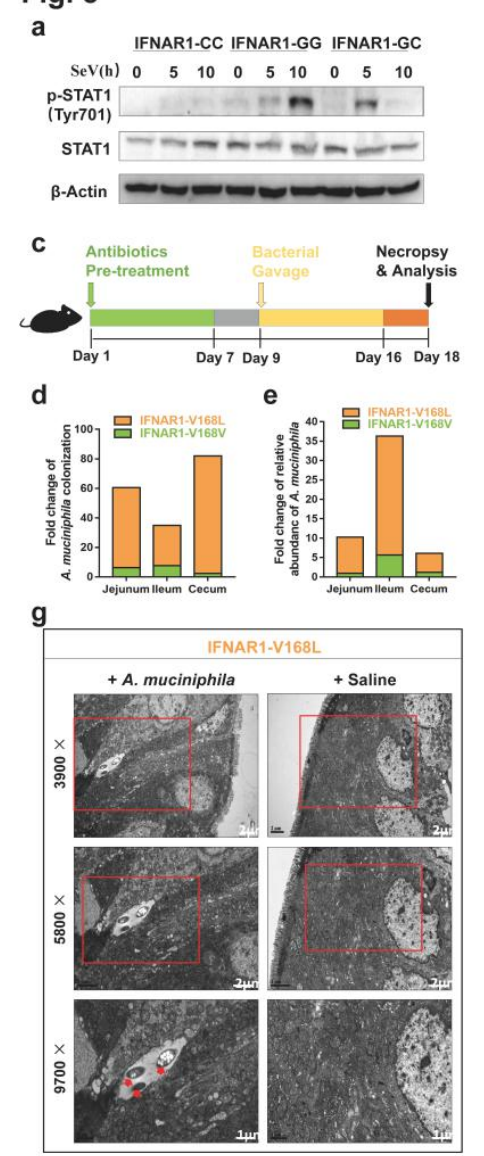

f 吕 IFNARA-V1686 $h$
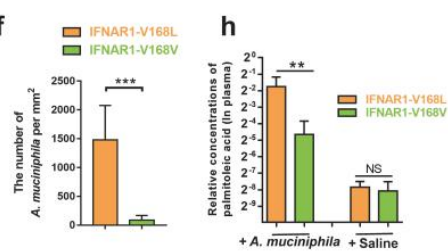

b
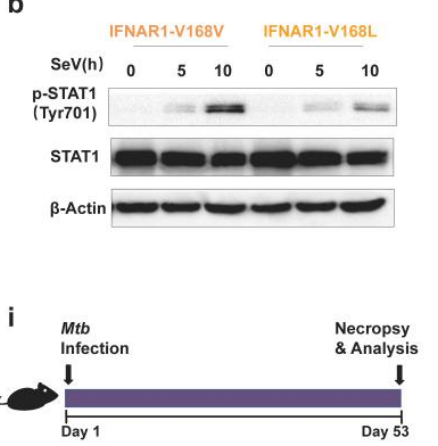

j

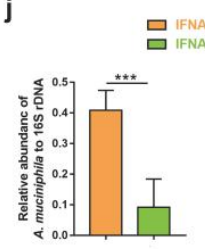

m
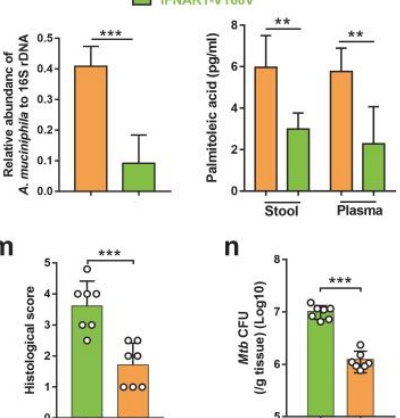

n
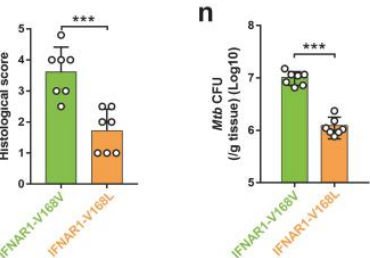

0

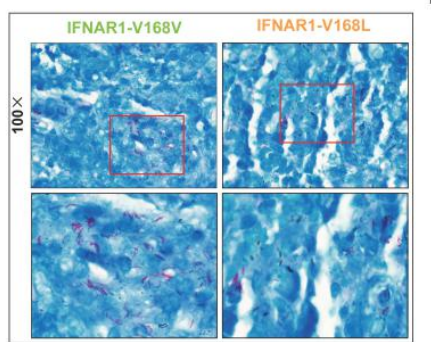

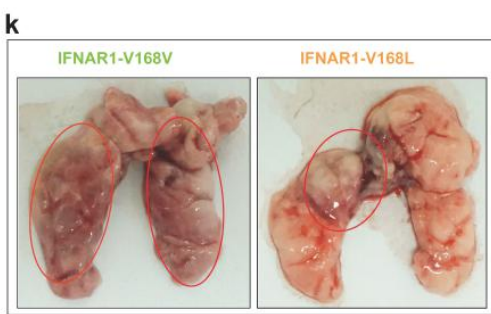
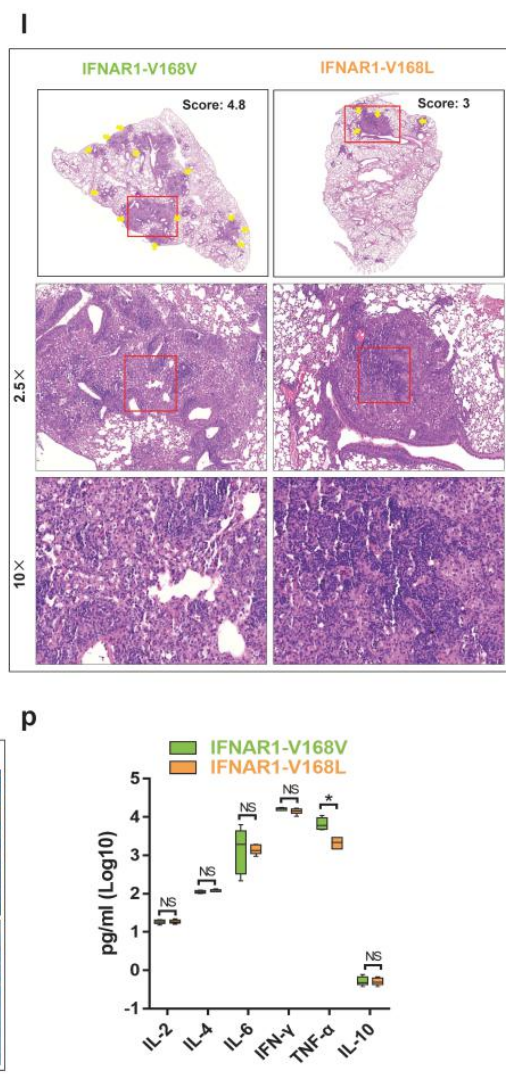

Fig. 5. Ifnar1 rs2257167 $G$ allele dictates stronger IFN-I signaling in both humans and mice, decreases colonization and abundance of $A$. muciniphila, and promotes higher TNF- $\alpha$ production and severer TB infection in transgenic mouse models.

(a) Immunoblot analysis of the levels of phosphorylated(p)- and total STAT1 in PBMCs derived from individuals carrying genotype GG, GC, CC with Sendai virus stimulation at 0,5 and 10 hours. Data are representative of three experiments with three independent biological replicates. 
983

984

985

986

987

988

989

990

991

992

993

(b) Immunoblot analysis of the levels of p- and total STAT1 in Bone Marrow Derived Macrophage (BMDM) derived from IFNAR1-V168V and IFNAR1-V168L mice at 0,5 and 10 hours following Sendai virus stimulation. Data are representative of three experiments with three independent biological replicates.

(c) Experiment design: oral administration for determining the role of IFN-I signaling in A. muciniphila abundance.

(d) Pooled data of relative amounts of A. muciniphila recovered from jejunum, ileum and cecum of IFNAR1-V168L (orange bar) and IFNAR1-V168V (green bar) mice, respectively. Fold changes were calculated as: $\mathrm{CFU}$ of $A$. muciniphila mice monocolonized with A. muciniphila normalized to those of saline-treated mice carrying the same genotype. $\mathrm{N}=20$ mice per group.

(e) Pooled data of relative expression levels of A. muciniphila recovered from jejunum, ileum and cecum of IFNAR1-V168L (orange bar) and IFNAR1-V168V (green bar) mice, respectively. Fold changes were calculated as: expression levels of A. muciniphila of mice mono-colonized with A. muciniphila normalized to those of same genotype mice with oral saline administration. $\mathrm{N}=20$ mice per group.

(f) FISH/Confocal microscope-based half-quantitative assessment of A. muciniphila on the intestinal mucosa of IFNAR1-V168L and IFNAR1-V168V mice monocolonized with A. muciniphila. Twenty confocal microscopic views from each mouse line were subjected to analysis of A. muciniphila amounts.

(g) Representative TEM images of intestinal samples from IFNAR1-V168L mice 
gavaged with $A$. muciniphila and saline, respectively. The red-boxed areas at the upper panel are enlarged lower panel. $2 \mu \mathrm{m}$ (Top panel), $2 \mu \mathrm{m}$ (Middle panel) and $1 \mu \mathrm{m}$ (Bottom panel). Red arrowheads mark the A. muciniphila.

(h) Palmitoleic acid concentrations in plasma derived from IFNAR1-V168L mice were higher than in plasma of IFNAR1-V168V mice when these mice were orally subjected to GC-TOF-MS-based metabolic analysis. $\mathrm{N}=6$ mice per group.

(i) Experimental diagram for determining the role of SNP rs2257167 during $M$. tuberculosis infection.

(j) Analysis of A. muciniphila abundance in stool samples using qPCR analysis, and the quantitative analyses of palmitoleic acid concentrations in plasma and fecal samples derived from IFNAR1-V168V and IFNAR1-V168L mice at 5 weeks post infection.

(k) Representative lungs derived from the M. tuberculosis-infected IFNAR1-V168V and IFNAR1-V168L mice, respectively. Red circles mark the hemorrhage or necrosis in the lungs of $M$. tuberculosis-infected mice.

(l-m) H\&E staining of two representative lungs (L) and histological score (M) of $M$. tuberculosis-infected transgenic mice at 5 weeks post infection. Top: original magnification; Middle: $2.5 \times$ of original magnification; Bottom: $10 \times$ of original magnification. The red-boxed areas at the top are enlarged below. Yellow 
arrowheads point lesions and infiltration of inflammatory cells.

1028

1029

1030

1031

1032

1033

1034

1035

1036

1037

1038

1039

(n) CFU quantification of $M$. tuberculosis in the lungs at 5 weeks post infection.

(o) The acid-fast staining-based assessment of M. tuberculosis inside the lung tissues. Note that more acid-fast staining-positive bacilli in lung sections derived from IFNAR1-V168V mice. Top: $100 \times$ of original magnification. The red-boxed areas at the top are enlarged below.

(p) Pooled bar graphic data show the expression of cytokines in serum derived from M. tuberculosis-infected IFNAR1-V168V and IFNAR1-V168L mice, respectively. Serum was collected on 35 days post M. tuberculosis infection for CBA-based cytokine analysis.

Error bars indicate SD. $P<0.05(*) ; P<0.01(* *) ; P<0.001(* * *) . P$ value was calculated by Student's two-tailed unpaired $t$-test [(f), (h), (j), (m), (n) and (p)]. At least two biological repeats were performed. 
Fig. 6
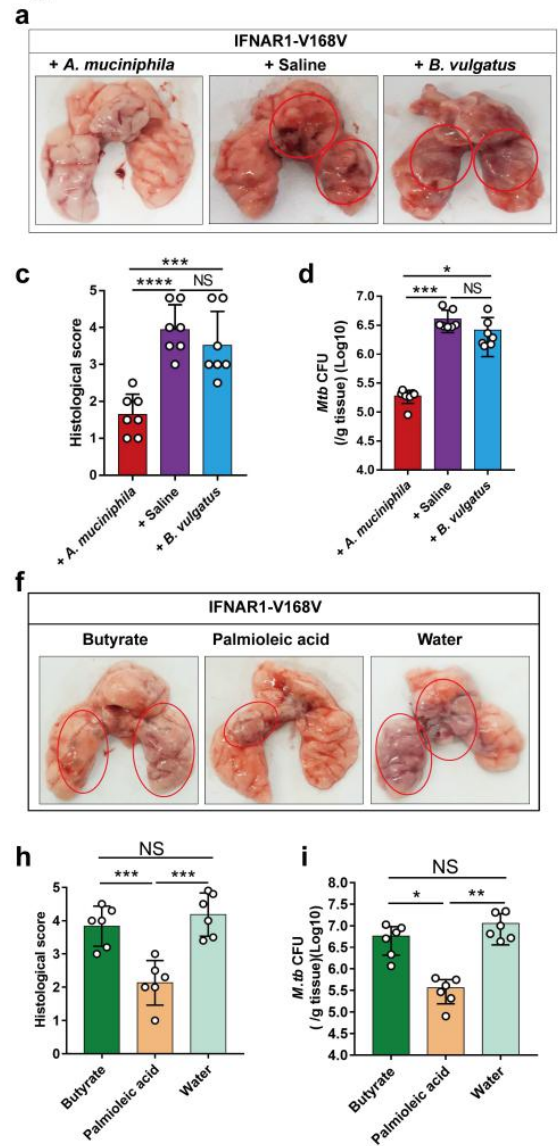

b
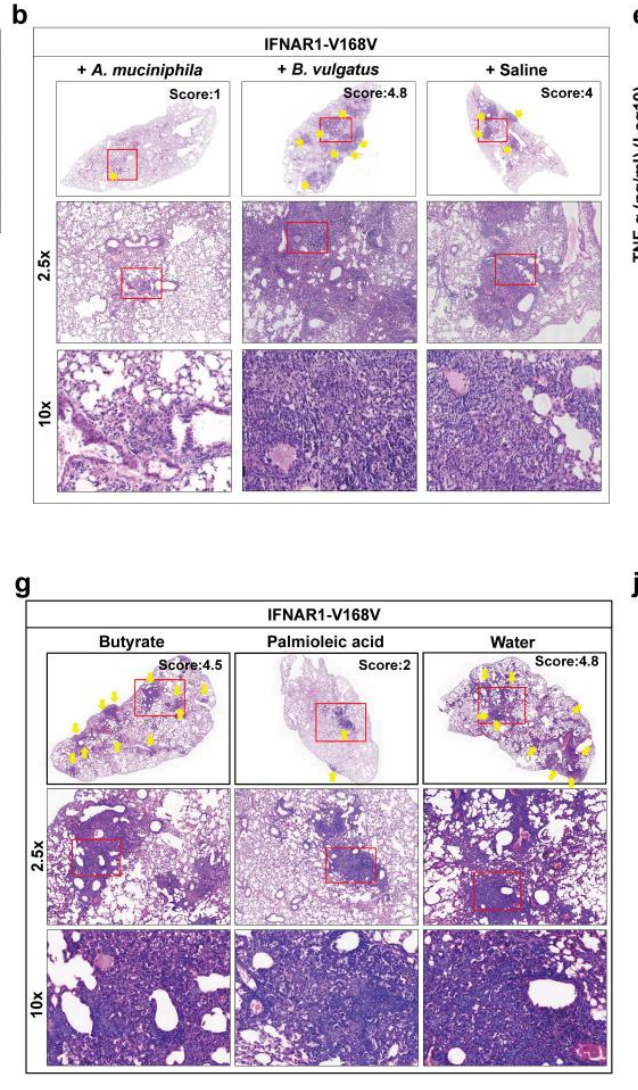

e
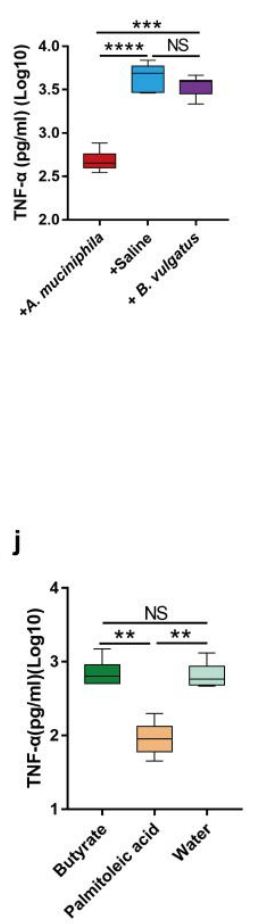

1040

Fig. 6. Oral gavage of $A$. muciniphila or palmitoleic acid reduces TB pathology and bacillus burdens in M. tuberculosis-infected transgenic mice carrying human Ifnar1 rs2257167 "G" allele (termed IFNAR1-V168V mice).

(a) Three representative lungs derived from M. tuberculosis-infected IFNAR1-V168V mice with oral administration of $A$. muciniphila, B. vulgatus and saline. Red circles mark the lung pathology of infected mice.

(b-c) H\&E staining of three representative lungs (b) and histological score (c) of $M$. tuberculosis-infected IFNAR1-V168V mice at 5 weeks post infection. Top: original magnification; Middle: $2.5 \times$; Bottom: $10 \times$. The red-boxed areas at the top are enlarged below. Yellow arrowheads mark lesions and infiltration of inflammatory cells, compared with saline treatment. $\mathrm{N}=7$ mice per group. 
1052

1053

1054

1055

1056

1057

1058

1059

1060

1061

1062

1063

1064

1065

1066

1067

1068

1069

1070

1071

1072

1073

(d) CFU quantification analysis of M. tuberculosis burdens in the lung homogenates at 5 weeks post infection. IFNAR1-V168V mice with mono-colonization of $A$. muciniphila but not $B$. vulgatus had much lower bacterial burdens, compared with saline treatment. $\mathrm{N}=7$ mice per group.

(e) Pooled bar graphic data show the expression of TNF- $\alpha$ in serum derived from $M$. tuberculosis-infected IFNAR1-V168V mice with oral administration of $A$. muciniphila, B. vulgatus and saline. Serum was collected on 35 days post $M$. tuberculosis infection for CBA-based cytokine analysis. $\mathrm{N}=7$ mice per group.

(f) Gross pathology shows that the palmitoleic acid decreases hemorrhage and necrosis in lungs of $M$. tuberculosis-infected IFNAR1-V168V mice at 5 weeks post infection. Red circles mark the TB pathology or damage on the lungs of $M$. tuberculosis-infected mice.

(g-h) H\&E staining of three representative lungs (g) and histological scores (h) of $M$. tuberculosis-infected IFNAR1-V168V mice with dietary palmitoleic acid, butyrate or water at 5 weeks post infection. Top: original magnification; Middle: 2.5; Bottom: $10 \times$. The red-boxed areas at the top are enlarged below. As marked by yellow arrowheads, mice with dietary palmitoleic acid but not butyrate showed milder lesions and less infiltration of inflammatory cells, compared with water treatment. $\mathrm{N}=6$ mice per group.

(i) Quantitative analysis of bacillus CFU in lung homogenates derived from $M$. tuberculosis-infected IFNAR1-V168V mice with dietary palmitoleic acid, butyrate or water at 5 weeks post infection. $\mathrm{N}=6$ mice per group. 
1074 (j) Pooled bar graphic data show the expression of TNF- $\alpha$ in serum derived from $M$. 1075 tuberculosis-infected IFNAR1-V168V mice with dietary palmitoleic acid, 1076 butyrate or water. Serum was collected on 35 days post M. tuberculosis infection 1077 for CBA-based cytokine analysis. $\mathrm{N}=6$ mice per group.

1078 Error bars indicate SD. $P<0.05\left(^{*}\right) ; P<0.01(* *) ; P<0.001(* * *) ; P<0.0001$ $1079(* * * *)$; NS (no statistical significance). $P$ values were calculated by one-way 1080 ANOVA with Tukey's multiple comparison test [(c) to (e), (h) to (j)]. At least two 1081 biological repeats were performed.

1082 
Fig. 7
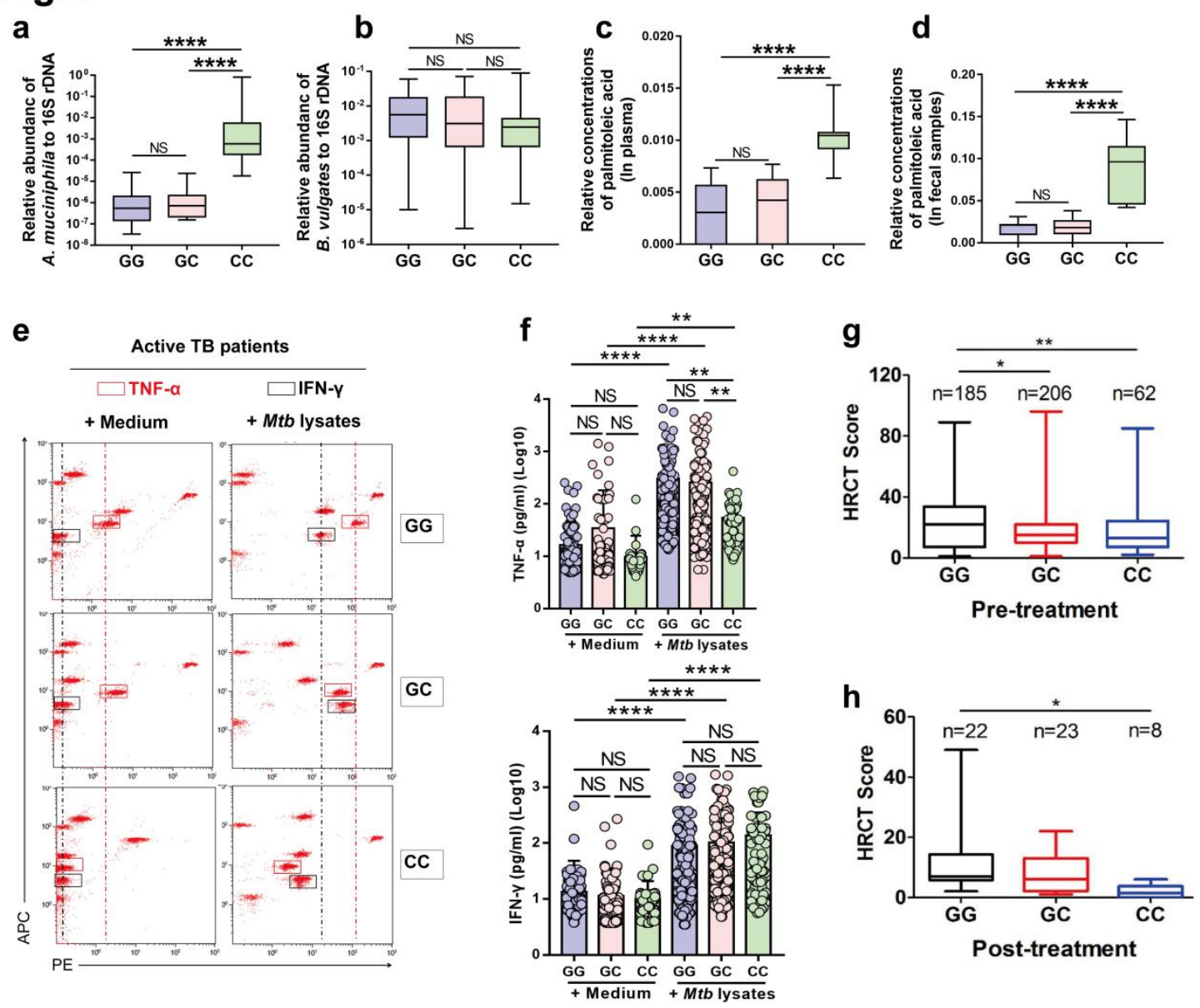

Fig. 7. Ifnar1 rs2257167 $\mathrm{G}$ allele is associated with less $A$. muciniphila abundance,

decreased levels of palmitoleic acid, higher TNF- $\alpha$ production, severer TB

pathology and poorer prognosis in humans with active TB disease.

(a-b) Bar graphic data shows the qPCR-based relative abundance of $A$. muciniphila (a) and B. vulgatus (b) in fecal samples derived from TB patients carrying genotype GG, GC and CC. Patients were from Shenzhen cohort.

(c-d) Bar graphic data shows the Relative concentrations of palmitoleic acid in plasma (c) and fecal samples (d) derived from TB patients carrying genotype GG, GC and (e) Representative CBA analysis of culture supernatants of PBMCs derived from TB 
patients carrying genotype GG, GC, CC. Patients were from Shenzhen cohort. PBMCs were cultured in presence or absence of M. tuberculosis lysates $(10 \mu \mathrm{g} / \mathrm{ml})$ for 3 days, and supernatants were then subjected to CBA analysis to determine the expression of TNF- $\alpha / \mathrm{IFN}-\gamma / \mathrm{IL}-2 / \mathrm{IL}-4 / \mathrm{IL}-6 / \mathrm{IL}-10$. Red and black-boxed areas mark the fluorescent clusters of TNF- $\alpha$ and IFN- $\gamma$, respectively, and dashed lines mark the shift of fluorescent clusters of TNF- $\alpha$ and IFN- $\gamma$, respectively.

(f)Pooled bar graphic data shows the expression of TNF- $\alpha$ (upper panel) and IFN- $\gamma$ (lower panel), respectively, in culture supernatants of PBMCs derived from TB chemotherapy.

(h) Paired analysis of HRCT score in 2 years after anti-TB chemotherapy in pulmonary TB patients carrying Ifnarl rs2257167 genotype GG $(\mathrm{n}=22), \mathrm{GC}(\mathrm{n}=$ $23)$, and $\mathrm{CC}(\mathrm{n}=8)$.

Each paired data set represents an individual patient. GG $(\mathrm{n}=185), \mathrm{GC}(\mathrm{n}=206)$ and $\mathrm{CC}(\mathrm{n}=62)$ for $(\mathrm{A})$ to $(\mathrm{G})$. Error bars indicate SD. $P<0.05(*) ; P<0.01(* *) ; P<$ $0.0001(* * * *)$; NS (no statistical significance). One-way ANOVA with Newman-

1114 Keuls multiple comparison test [(a) to (d), (f) to (h)]. At least two biological repeats were performed. 


\section{Extended Data Fig. 1}
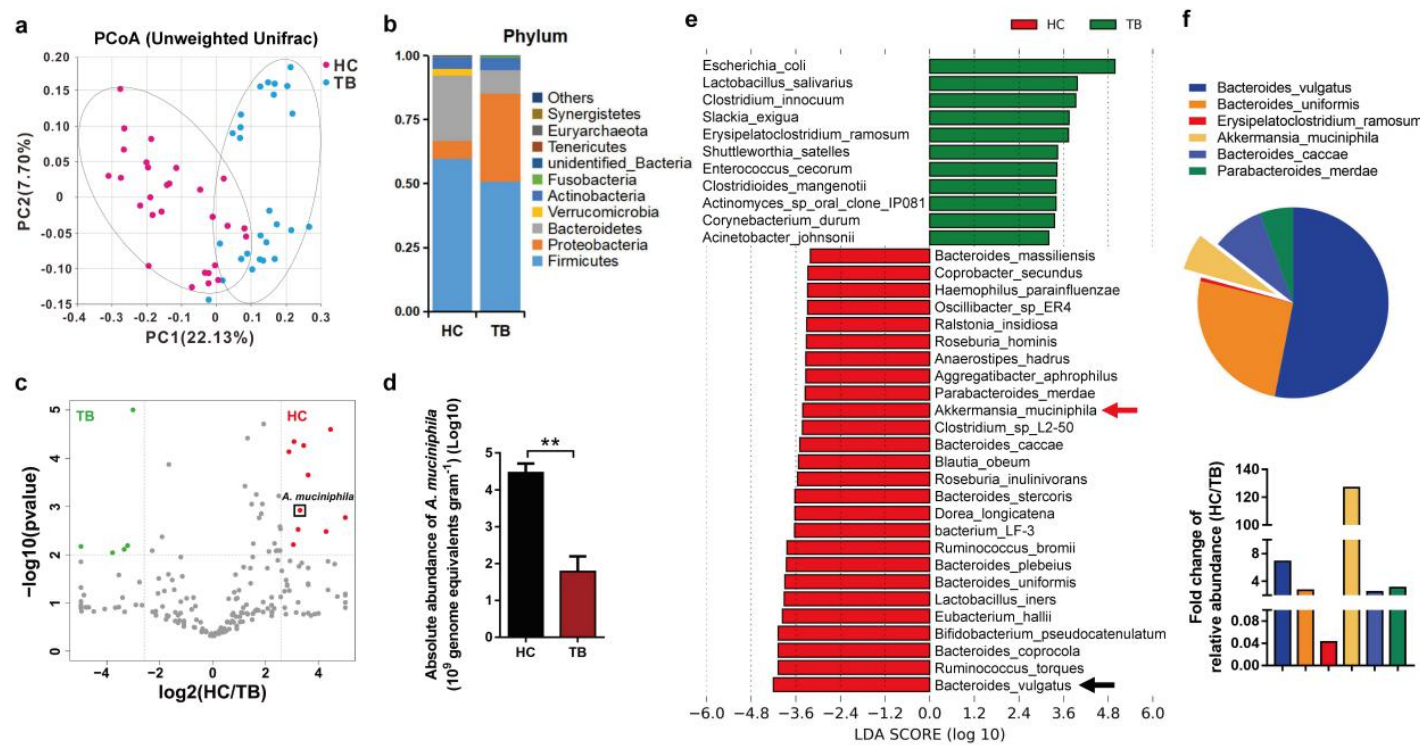

d

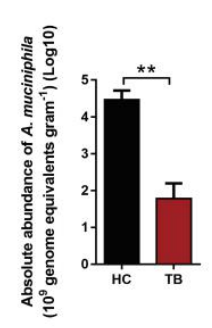

Extended Data Fig. 1. Healthy controls (HC) and active TB patients (TB) exhibit

\section{significant differences in diversity, composition, and abundance of gut bacteria}

\section{at Shenzhen cohort.}

(a) PCoA plot (based on Unweighted UniFrac distances). Red points indicated the

(b) The relative abundance of gut bacteria at phylum level in the fecal samples from $\mathrm{HC}(\mathrm{n}=28)$ and TB $(\mathrm{n}=26)$.

(c) Volcano plot of all species found in fecal samples. Red points indicated species with an adjusted $P<0.01$ and $\log 2(\mathrm{HC} / \mathrm{TB})>2.5$; green points indicated species with an adjusted $P<0.01$ and $\log 2(\mathrm{HC} / \mathrm{TB})<-2.5$. A. muciniphila, as marked with black box, was a more abundant species enriched in HC.

(d) The absolute abundance of detected $A$. muciniphila in the fecal samples from HC $(\mathrm{n}=28)$ and TB $(\mathrm{n}=26)$. 

abundant species in $\mathrm{HC}$ are shaded with red. Red arrowhead marks the $A$. muciniphila, black arrowhead marks B. vulgatus.

(f) Circle charts marked the relative abundance of six bacteria with differentiated relative abundance between $\mathrm{HC}$ and TB in Shenzhen cohort, and these A. muciniphila, B. caccae, P. merdae, and E. ramosun between $\mathrm{HC}$ and $\mathrm{TB}$ in Shenzhen cohort (fold changes were calculated as $\mathrm{HC} / \mathrm{TB}$ ). Bacteria are identified by color bars above the chart.

1145 Error bars indicate SD. $P<0.01(* *) . P$ values were calculated by Mann-Whitney U 1146 test $[(\mathrm{c})$ and $(\mathrm{d})]$. 
Extended Data Fig. 2

a

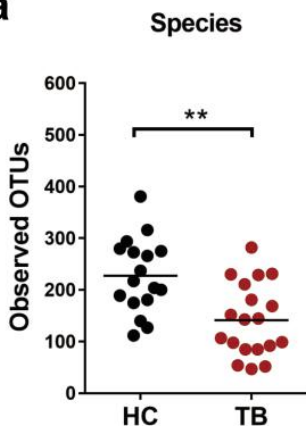

b

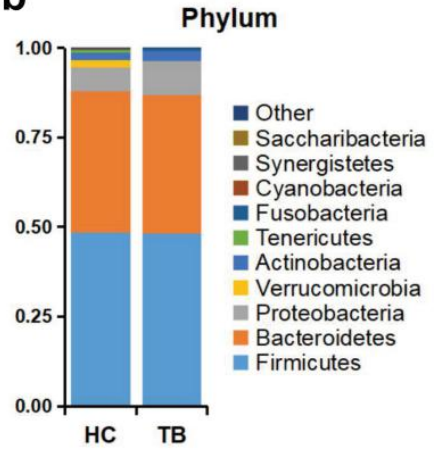

Top 10 species $(p<0.05)$

d

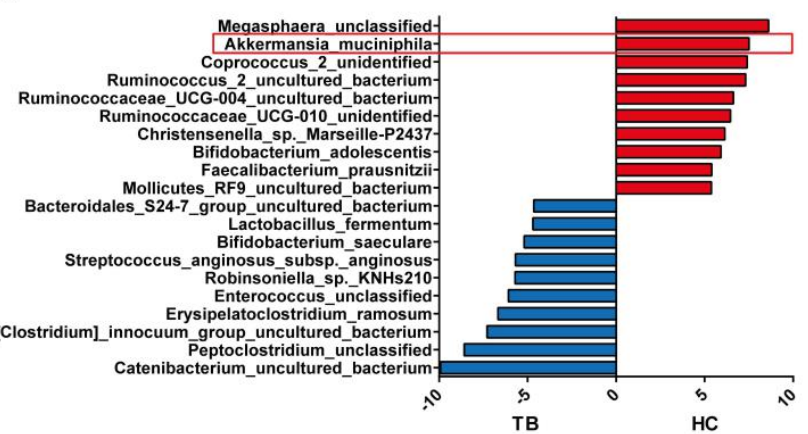

g

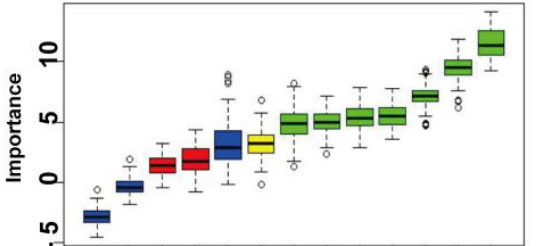

Alloprevotella uncultured organism Erysipelatoclostridium_ramosum Bacteroides_thetaiotaomicro

Parabacteroides_distasonis

Clostridium innocuum_uncultured_bacterium Bacteroides ovatus

Peptoclostridium_unclassified

Parasutterella_unclassified
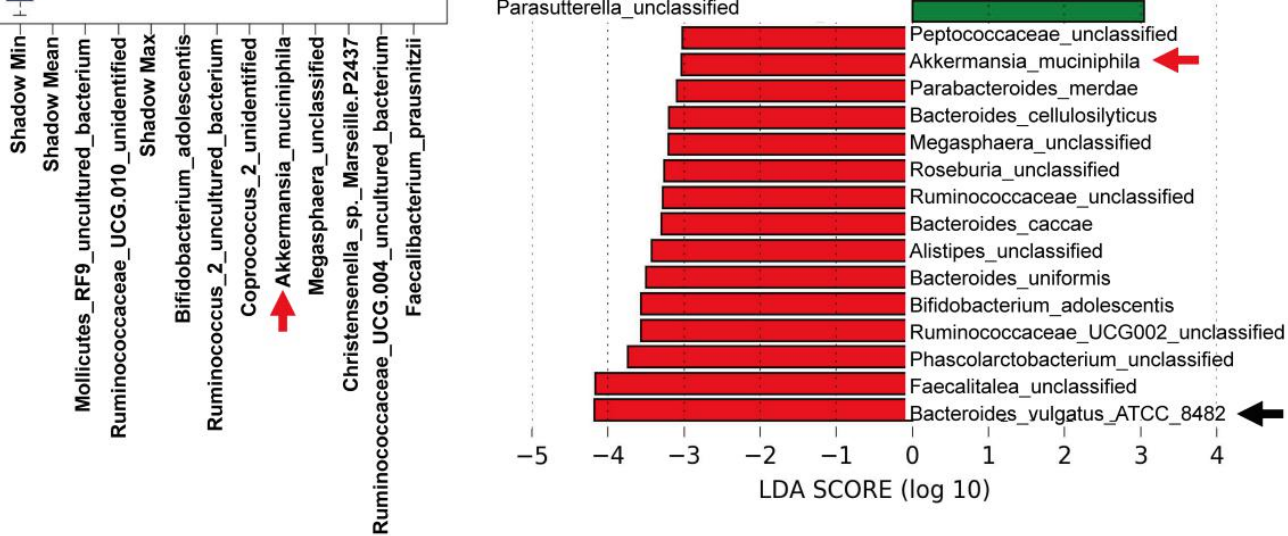

h

i

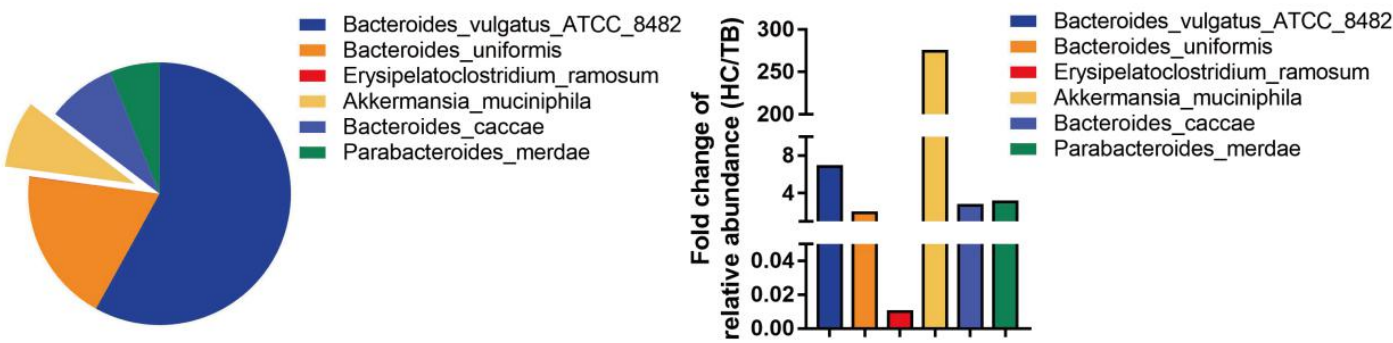

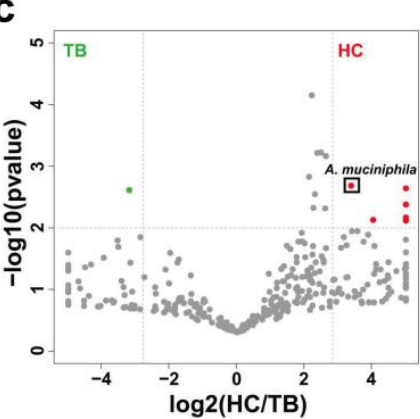

e

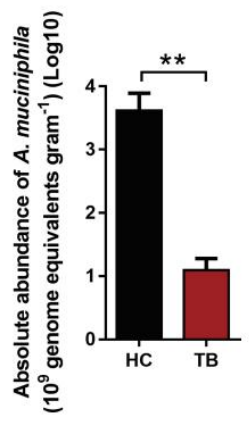



composition and abundance of gut bacteria, and abundance of $A$. muciniphila and $B$. vulgatus are significantly higher in $\mathrm{HC}$ than TB at Foshan cohort.

(a) Number of observed OTUs of species in fecal microbiota from $\mathrm{HC}(\mathrm{n}=17)$ and

(b) The relative abundance of gut bacteria in phylum level in the fecal samples from $\mathrm{HC}(\mathrm{n}=17)$ and TB $(\mathrm{n}=19)$.

(c) Volcano plot of all species found in fecal samples. Red points indicated species with an adjusted $P<0.01$ and $\log 2(\mathrm{HC} / \mathrm{TB})>2.5$; green points indicated species with an adjusted $P<0.01$ and $\log 2(\mathrm{HC} / \mathrm{TB})<-2.5$. A. muciniphila pointed in black box was the species with higher abundance enriched in $\mathrm{HC}$.

(d) A. muciniphila was belonged to top 10 bacterial species in fecal microbiota of HC. Species with enriched relative abundance in $\mathrm{HC}$ are adjusted $P<0.05$ and $\log 2$ $(\mathrm{HC} / \mathrm{TB})>0$, species with enriched relative abundance in TB are adjusted $P<$ 0.05 and $\log 2(\mathrm{HC} / \mathrm{TB})<0$. The red-boxed area marks the $A$. muciniphila.

(e) The absolute abundance of $A$. muciniphila in the fecal microbiota from $\mathrm{HC}(\mathrm{n}=17)$ and $\operatorname{TB}(\mathrm{n}=19)$.

(f) Predictive power of top 10 species enriched in $\mathrm{HC}$ (i.e. the top 10 most reduced species in TB) assessed by random forest analysis. Blue boxplots correspond to minimal, average, and maximum Z-score of shadow species, which were shuffled version of real species introduced to random forest classifier and act as benchmarks to detect truly predictive species. Red boxplots represent rejected 

species, yellow boxplots represent suggestive species, and green boxplots represent confirmed species. The red arrowhead marks the $A$. muciniphila.

(g) Histogram of the Linear discriminant analysis (LDA) coupled with effect size measurements (LEfSe) identified the species with different abundance in $\mathrm{HC}$ and pointed B. vulgatus.

(h) Circle charts showed the relative abundance of six bacteria with differentiated

(i) Histogram showed the fold change of relative abundance of six bacteria, including B. vulgatus, B. uniformis, A. muciniphila, B. caccae, P. merdae and E. ramosun between $\mathrm{HC}$ and TB ( calculated as $\mathrm{HC} / \mathrm{TB}$ ) in Foshan cohort.

1183 Error bars indicate SD. $P<0.01(* *) . P$ value was calculated by Mann-Whitney test 1184 [(a), (c), (d) and (e)]. 


\section{Extended Data Fig. 3}
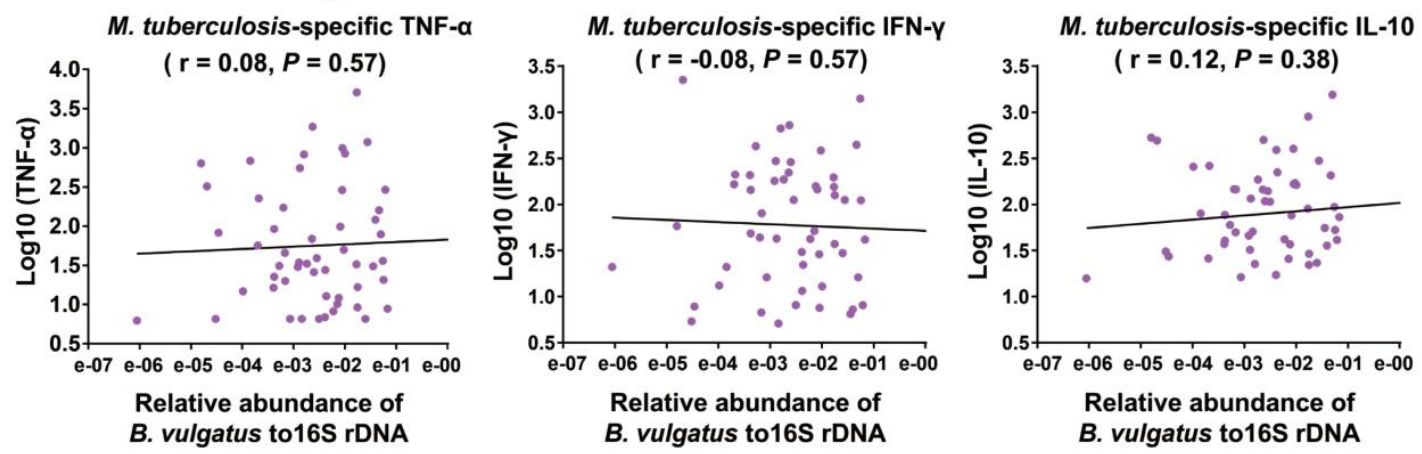

1187 Extended Data Fig. 3. The association of $M$. tuberculosis-specific cytokines ex vivo produced by PBMCs and B. vulgatus in fecal samples of TB patients.

1189 Spearman correlation analysis showed the association between TNF- $\alpha$, IFN- $\gamma$, or

1190 IL-10 and the relative abundance of $B$. vulgatus. There were no significant correlation 1191 between $B$. vulgatus and $M$. tuberculosis-specific TNF- $\alpha, M$. tuberculosis-specific 1192 IFN- $\gamma$ or $M$. tuberculosis-specific IL-10. $P$-value was calculated by Spearman 1193 correlation. 


\section{Extended Data Fig. 4}

a

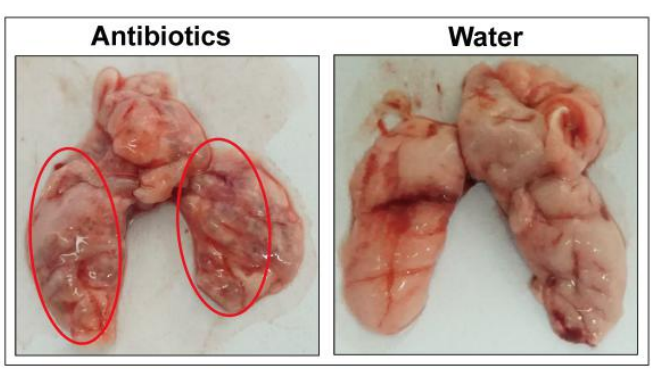

b

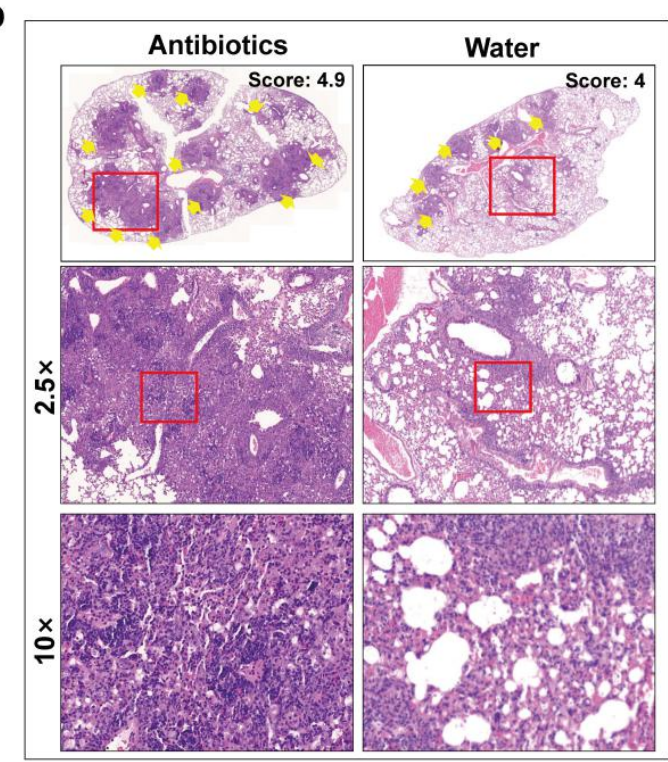

C

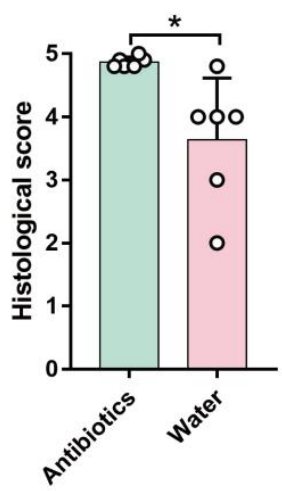

e

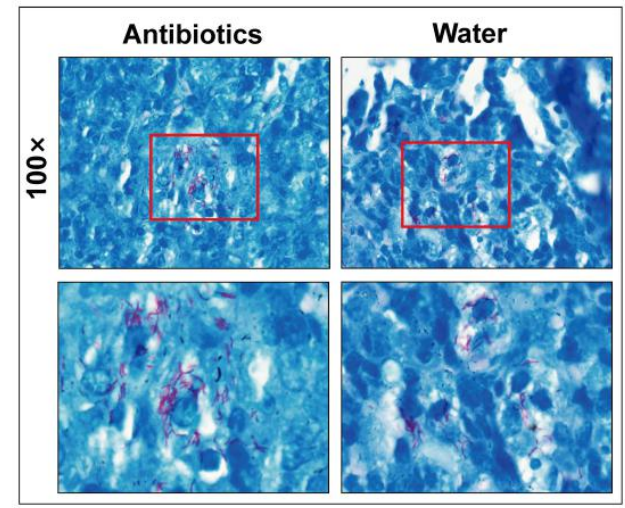

Extended Data Fig. 4. Antibiotics-treated mice show much severer pulmonary pathology and higher bacillus burdens during M. tuberculosis infection.

(a) Two representative lungs derived from $M$. tuberculosis-infected mice with antibiotics or water control treatment. Red circles mark the cystic changes, hemorrhage or necrosis on the lungs of infected mice.

(b-c) Hematoxylin and eosin (H\&E) staining of two representative lungs (b) and the histological scores (c) of M. tuberculosis-infected mice with antibiotics or water control treatment at 5 weeks post infection. Top: original magnification; Middle: $2.5 \times$ of original magnification; Bottom: $10 \times$ of original magnification. The redboxed areas at the top are enlarged below. As marked by yellow arrowheads, 
antibiotics-treated mice showed much severer lesions and more infiltration of inflammatory cells than untreated mice.

(d) Quantification analysis of $M$. tuberculosis CFU in the lung homogenates of $M$. tuberculosis-infected mice at 5 weeks post infection.

(e) The acid-fast staining of $M$. tuberculosis in lung section of mice at 5 weeks post infection. Note that more acid-fast staining-positive bacilli in lung sections derived from antibiotics-treated mice. Top: $100 \times$ of original magnification. The red-boxed areas at the top are enlarged below.

$1213 \mathrm{~N}=6$ mice per group. Error bars indicate SD. $P<0.05\left(^{*}\right)$ and $P<0.01\left({ }^{* *}\right) . P$ values 1214 were calculated by Student's two-tailed unpaired $t$-test [(c) and (d)]. At least two 1215 biological repeats were performed. 


\section{Extended Data Fig. 5}

a

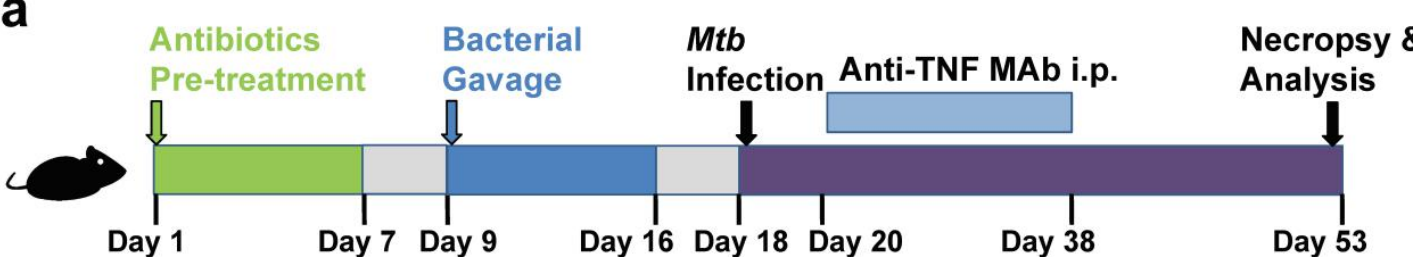

b

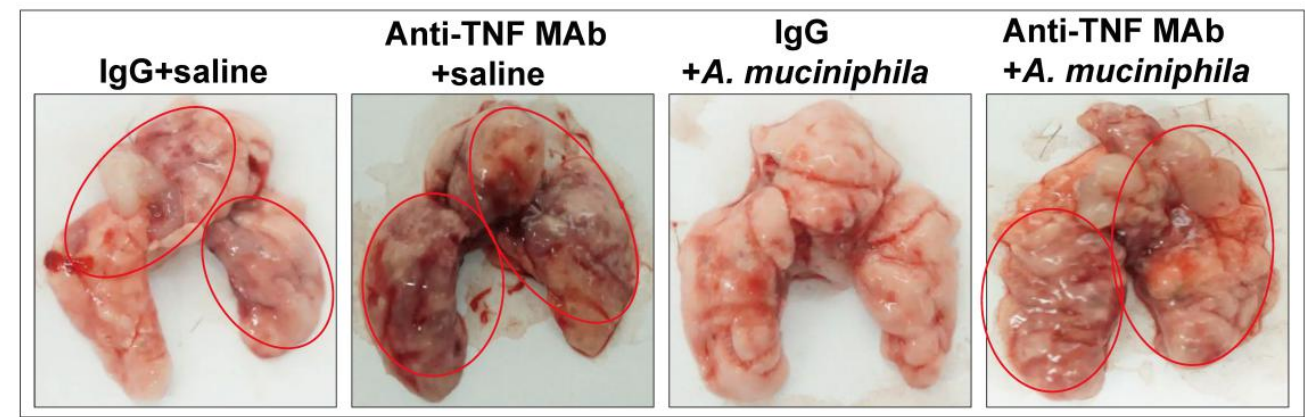

c

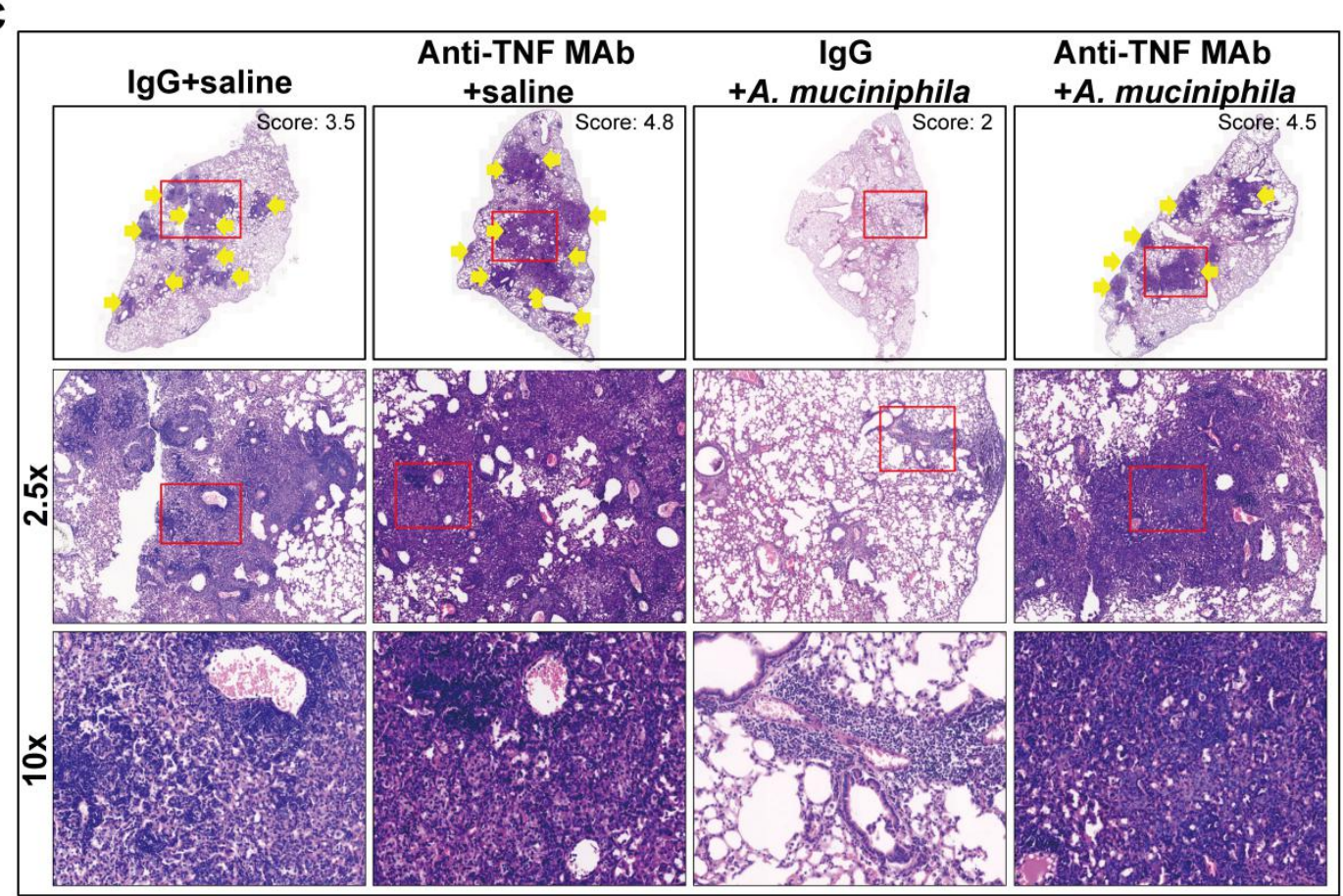

d

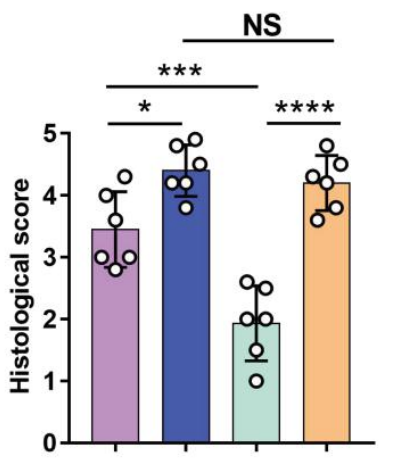

e

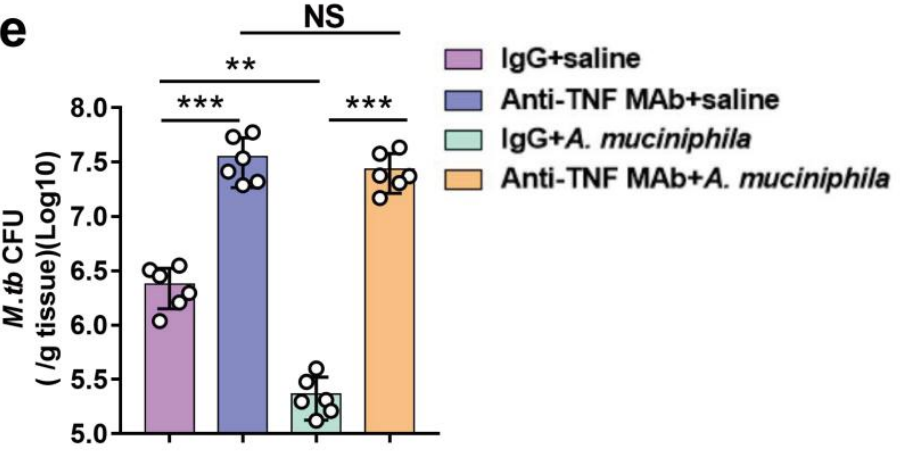



and bacillus burdens were impaired in the absence of TNF- $\alpha$ signaling.

(a) Experimental diagram for determining whether the effect of A. muciniphila depends on TNF- $\alpha$ signaling during M. tuberculosis infection. Antibiotics pretreated mice were gavaged with $A$. muciniphila $\left(2 \times 10^{8} \mathrm{CFU}\right)$ or saline for 3 times per week followed by aerosol M. tuberculosis infection. Mice were injected with $500 \mu \mathrm{g}$ anti-TNF- $\alpha$ MAb or IgG antibody control at the third day after M. tuberculosis infection and injections were performed every third day for a total of six injections.

(b) Gross pathology shows the hemorrhage and necrosis in lungs of M. tuberculosisinfected mice at 5 weeks post infection. Red circles mark the severe, unresolved hemorrhage, massive disruption or caseous necrosis on the lungs of $M$. tuberculosis-infected mice.

(c-d) Hematoxylin and eosin (H\&E) staining of four representative lungs (c) and the histological scores (d) of $M$. tuberculosis-infected mice at 5 weeks post infection. Top: original magnification; Middle: $2.5 \times$ of original magnification; Bottom: $10 \times$ of original magnification. The red-boxed areas at the top are enlarged below. Yellow arrowheads mark lesions and infiltration of inflammatory cells.

(e) Quantification analysis of $M$. tuberculosis CFU in the lung homogenates of $M$. tuberculosis-infected mice at 5 weeks post infection.

$\mathrm{N}=6$ mice per group. Error bars indicate SD. $P<0.05(*) ; P<0.01(* *) ; P<0.001$

$(* * *) ; P<0.0001(* * * *) ;$ NS (no statistical significance). $P$ values were calculated by 

biological repeats were performed. 


\section{Extended Data Fig. 6}
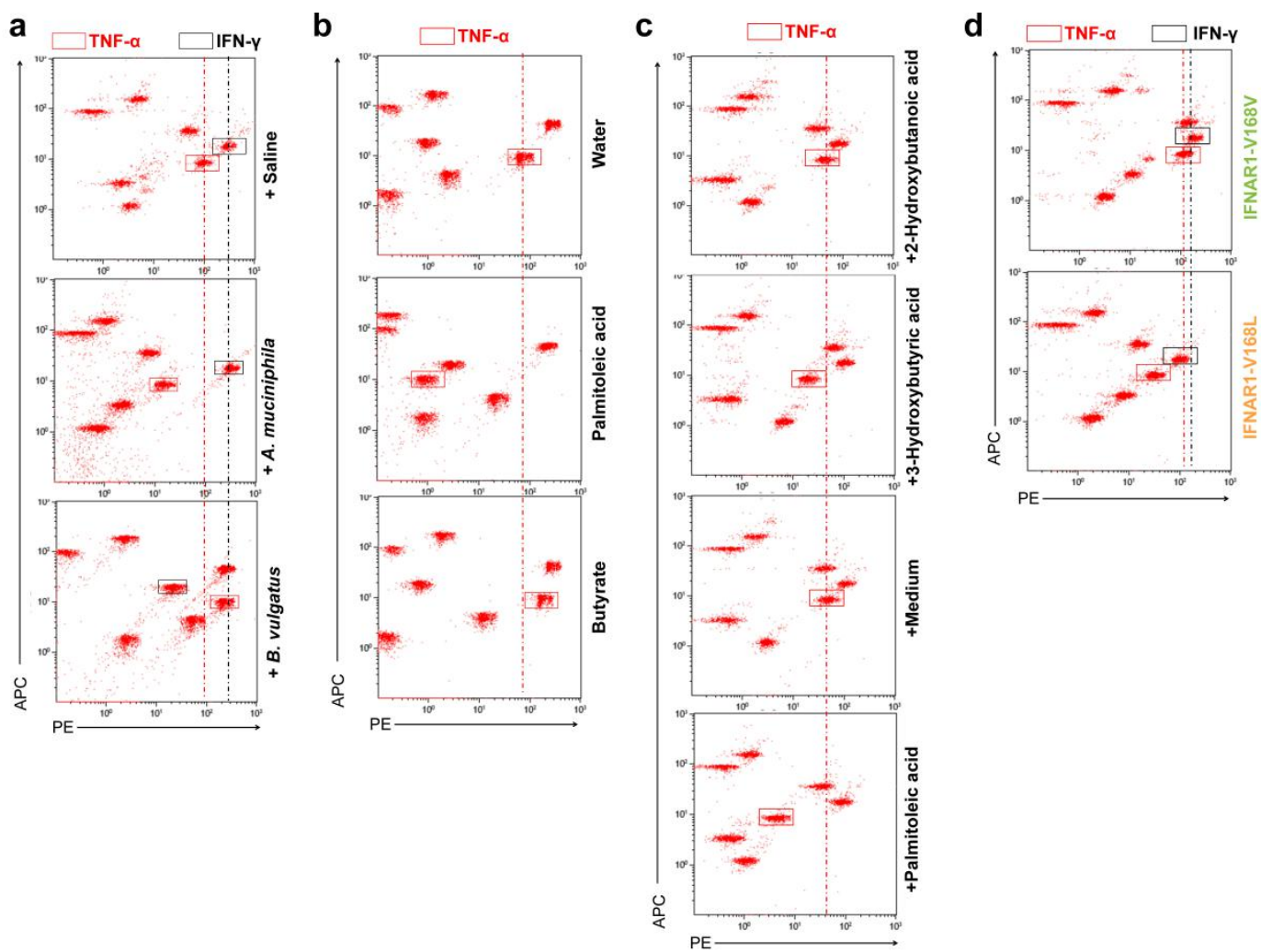

Extended Data Fig. 6. CBA analysis of cytokine productions in serum derived

from M. tuberculosis-infected mouse models.

(a) Representative CBA analysis of serum derived from M. tuberculosis-infected mice

(b) Representative CBA analysis of serum derived from M. tuberculosis-infected with oral administration of $A$. muciniphila, B. vulgatus and saline. Red and blackboxed areas mark the fluorescent clusters of TNF- $\alpha$ and IFN- $\gamma$, respectively, and dashed lines mark the shift of fluorescent clusters of TNF- $\alpha$ and IFN- $\gamma$, respectively. Pooled data were shown in Fig. 2i.

mice treated with palmitoleic acid, butyrate and water. Red and black-boxed areas mark the fluorescent clusters of TNF- $\alpha$, and dashed lines mark the shift of fluorescent clusters of TNF- $\alpha$. Pooled data were shown in Fig. 4g.

(c) Representative CBA analysis of culture supernatants of CD3+ T cells. CD3 $+\mathrm{T}$ 

$\mu \mathrm{g} / \mathrm{ml}$ ) only or with 2-Hydroxybutanoic acid, 3-Hydroxybutyric acid or palmitoleic acid for 3 days. Red-boxed areas mark the fluorescent clusters of TNF- $\alpha$, dashed line mark the shift of fluorescent clusters of TNF- $\alpha$. Pooled data were shown in Supplementary Fig. 3c.

(d) Representative CBA analysis of serum derived from $M$. tuberculosisinfectedIFNAR1-V168L and IFNAR1-V168V mice, respectively. Red and blackboxed areas mark the fluorescent clusters of TNF- $\alpha$ and IFN- $\gamma$, respectively, and dashed lines mark the shift of fluorescent clusters of TNF- $\alpha$ and IFN- $\gamma$, respectively. Pooled data were shown in Fig. 5p. 


\section{Extended Data Fig. 7}

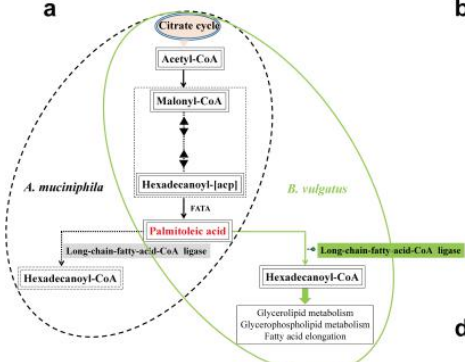

$\mathbf{f}$

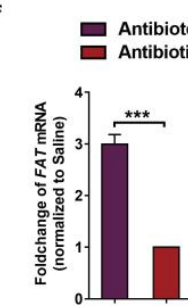

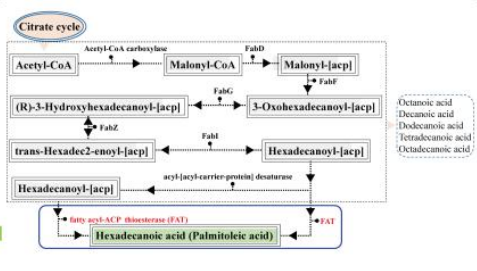

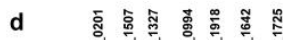
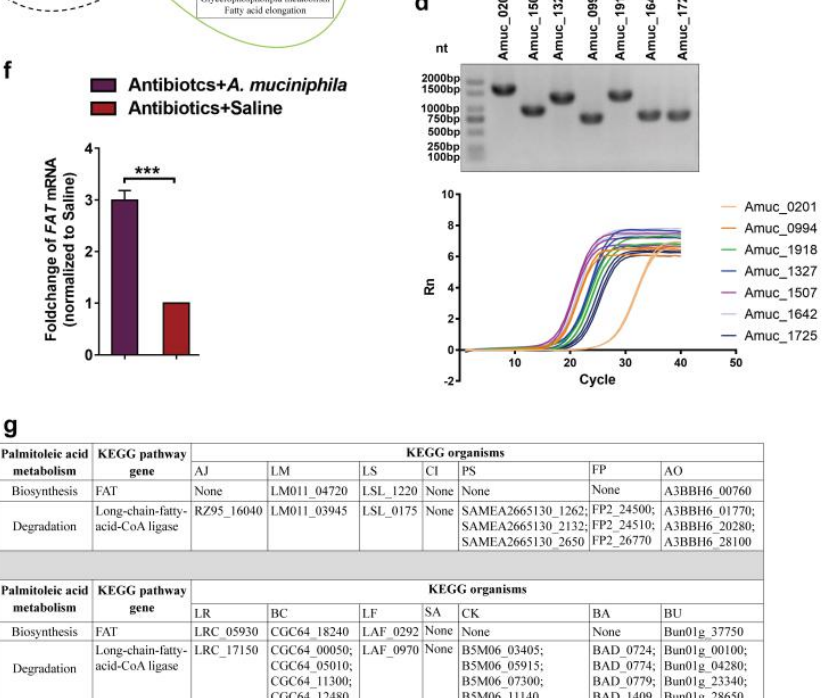

h

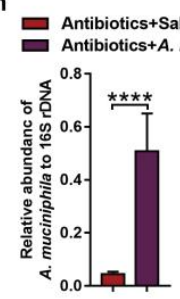

1265
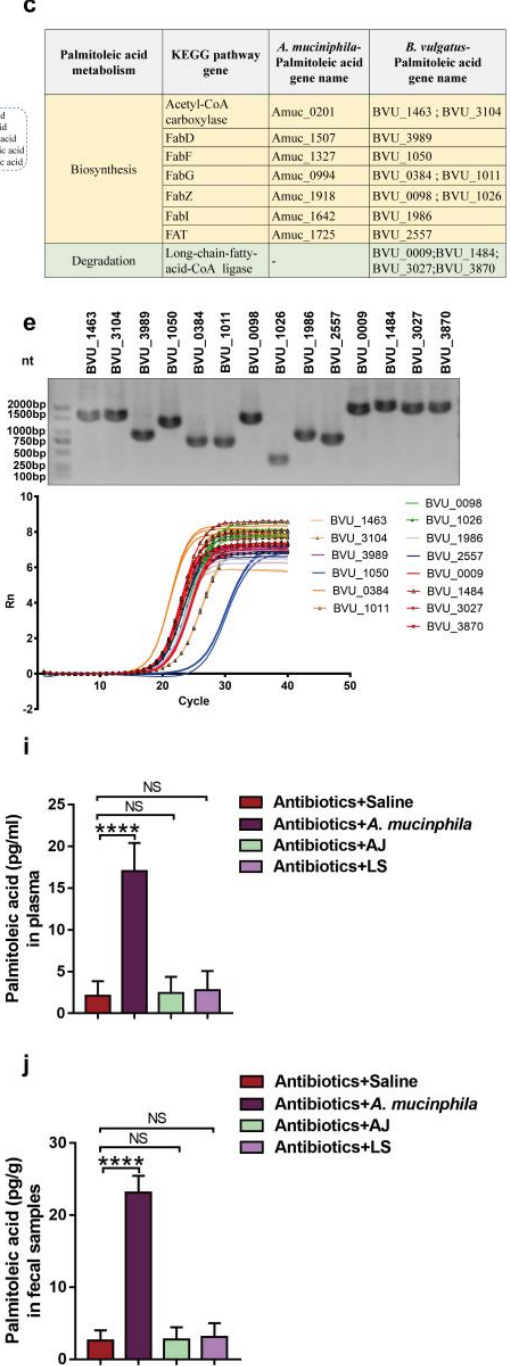

Extended Data Fig. 7. A. muciniphila played a dominant role in contributing to increased levels of palmitoleic acid in mice.

(a) Comparative KEGG pathway analyses of palmitoleic acid synthesis and degradation between $A$. muciniphila (marked with oval black dashed line) and $B$. vulgatus (marked with green solid line). The green box mark the presence of longchain-fatty-acid-CoA ligase for degrading/processing palmitoleic acid in $B$. vulgatus, and the gray box point the lack of presence (or at least lower level) of long-chain-fatty-acid-CoA ligase in A. muciniphila. The solid green arrow mark the downstream metabolic pathways for degrading/processing palmitoleic acid in 
B. vulgatus, and the black dashed arrow point that no (or at least less) long-chainfatty-acid-CoA ligase to degrade/process palmitoleic acid in A. muciniphila.

(b) Schematic diagram for palmitoleic acid biosynthesis pathway in A. muciniphila.

(f) The qPCR-based analysis of fatty acyl-ACP thioesterase (FAT) mRNA expression in fecal samples from M. tuberculosis-infected mice with A. muciniphila and saline treatment.

(g) Genes involved in palmitoleic acid metabolism pathway in gut bacteria, which showed significantly different abundance between $\mathrm{HC}$ and $\mathrm{TB}$. These bacteria include Acinetobacter johnsonii (AJ), Lactobacillus mucosae (LM), Lactobacillus salivarius (LS), Clostridium innocuum (CI), Plesiomonas shigelloides (PS), Comamonas kerstersii (CK), Alistipes onderdonkii (AO), Lactobacillus ruminis (LR), Streptococcus anginosus (SA), Lactobacillus fermentum (LF), Faecalibacterium prausnitzii (FP), Bifidobacterium adolescentis (BA), Bacteroides uniformis (BU) and Bacteroides caccae (BC).

(h) The qPCR-based quantitative analyses of $A$. muciniphila, A. johnsonii (AJ) and $L$. salivarius (LS) abundance in stool samples from antibiotics-treated mice with oral administration of $A$. muciniphila, AJ, LS or saline at the third day after 

bacteria gavage.

1298 (i-j) The quantitative analyses of palmitoleic acid concentrations in plasma (I) and fecal samples (J) derived from antibiotics-pretreated mice with oral 1300 administration of A. muciniphila, AJ, LS or saline at the third day after bacteria 1301 gavage.

$1302 \mathrm{~N}=6$ mice per group. Error bars indicate SD. $P<0.0001(* * * *) . P$ values were 1303 calculated by Student's two-tailed unpaired $t$-test $[(f),(h)]$ and one-way ANOVA with 1304 Tukey's multiple comparison test [(i) and (j)]. At least two biological repeats were 1305 performed. 


\section{Extended Data Fig. 8}

a

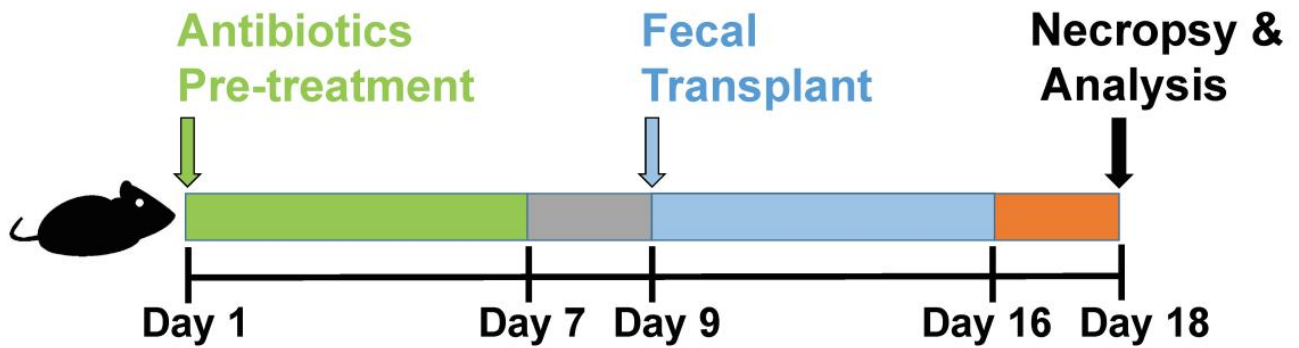

b

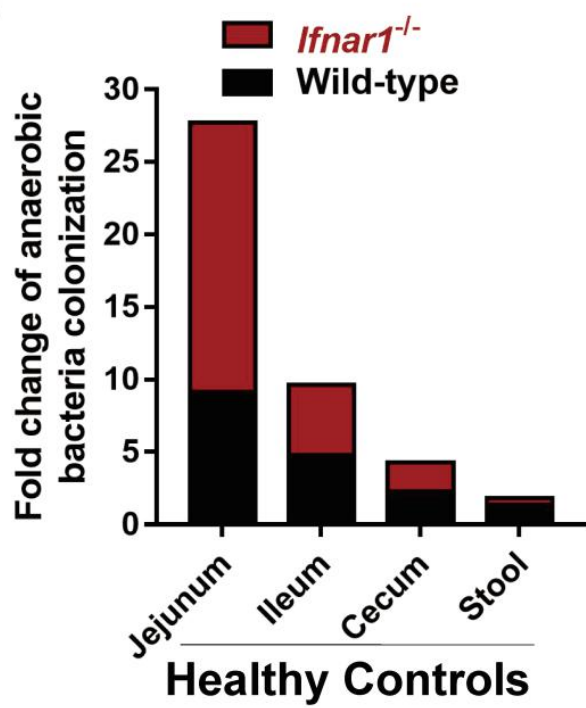

C

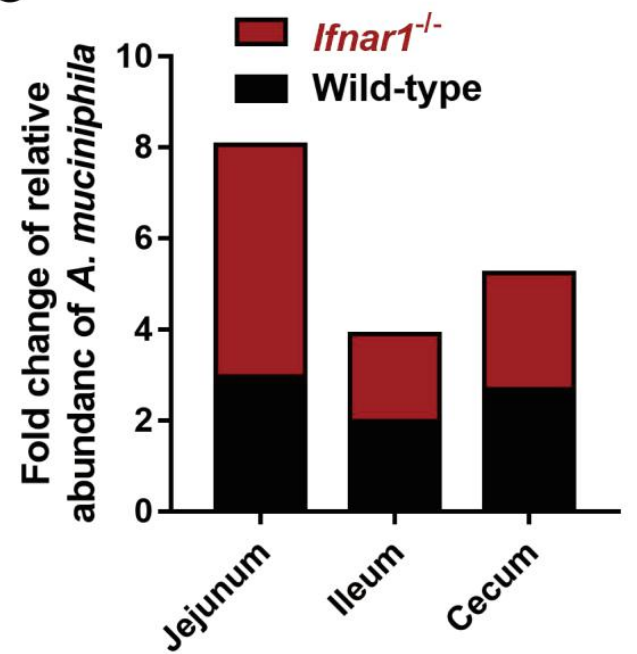

Healthy Controls
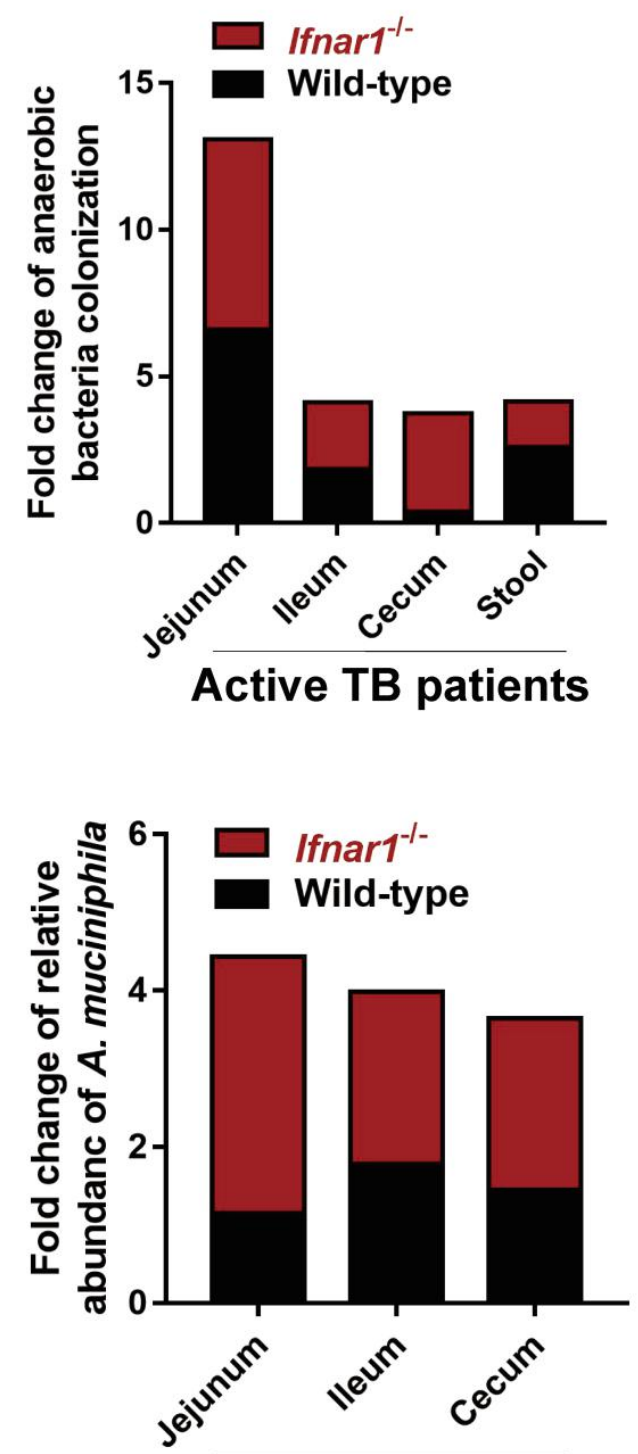

Active TB patients

Extended Data Fig. 8. Knock-out of an IFN-I receptor (Ifnar1) increases the

1308 intestinal colonization and abundance of bulk anaerobic bacteria and $A$. muciniphila. 
(a) Experiment design: Fecal microbiota transplantation (FMT) of fecal samples derived from $\mathrm{TB}$ and $\mathrm{HC}$ was performed following 7 days of antibiotics in Ifnar ${ }^{-}$ I- mice and wild-type mice. Eighteen days later, the anaerobic bacteria as well as $A$. muciniphila in jejunum, ileum, cecum and fecal samples were analyzed.

(b) Pooled data of relative amounts of anaerobic bacteria recovered from jejunum, ileum or cecum of Ifnarl $^{-/-}$mice (red bar) and wild-type mice (black bar), respectively. Mice were administrated with fecal samples of HC (Left panel) or TB (Right panel) as described in (a), and then tissues of jejunum, ileum or cecum and fecal samples of mice were subjected to anaerobic culture, followed by quantitative analysis of anaerobic bacteria CFU. Fold changes were calculated as $\mathrm{CFU}$ of anaerobic bacteria recovered from jejunum, ileum or cecum and fecal samples of mice administrated with fecal samples of $\mathrm{HC}$ or TB normalized to those of saline-treated Ifnar $1^{-/-}$mice and wild-type mice. $\mathrm{N}=6$ mice per group.

(c) Pooled data of relative gene expression levels of $A$. muciniphila recovered from jejunum, ileum, or cecum of Ifnar $^{-/-}$mice (red bar) and wild-type mice (black bar), respectively. Mice were administrated with fecal samples of HC (Left panel) or TB (Right panel) as described in (a), and then tissues of jejunum, ileum, or cecum were subjected to qPCR analysis of DNA extracted from jejunum, ileum, and cecum of mice. Fold changes were calculated as gene expression levels of $A$. muciniphila recovered from jejunum, ileum, or cecum of mice administrated with fecal samples of $\mathrm{HC}$ or $\mathrm{TB}$ normalized to those of same genotype mice with oral saline administration. $\mathrm{N}=6$ mice per group. 
Extended Data Fig. 9

a
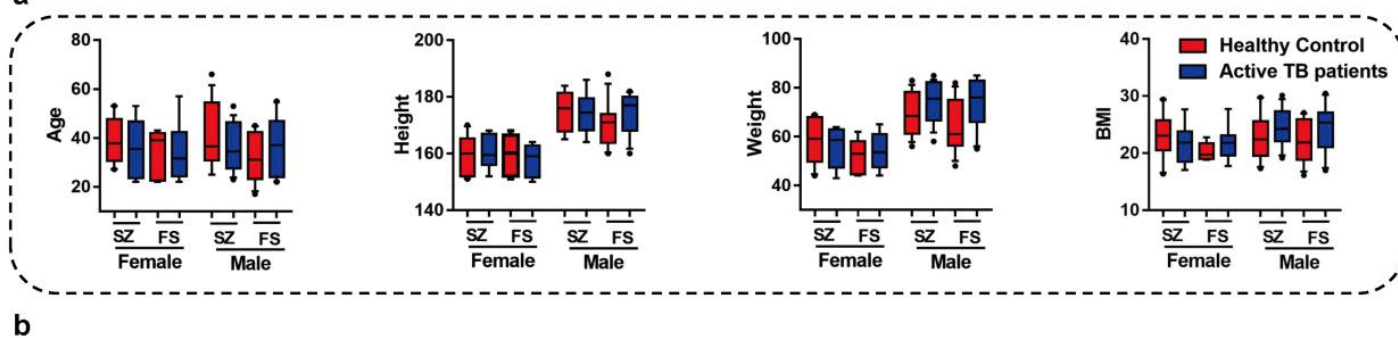

b
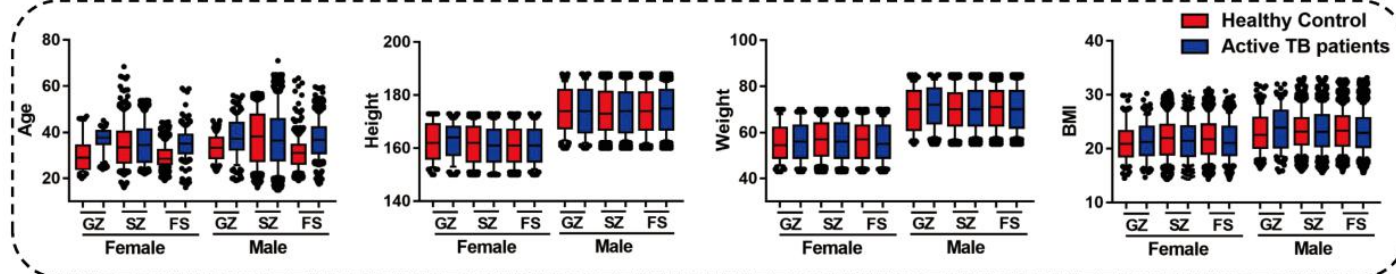

c

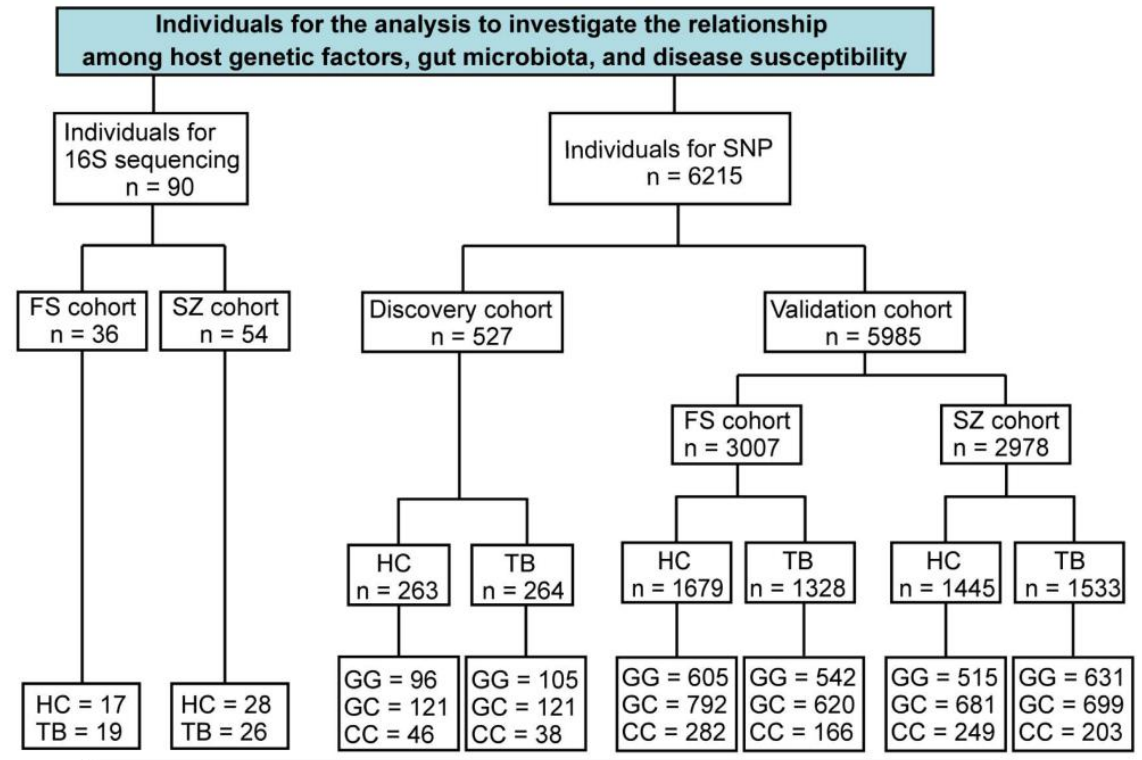

A. muciniphila abundance, metabolites in fecal and plasma

cytokine (TNF- $\alpha$ ) analysis, human Ifnar1 SNP, HRCT score of TB pathology

\section{3}

Extended Data Fig. 9. Boxplot and flow chart illustration of characteristics of

\section{Shenzhen, Foshan and Guangzhou cohorts.}

(a) Boxplot illustrating the distribution of age, height, weight, and body mass index (BMI) of individuals for analyses of $16 \mathrm{~S}$ rDNA in Shenzhen $(\mathrm{HC}=28, \mathrm{~TB}=26)$ and Foshan $(\mathrm{HC}=17, \mathrm{~TB}=19)$ cohorts. SZ: Shenzhen cohort. FS: Foshan cohort.

(b) Boxplot illustrating the distribution of age, height, weight, and body mass index (BMI) of individuals for SNP analyses in Guangzhou $(\mathrm{HC}=263, \mathrm{~TB}=264)$, 

GZ: Guangzhou cohort. SZ: Shenzhen cohort. FS: Foshan cohort.

(c) Flow chart for each cohort. Flow chart to illustrate sample collection and analyses

1343 that were undertaken with them.

1344 


\section{Extended Data Fig. 10}

\section{a}

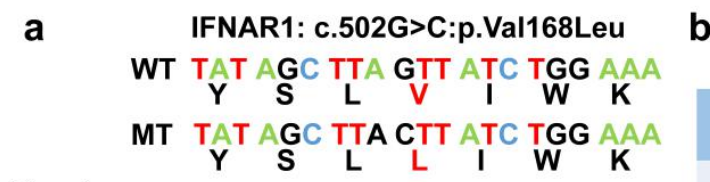

Genotype

\begin{tabular}{|c|c|c|c|}
\hline SNP ID & Location & $\begin{array}{c}\text { Allele } \\
\text { change }\end{array}$ & $\begin{array}{c}\text { Amino acid } \\
\text { change }\end{array}$ \\
\hline rs2257167 & $\begin{array}{l}\text { Ifnar1 } \\
\text { Exon 4 }\end{array}$ & G>C & V168L \\
\hline
\end{tabular}
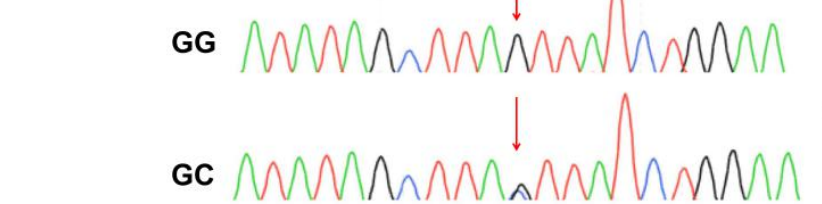

C
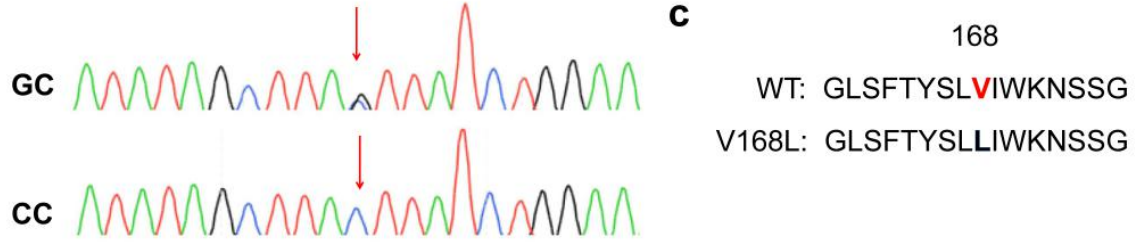

V168L: GLSFTYSLLIWKNSSG

Extended Data Fig. 10. Identified rs2257167 variant in Ifnar1 gene leads to a

V168L variant.

(a) DNA sequencing data of three men with the different genotype of rs2257167 in Ifnarl gene. WT, wild-type; MT, mutant.

(b) SNP locus rs2257167 in human Ifnarl gene.

1351

(c) Comparison of the wild-type and mutated element in human IFNAR1 protein. 
Extended Data Fig. 11
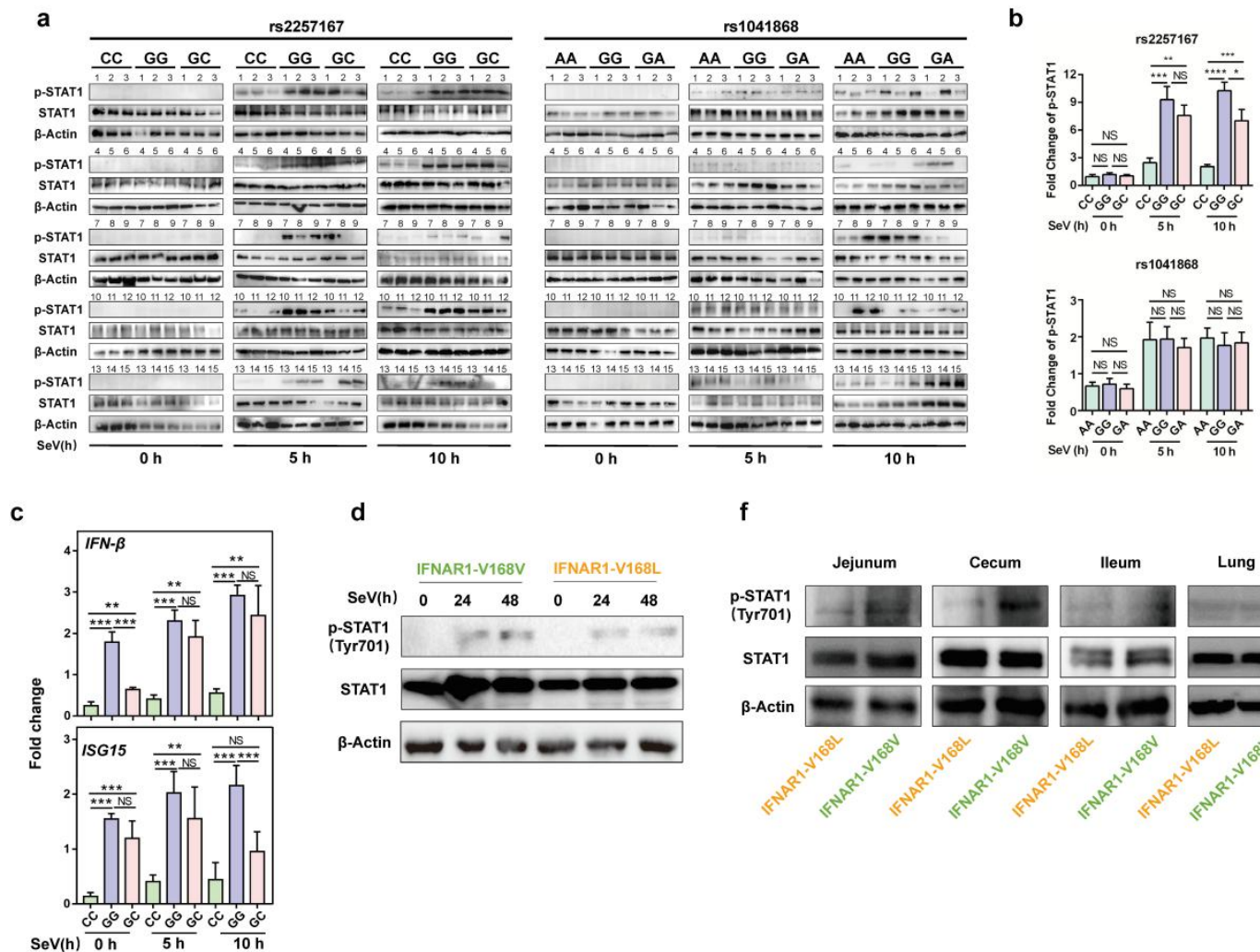

d
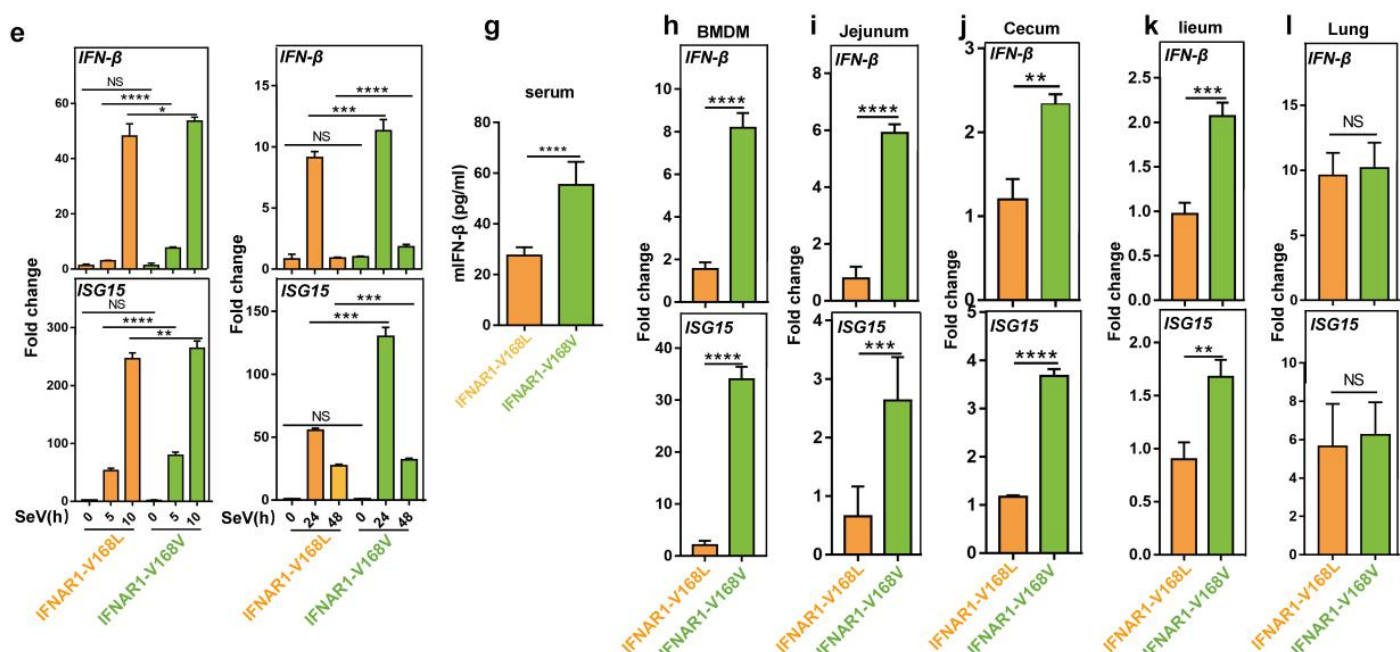

Extended Data Fig. 11. Rs2257167 G allele in Ifnar1 gene enhances IFN-I signaling in both humans and mice.

(a) Immunoblot analysis of the levels of phosphorylated(p)- and total STAT1 in PBMCs. Fifteen individuals of each genotype were examined (individual numbers for rs2257167, $\mathrm{GG}=15, \mathrm{GC}=15, \mathrm{CC}=15$; individual numbers for rs1041868, 
$\mathrm{GG}=15, \mathrm{GA}=15, \mathrm{AA}=15)$. Patient number is shown above in each of blot lane.

(b) Quantitative immunoblot analysis of expression of p-STAT1. ImageJ was used for quantitative analysis of immunoblot.

(c) The qRT-PCR-based mRNA expression of $I F N-\beta$ and $I S G 15$ in PBMCs derived from individuals carrying genotype $\mathrm{GG}, \mathrm{GC}, \mathrm{CC}$ at 0,5 and 10 hours after stimulation with Sendai virus. Data are representative of three experiments with three independent biological replicates.

(d) Immunoblot analysis of the expression levels of p- and total STAT1 in BMDM derived from IFNAR1-V168V and IFNAR1-V168L mice at 0, 24 and 48 hours following Sendai virus stimulation. Data are representative of three experiments with three independent biological replicates.

(e) The qRT-PCR-based analysis of mRNA expression of IFN- $\beta$ and ISG15 in BMDM derived from IFNAR1-V168V and IFNAR1-V168L mice at 0, 5, 10, 24 and 48 hours following Sendai virus stimulation. $\mathrm{N}=6$ mice per group.

(f) Immunoblot analysis of the expression levels of p- and total STAT1 in jejunum, ileum,cecum and lung derived from IFNAR1-V168V and IFNAR1-V168L mice at 7 days following M. tuberculosis infection. Data are representative of three experiments with three independent biological replicates.

(g) ELISA analysis of IFN- $\beta$ concentrations in serum derived from IFNAR1-V168V and IFNAR1-V168L mice at 7th day following M. tuberculosis infection. $\mathrm{N}=6$ mice per group.

(h-l) The mRNA expression of IFN- $\beta$ and ISG15 in tissues including BMDM (h), 
1380 jejunum (i), cecum (j),ileum (k) and lung (1) derived from IFNAR1-V168V and 1381 IFNAR1-V168L mice, respectively, at 7th day following M. tuberculosis infection. $1382 \quad \mathrm{~N}=6$ mice per group.

1383 Error bars indicate SD. $P<0.01\left(^{* *}\right) ; P<0.001(* * *) ; P<0.0001\left(^{* * * *}\right.$ ); NS (no 1384 statistical significance). $P$ values were calculated by one-way ANOVA with Tukey's 1385 multiple comparison test [(b), (c)], Student's two-tailed unpaired $t$-test [(e), (g) to (l)]. 1386 At least two biological repeats were performed. 


\section{Extended Data Fig. 12}

a

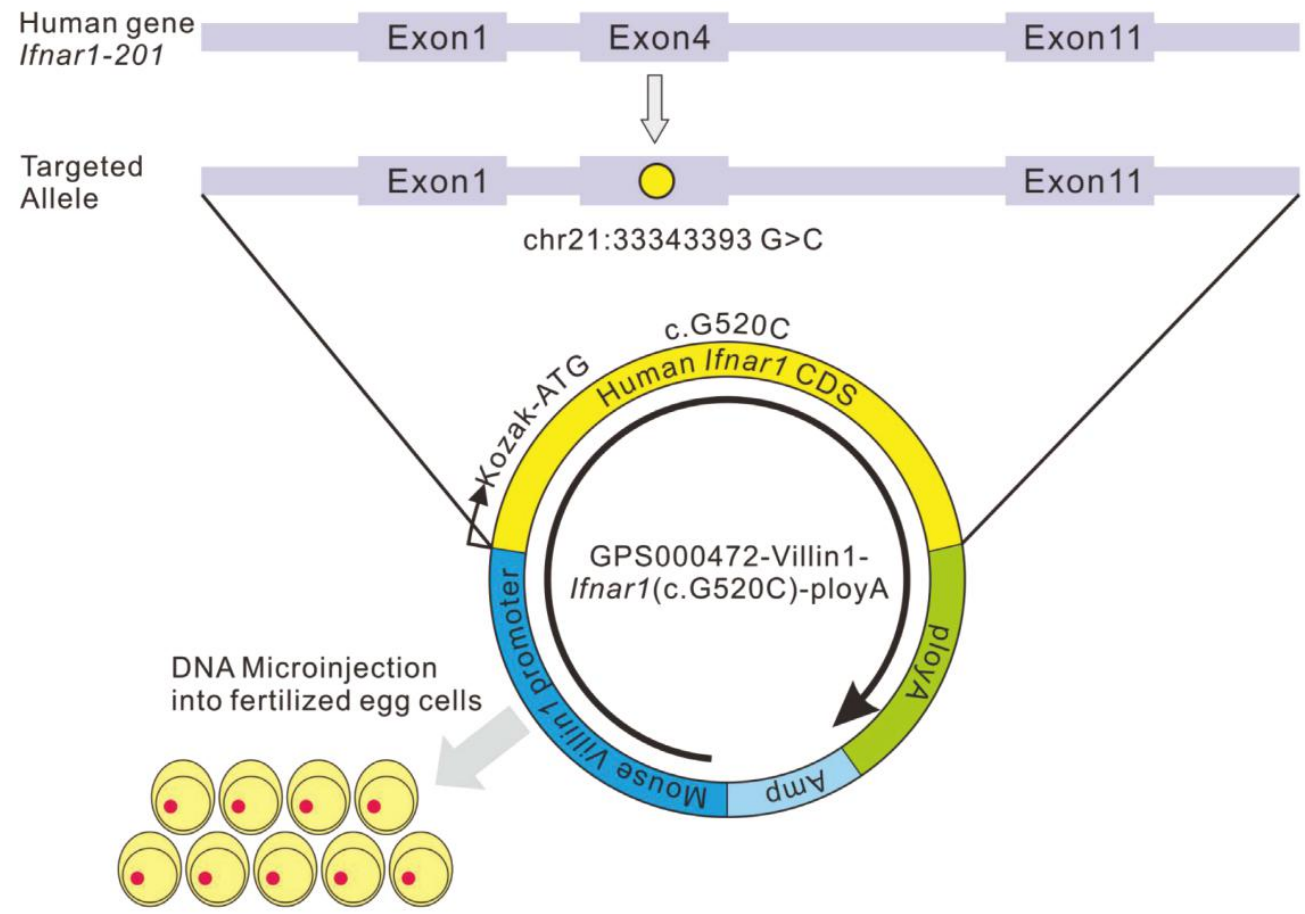

Fertilized egg transfer

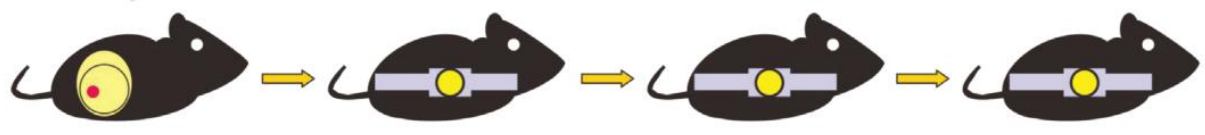

Surrogant mother mice

F0

F1

F2

b
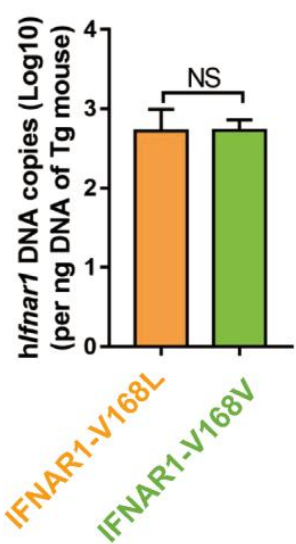

C
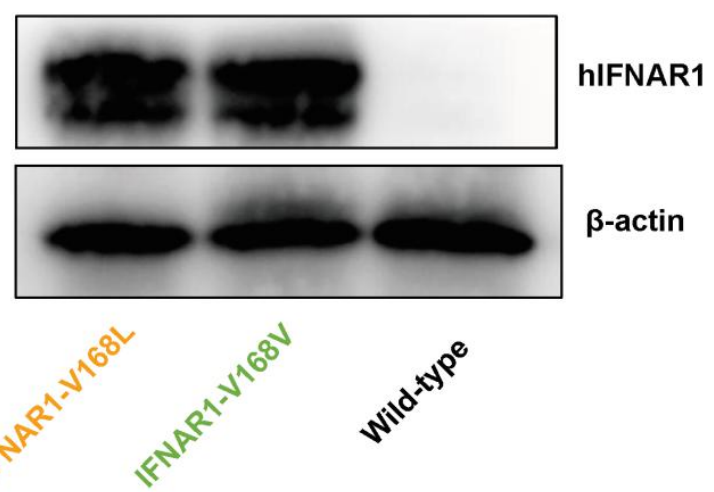

Extended Data Fig. 12. Construction strategy of transgenic mice with the human Ifnar1 rs2257167 $\mathrm{G}$ allele or $\mathrm{C}$ allele.

(a) Ifnar1-201(ENST00000270139.7) was selected for construction of transgenic 
mice. The 520th base of the fourth exon of the human Ifnarl gene was changed from $\mathrm{G}$ to $\mathrm{C}$, The resulting human Ifnarl was ligated to the mouse epithelium promoter, villinl as regulatory element. The transgenic fragment was obtained: mouse- Kozak-villin1-human Ifnarl (c.G520C) CDS. The DNA fragments were randomly integrated into the mouse genome by microinjection technology, and the born mice were genetically identified by PCR to obtain F0 generation mice with the target DNA. Ifnarl-201(ENST00000270139.7) has 11 coding exons, transcript length of $6,139 \mathrm{bp}$ and translation length of 557 residues. Kozak sequence (GCCGCCACC) was added before the ATG, which allowed the ribosome to

1401 (b) Absolute quantitation analysis of hIfnarl copies in transgenic mice. $\mathrm{N}=6$ mice per group.

(c) Representative western blot showed that there was no significant difference in V168V transgenic mice. $\mathrm{N}=6$ mice per group. unpaired $t$-test. 


\section{Extended Data Fig. 13}

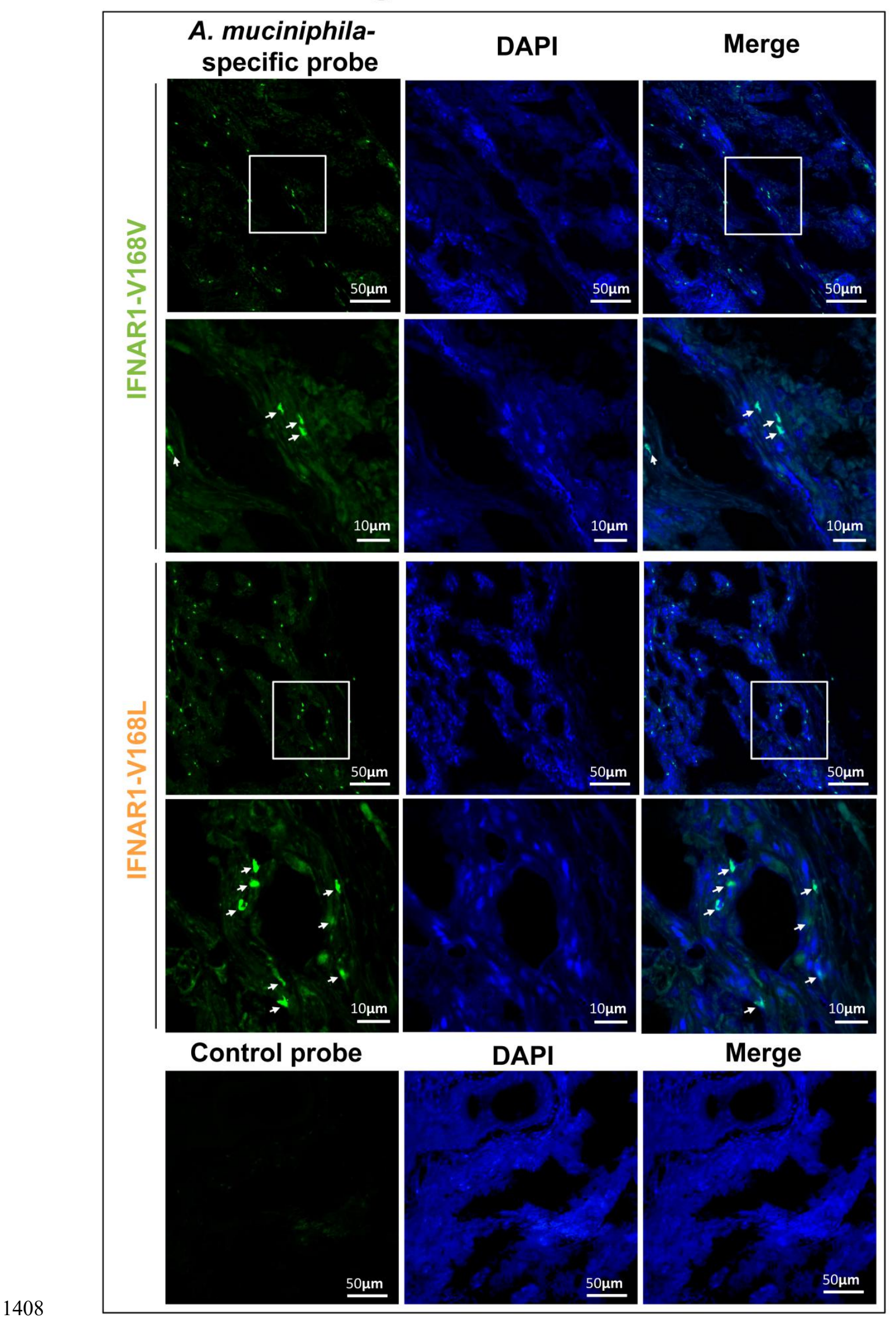

1409 Extended Data Fig. 13. Confocal microscopic imaging shows that IFNAR1- 
1411 V168L mice.

1412 Representative fluorescent in situ hybridization (FISH)/confocal microscopic imaging

1413 analysis of $A$. muciniphila (green) on the intestinal mucosa by using an $A$. 1414 muciniphila-specific probe. DAPI (4', 6-diamidino-2-phenylindole) was used for 1415 nuclear staining (blue). The white-boxed areas at the upper panel images are enlarged 1416 in the lower panel. The upper images were obtained at $20 \times$ magnification, the lower 1417 images were obtained at $60 \times$ magnification. Scale bars, $50 \mu \mathrm{m}$ (Top) and $10 \mu \mathrm{m}$ 1418 (Bottom). The white arrowheads mark the A. muciniphila. 


\section{Extended Data Fig. 14}

a

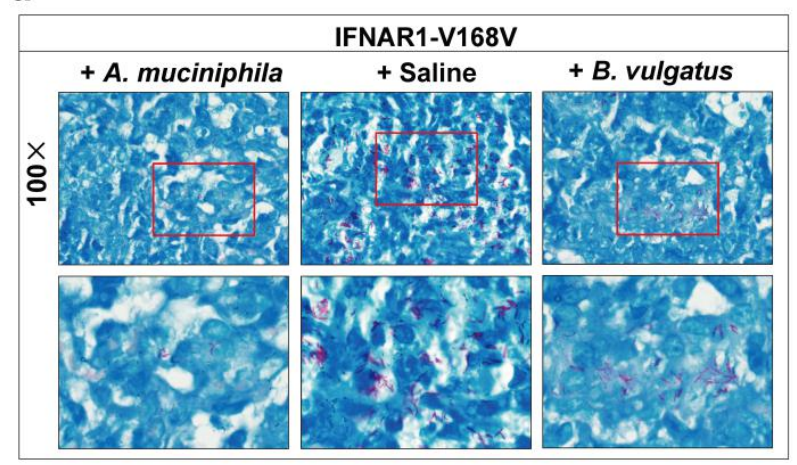

b

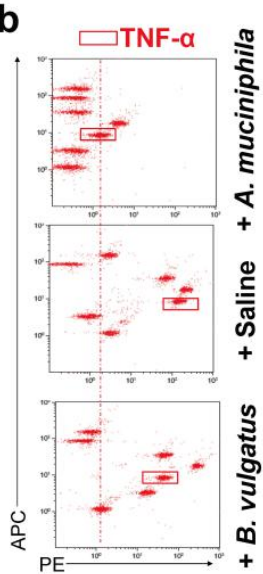

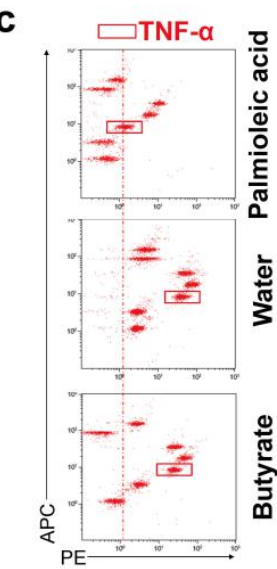

1420

1421

1422

1423

1424

1425

1426

1427

1428

1429

1430

1431

1432

1433

1434

1435

Extended Data Fig. 14. Oral gavage of $A$. muciniphila or palmitoleic acid reduces

bacillus burdens and TNF- $\alpha$ production in M. tuberculosis-infected IFNAR1-

V168V mice.

(a) The acid-fast staining to visualize $M$. tuberculosis in lung sections at 5 weeks post infection. Note that less acid-fast staining-positive bacilli in lung sections derived from A. muciniphila-treated IFNAR1-V168V mice. Top: $100 \times$ of original magnification. The red-boxed areas at the top are enlarged below.

(b) Representative CBA analysis of serum derived from M. tuberculosis-infected IFNAR1-V168V mice with oral administration of $A$. muciniphila, B. vulgatus and saline. Red-boxed areas mark the fluorescent clusters of TNF- $\alpha$ and dashed lines mark the shift of fluorescent clusters of TNF- $\alpha$.

(c) Representative CBA analysis of serum derived from M. tuberculosis-infected IFNAR1-V168V mice with dietary palmitoleic acid, butyrate or water. Red-box mark the fluorescent clusters of TNF- $\alpha$ and dashed lines mark the shift of fluorescent clusters of TNF- $\alpha$. 


\section{Extended Data Fig. 15}
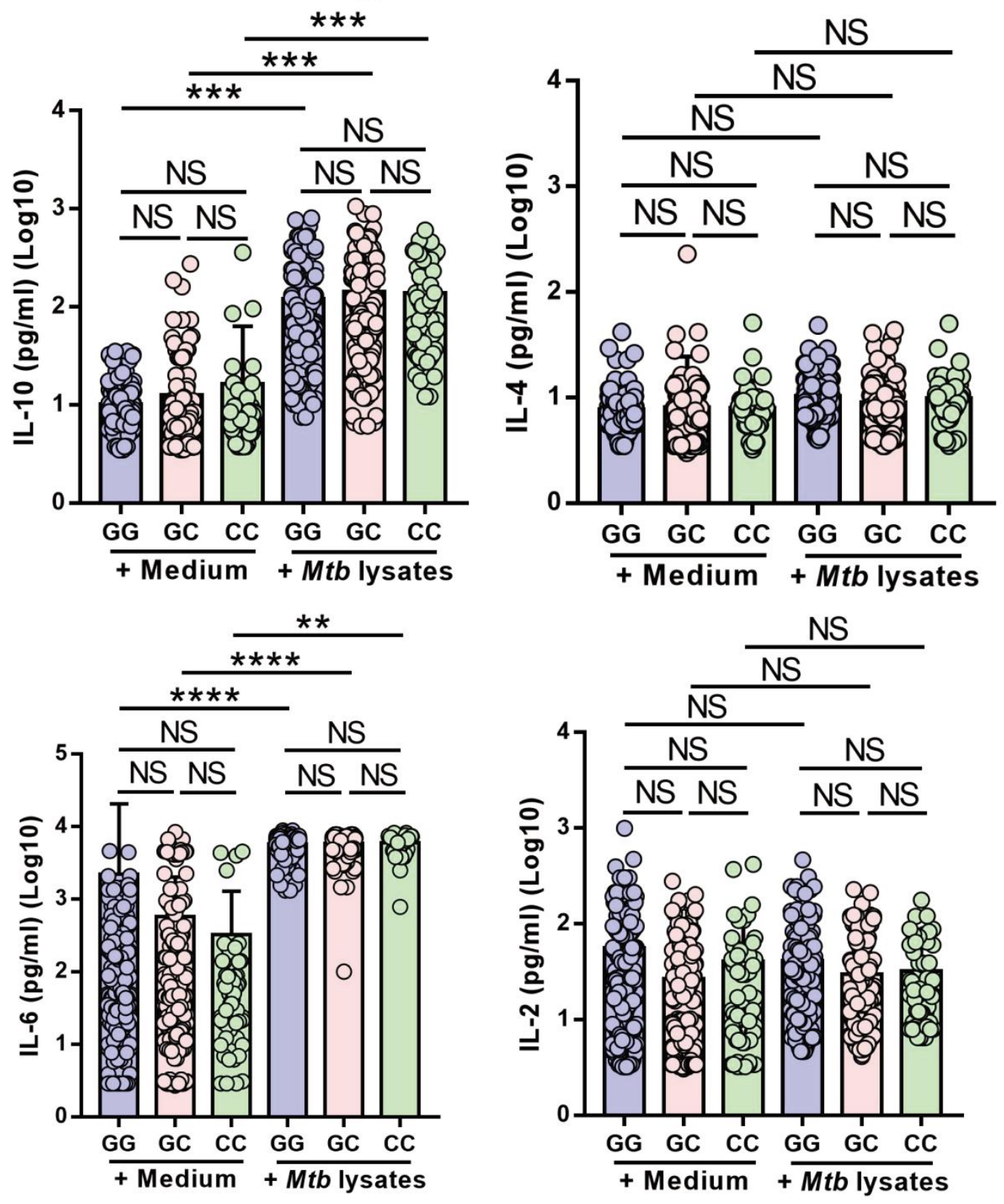

Extended Data Fig. 15. Pooled bar graphic data shows the expression of IL-10,

IL-6, IL-2, and IL-4 in culture supernatants of PBMCs derived from TB patients

1439 carrying genotype GG, GC, CC.

1440 PBMCs derived from TB patients carrying genotype GG $(n=185)$, GC $(n=206)$ or

$1441 \mathrm{CC}(\mathrm{n}=62)$ were ex vivo stimulated with $M$. tuberculosis lysates and analyzed as in $1442 \quad$ Fig. 7. 
1443 Error bars indicate SD. $P<0.01(* *) ; P<0.001(* * *) ; P<0.0001\left(^{* * * *}\right)$; NS (no 1444 statistical significance). $P$ values were calculated by one-way ANOVA with 1445 Newman-Keuls multiple comparison test.

1446 


\section{Extended Data Fig. 16}

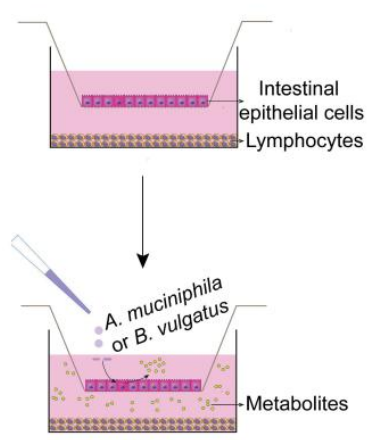

d

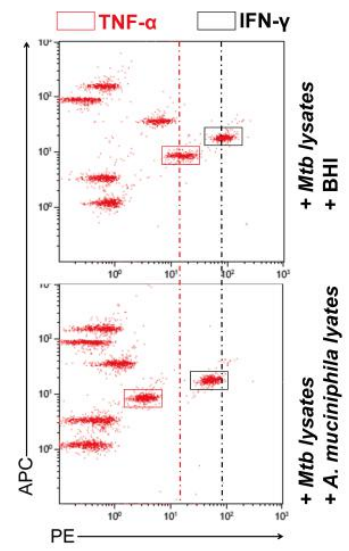

g

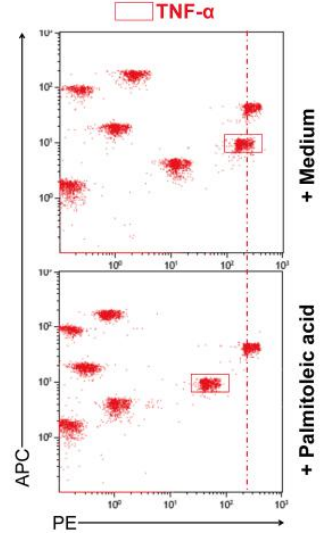

b

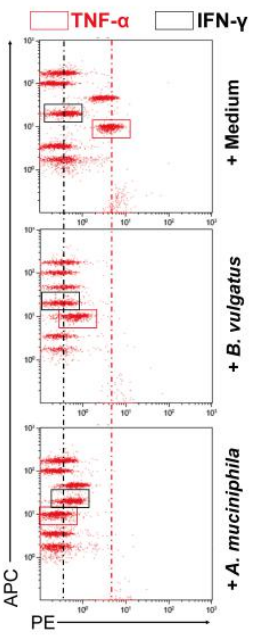

e $\square+$ Mtb lysates

+ Mtb lysates

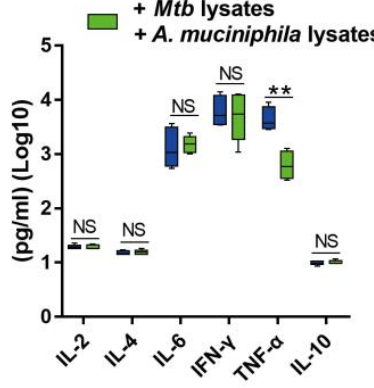

h

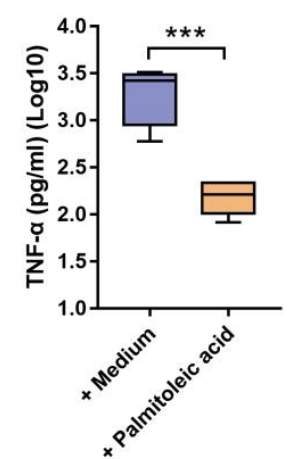

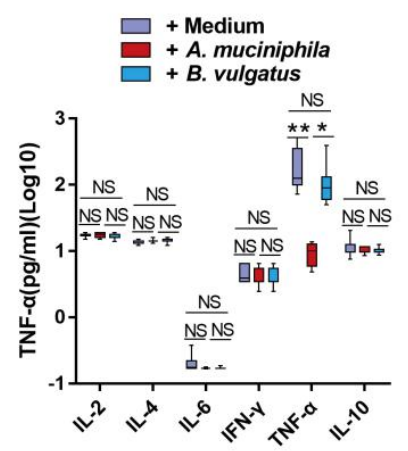

f

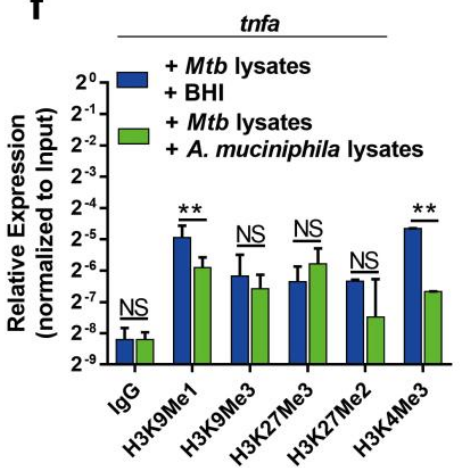

i

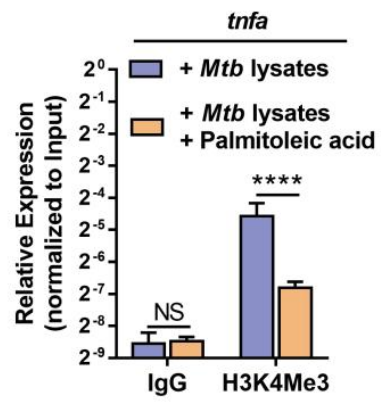

Extended Data Fig. 16. Intestine-colonized $A$. muciniphila and an $A$. muciniphiladerived metabolite palmitoleic acid, inhibit $M$. tuberculosis-specific TNF-a expression via an epigenetic mechanism.

(a) The experimental diagram of transwell assay to mimic the effects of epitheliumcolonized gut bacteria on cytokine production. intestinal epithelial cells $\left(2 \times 10^{5}\right)$ were seeded into the upper chamber, $\mathrm{CD} 3+\mathrm{T}$ cells isolated from autologous 
spleens $\left(2 \times 10^{5}\right)$ were seeded to the lower chamber, and A. muciniphila $\left(1 \times 10^{5}\right.$ CFU) or B. vulgatus $\left(1 \times 10^{5} \mathrm{CFU}\right)$ were added to the upper chamber. After 12 hours incubation, culture supernatants in the lower chamber were detected for the expression of cytokines.

(b) Representative CBA analysis of culture supernatants of CD3+ T cells in the lower

(c) Pooled bar graphic data show the expression of TNF- $\alpha /$ IFN- $\gamma / \mathrm{IL}-2 / \mathrm{IL}-4 / \mathrm{IL}-6 / \mathrm{IL}-$ 10 in culture supernatants of $\mathrm{CD} 3+\mathrm{T}$ cells in the lower chamber. $\mathrm{CD} 3+\mathrm{T}$ cells were cultured with medium only or with $A$. muciniphila $\left(1 \times 10^{5} \mathrm{CFU}\right)(+A$. muciniphila $)$ and B. vulgatus $\left(1 \times 10^{5} \mathrm{CFU}\right)(+$ B. vulgatus $)$ for 12 hours. $\mathrm{N}=7$ mice per group.

(d) Representative CBA analysis of culture supernatants of CD3+ T cells of $M$. tuberculosis-infected mice. CD3+ T cells were co-cultured with A. muciniphila lysates $(10 \mu \mathrm{g} / \mathrm{ml})$ or BHI in presence of $M$. tuberculosis lysates $(10 \mu \mathrm{g} / \mathrm{ml})$. Red and black-boxed areas mark the fluorescent clusters of TNF- $\alpha$ and IFN- $\gamma$, respectively, and dashed line mark the shift of fluorescent clusters of TNF- $\alpha$ and IFN- $\gamma$, respectively.

(e) Pooled bar graphic data show the in vitro expression of TNF- $\alpha /$ IFN- $\gamma / \mathrm{IL}-2 / \mathrm{IL}-$ 4/IL-6/IL-10 in culture supernatants of CD3+ T cells. $\mathrm{T}$ cells were co-cultured with $A$. muciniphila lysates $(10 \mu \mathrm{g} / \mathrm{ml})$ or BHI in presence of $M$. tuberculosis 
lysates $(10 \mu \mathrm{g} / \mathrm{ml})$ for 3 days. $\mathrm{N}=7$ mice per group.

(f) CHIP-qPCR analysis of IgG, H3K4Me3, H3K9Me1, H3K9Me3, H3K27Me2 and $\mathrm{H} 3 \mathrm{~K} 27 \mathrm{Me} 3$, and control antibodies at the promoter of thf $\alpha . \mathrm{CD} 3+\mathrm{T}$ cells were randomly selected for co-culturing with A. muciniphila lysates $(10 \mu \mathrm{g} / \mathrm{ml})$ or BHI in presence of $M$. tuberculosis lysates $(10 \mu \mathrm{g} / \mathrm{ml})$ for 3 days.

(g) Representative CBA analysis of culture supernatants of CD3+ T cells. CD3 $+\mathrm{T}$ cells derived from spleen of mice were cultured with $M$. tuberculosis lysates (10 $\mu \mathrm{g} / \mathrm{ml}$ ) only or with $A$. muciniphila-derived metabolite, palmitoleic acid for 3 days Red-boxed areas mark the fluorescent clusters of TNF- $\alpha$, dashed line mark the shift of fluorescent clusters of TNF- $\alpha$.

(h) Pooled bar graphic data show the expression of TNF- $\alpha$ in culture supernatants of $\mathrm{CD} 3+\mathrm{T}$ cells of wild-type mice. $\mathrm{CD} 3+\mathrm{T}$ lymphocytes isolated from spleens were cultured with palmitoleic acid $(10 \mu \mathrm{M})$ plus M. tuberculosis lysates $(10 \mu \mathrm{g} / \mathrm{ml})$ or with $M$. tuberculosis lysates $(10 \mu \mathrm{g} / \mathrm{ml})$ only. $\mathrm{N}=7$ mice per group.

(i) $\mathrm{CHIP}-\mathrm{qPCR}$ analysis of $\mathrm{H} 3 \mathrm{~K} 4 \mathrm{Me} 3$ and $\mathrm{IgG}$ control at thf $\alpha$ promoter. $\mathrm{CD} 3+\mathrm{T}$ cells from mouse spleens were cultured with $M$. tuberculosis lysates $(10 \mu \mathrm{g} / \mathrm{ml})$ only or with palmitoleic acid $(10 \mu \mathrm{M})$ plus $M$. tuberculosis lysates $(10 \mu \mathrm{g} / \mathrm{ml})$.

Error bars indicate SD. $P<0.01(* *) ; P<0.001(* * *) ; P<0.0001(* * * *)$; NS (no statistical significance). $P$ values were calculated by one-way ANOVA with Tukey's multiple comparison test (c) and Student's two-tailed unpaired $t$-test [(e), (f), (h) and (i)]. At least two biological repeats were performed. 
Extended Data Fig. 17
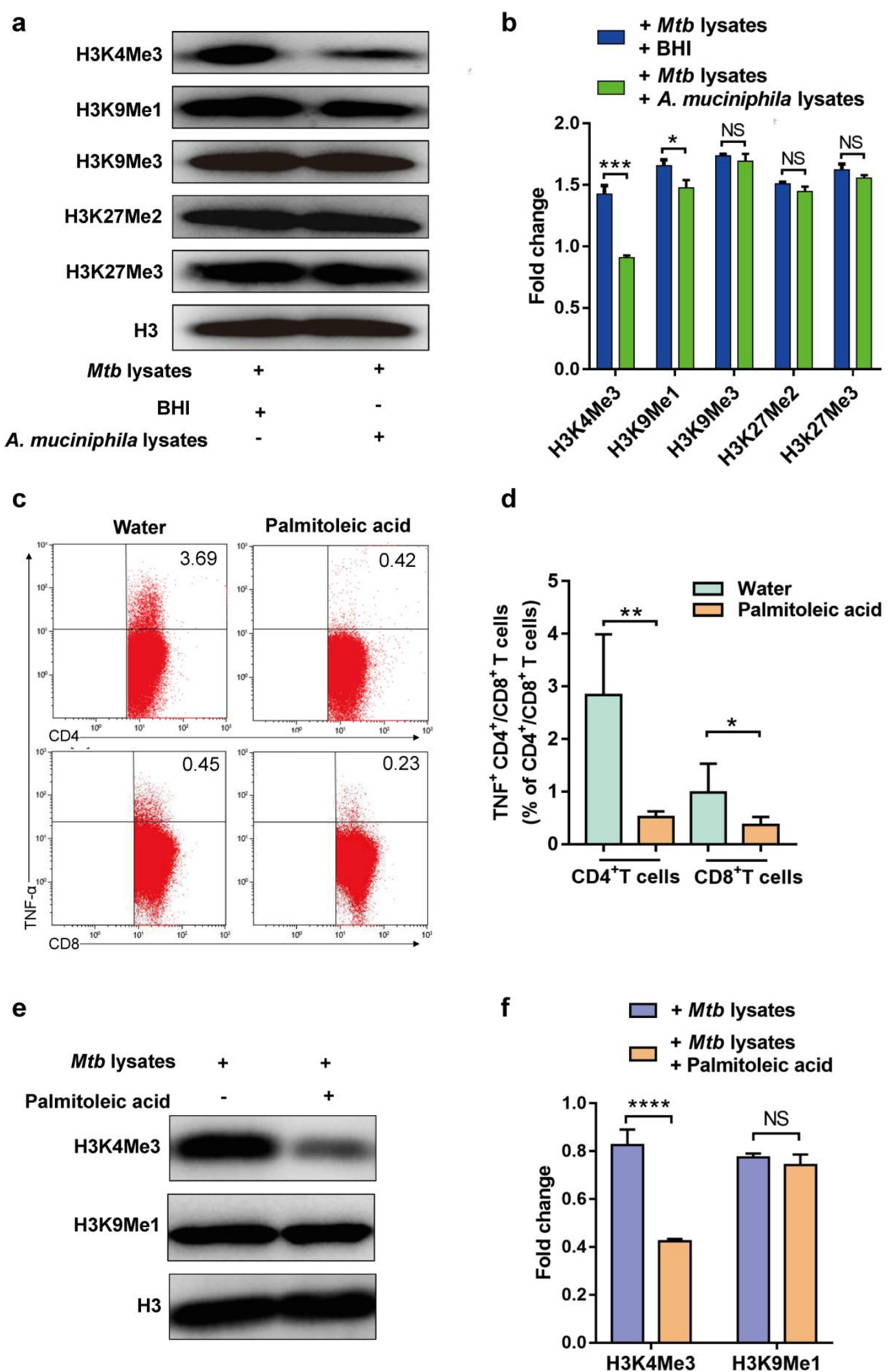

Extended Data Fig. 17. A. muciniphila uses its metabolite, palmitoleic acid, to 


\section{reduce H3K4Me3 expression.}

(a) Immunoblot analysis of H3K4Me3, H3K9Me1, H3K9Me3, H3K27Me2 and H3K27Me3 in CD3+ T cells co-cultured with A. muciniphila lysates $(10 \mu \mathrm{g} / \mathrm{ml})$ or BHI in presence of $M$. tuberculosis lysates $(10 \mu \mathrm{g} / \mathrm{ml})$ for 3 days.

(b) Quantitative immunoblot analysis of expression of H3K4Me3, H3K9Me1, H3K9Me3, H3K27Me2 and H3K27Me3. ImageJ was used for quantitative analysis of immunoblot.

(c-d) Representative flow cytometric dot plots and pooled bar graphic data show the expression of TNF- $\alpha$ on CD4+ (CD8+) $\mathrm{T}$ cells in spleens derived from $M$. tuberculosis-infected mice with dietary treatments of palmitoleic acid, butyrate or water at 5 weeks post infection. $\mathrm{N}=6$ mice per group.

(e) Immunoblot analysis of $\mathrm{H} 3 \mathrm{~K} 4 \mathrm{Me} 3$ and $\mathrm{H} 3 \mathrm{~K} 9 \mathrm{Me}$ of CD3 + T cells cultured with $M$. tuberculosis lysates $(10 \mu \mathrm{g} / \mathrm{ml})$ only or with A. muciniphila-derived metabolite, palmitoleic acid.

(f) Quantitative immunoblot analysis of H3K4Me3 and H3K9Me1 by imageJ.

Error bars indicate SD. $P<0.05(*) ; P<0.01(* *) ; P<0.001(* * *) ; P<0.0001$ (****); NS (no statistical significance). $P$ values were calculated by Student's twotailed unpaired $t$-test [(b), (d) and (f)]. At least two biological repeats were performed. 


\begin{tabular}{|c|c|c|c|c|c|c|c|c|}
\hline Cohort & Groups & $\begin{array}{c}\text { Sample } \\
\text { size }\end{array}$ & Sex & Number & Age & Height & Weight & BMI \\
\hline \multirow{4}{*}{$\begin{array}{l}\text { Shenzhen } \\
\text { cohort }\end{array}$} & \multirow{2}{*}{$\begin{array}{l}\text { Healthy controls } \\
\text { (HC) }\end{array}$} & \multirow{2}{*}{28} & Female & $\begin{array}{c}10 \\
(35.7 \%)\end{array}$ & $\begin{array}{c}37.90 \\
(23.46-52.34)\end{array}$ & $\begin{array}{c}159.40 \\
(152.33-166.47)\end{array}$ & $\begin{array}{c}58.35 \\
(49.61-67.09\end{array}$ & $\begin{array}{c}23.11 \\
(18.95-27.27\end{array}$ \\
\hline & & & Male & $\begin{array}{c}18 \\
(64.3 \%)\end{array}$ & $\begin{array}{c}41.56 \\
(28.47-54.65)\end{array}$ & $\begin{array}{c}176.00 \\
(170.48-181.52)\end{array}$ & $\begin{array}{c}66.45 \\
(58.41-74.49)\end{array}$ & $\begin{array}{c}21.56 \\
(18.24-24.88)\end{array}$ \\
\hline & \multirow{2}{*}{$\begin{array}{l}\text { TB patients } \\
\text { (TB) }\end{array}$} & \multirow{2}{*}{26} & Female & $\begin{array}{c}8 \\
(30.8 \%)\end{array}$ & $\begin{array}{c}35.88 \\
(23.80-47.96)\end{array}$ & $\begin{array}{c}160.38 \\
(154.53-166.23)\end{array}$ & $\begin{array}{c}55.88 \\
(47.85-63.91)\end{array}$ & $\begin{array}{c}21.78 \\
(18.34-25.22)\end{array}$ \\
\hline & & & Male & $\begin{array}{c}18 \\
(69.2 \%)\end{array}$ & $\begin{array}{c}36.33 \\
(26.55-46.11)\end{array}$ & $\begin{array}{c}174.39 \\
(167.22-181.56)\end{array}$ & $\begin{array}{c}73.94 \\
(65.36-82.52)\end{array}$ & $\begin{array}{c}24.41 \\
(21.03-27.79)\end{array}$ \\
\hline \multirow{4}{*}{$\begin{array}{l}\text { Foshan } \\
\text { cohort }\end{array}$} & \multirow{2}{*}{$\begin{array}{l}\text { Healthy controls } \\
\text { (HC) }\end{array}$} & \multirow{2}{*}{17} & Female & $\begin{array}{c}5 \\
(29.4 \%) \\
\end{array}$ & $\begin{array}{c}33.60 \\
(23.36-43.84) \\
\end{array}$ & $\begin{array}{c}159.40 \\
(152.03-166.77) \\
\end{array}$ & $\begin{array}{c}51.60 \\
(44.23-58.97) \\
\end{array}$ & $\begin{array}{c}20.23 \\
(18.63-21.83) \\
\end{array}$ \\
\hline & & & Male & $\begin{array}{c}12 \\
(70.6 \%)\end{array}$ & $\begin{array}{c}31.43 \\
(21.60-41.26)\end{array}$ & $\begin{array}{c}170.50 \\
(162.72-178.28)\end{array}$ & $\begin{array}{c}64.08 \\
(53.48-74.68)\end{array}$ & $\begin{array}{c}22.09 \\
(18.47-25.71)\end{array}$ \\
\hline & \multirow[b]{2}{*}{$\begin{array}{l}\text { TB patients } \\
\text { (TB) }\end{array}$} & \multirow{2}{*}{19} & Female & $\begin{array}{c}8 \\
(42.1 \%) \\
\end{array}$ & $\begin{array}{c}33.88 \\
(21.85-45.91) \\
\end{array}$ & $\begin{array}{c}157.63 \\
(152.05-163.21)\end{array}$ & $\begin{array}{c}54.25 \\
(46.64-61.86) \\
\end{array}$ & $\begin{array}{c}21.84 \\
(18.83-24.85) \\
\end{array}$ \\
\hline & & & Male & $\begin{array}{c}11 \\
(57.9 \%)\end{array}$ & $\begin{array}{c}36.82 \\
(24.86-48.78) \\
\end{array}$ & $\begin{array}{c}174.73 \\
(167.73-181.73) \\
\end{array}$ & $\begin{array}{c}73.82 \\
(63.46-84.18)\end{array}$ & $\begin{array}{c}24.33 \\
(20.15-28.51)\end{array}$ \\
\hline \multirow{4}{*}{$\begin{array}{l}\text { Combined } \\
\text { cohort }\end{array}$} & \multirow{2}{*}{$\begin{array}{l}\text { Healthy controls } \\
\text { (HC) }\end{array}$} & \multirow{2}{*}{45} & Female & $\begin{array}{c}15 \\
(33.3 \%)\end{array}$ & $\begin{array}{c}36.47 \\
(23.49-49.45) \\
\end{array}$ & $\begin{array}{c}159.40 \\
(152.49-166.31) \\
\end{array}$ & $\begin{array}{c}56.10 \\
(47.41-64.79)\end{array}$ & $\begin{array}{c}22.15 \\
(18.43-25.87)\end{array}$ \\
\hline & & & Male & $\begin{array}{c}30 \\
(66.7 \%) \\
\end{array}$ & $\begin{array}{c}37.51 \\
(24.76-50.26) \\
\end{array}$ & $\begin{array}{c}173.27 \\
(165.8-180.74) \\
\end{array}$ & $\begin{array}{c}67.08 \\
(57.51-76.65) \\
\end{array}$ & $\begin{array}{c}22.46 \\
(18.76-26.16) \\
\end{array}$ \\
\hline & \multirow{2}{*}{$\begin{array}{l}\text { TB patients } \\
\text { (TB) }\end{array}$} & \multirow{2}{*}{45} & Female & $\begin{array}{c}16 \\
(35.6 \%) \\
\end{array}$ & $\begin{array}{c}34.88 \\
(23.19-46.57) \\
\end{array}$ & $\begin{array}{c}159.00 \\
(153.30-164.70)\end{array}$ & $\begin{array}{c}55.06 \\
(47.46-62.66) \\
\end{array}$ & $\begin{array}{c}21.81 \\
(18.69-24.93) \\
\end{array}$ \\
\hline & & & Male & $\begin{array}{c}29 \\
(64.4 \%)\end{array}$ & $\begin{array}{c}36.52 \\
(26.07-46.97)\end{array}$ & $\begin{array}{c}174.52 \\
(167.54-181.50)\end{array}$ & $\begin{array}{c}73.90 \\
(64.79-83.01)\end{array}$ & $\begin{array}{c}24.38 \\
(20.75-28.01)\end{array}$ \\
\hline
\end{tabular}




\begin{tabular}{|c|c|c|c|c|c|c|c|c|}
\hline Cohort & Groups & $\begin{array}{l}\text { Sample } \\
\text { size }\end{array}$ & Sex & Number & Age & Height & Weight & BMI \\
\hline \multirow{4}{*}{$\begin{array}{l}\text { Guangzhou } \\
\text { cohort }\end{array}$} & \multirow{2}{*}{$\begin{array}{l}\text { Healthy controls } \\
\text { (HC) }\end{array}$} & \multirow{2}{*}{263} & Female & $\begin{array}{c}86 \\
(32.7 \%) \\
\end{array}$ & $\begin{array}{c}32.98 \\
(20.78-45.18) \\
\end{array}$ & $\begin{array}{c}162.22 \\
(155.05-169.39) \\
\end{array}$ & $\begin{array}{c}55.76 \\
(47.70-63.82) \\
\end{array}$ & $\begin{array}{c}21.31 \\
(17.68-24.94) \\
\end{array}$ \\
\hline & & & Male & $\begin{array}{c}177 \\
(67.3 \%) \\
\end{array}$ & $\begin{array}{c}33.81 \\
(22.50-45.12) \\
\end{array}$ & $\begin{array}{c}174.16 \\
(164.88-182.44) \\
\end{array}$ & $\begin{array}{c}69.46 \\
(60.23-78.69) \\
\end{array}$ & $\begin{array}{c}23.05 \\
(19.27-26.83) \\
\end{array}$ \\
\hline & \multirow{2}{*}{$\begin{array}{l}\text { TB patients } \\
\text { (TB) }\end{array}$} & \multirow{2}{*}{264} & Female & $\begin{array}{c}101 \\
(38.3 \%) \\
\end{array}$ & $\begin{array}{c}36.40 \\
(24.68-48.12) \\
\end{array}$ & $\begin{array}{c}162.79 \\
(156.06-169.52) \\
\end{array}$ & $\begin{array}{c}56.51 \\
(48.29-64.73) \\
\end{array}$ & $\begin{array}{c}21.43 \\
(17.83-25.03) \\
\end{array}$ \\
\hline & & & Male & $\begin{array}{c}163 \\
(61.7 \%) \\
\end{array}$ & $\begin{array}{c}37.71 \\
(19.91-55.51) \\
\end{array}$ & $\begin{array}{c}174.15 \\
(165.30-183.00) \\
\end{array}$ & $\begin{array}{c}71.05 \\
(62.07-80.03) \\
\end{array}$ & $\begin{array}{c}23.63 \\
(19.67-27.59) \\
\end{array}$ \\
\hline \multirow{4}{*}{$\begin{array}{l}\text { Shenzhen } \\
\text { cohort }\end{array}$} & \multirow{2}{*}{$\begin{array}{l}\text { Healthy controls } \\
\text { (HC) }\end{array}$} & \multirow{2}{*}{1445} & Female & $\begin{array}{c}563 \\
(39.0 \%) \\
\end{array}$ & $\begin{array}{c}34.26 \\
(17.58-49.56) \\
\end{array}$ & $\begin{array}{c}161.55 \\
(154.40-168.70) \\
\end{array}$ & $\begin{array}{c}56.76 \\
(48.91-64.61) \\
\end{array}$ & $\begin{array}{c}21.90 \\
(18.16-25.64) \\
\end{array}$ \\
\hline & & & Male & $\begin{array}{c}882 \\
(61.0 \%) \\
\end{array}$ & $\begin{array}{c}37.18 \\
(17.00-56.75) \\
\end{array}$ & $\begin{array}{c}173.82 \\
(165.31-182.33) \\
\end{array}$ & $\begin{array}{c}70.08 \\
(61.10-79.06) \\
\end{array}$ & $\begin{array}{c}23.35 \\
(19.64-27.06) \\
\end{array}$ \\
\hline & \multirow{2}{*}{$\begin{array}{l}\text { TB patients } \\
\text { (TB) }\end{array}$} & \multirow{2}{*}{1533} & Female & $\begin{array}{c}553 \\
(36.1 \%) \\
\end{array}$ & $\begin{array}{c}35.18 \\
(21.38-49.18) \\
\end{array}$ & $\begin{array}{c}161.19 \\
(154.22-168.16) \\
\end{array}$ & $\begin{array}{c}56.14 \\
(47.95-64.33) \\
\end{array}$ & $\begin{array}{c}21.72 \\
(18.07-25.39) \\
\end{array}$ \\
\hline & & & Male & $\begin{array}{c}980 \\
(63.9 \%) \\
\end{array}$ & $\begin{array}{c}37.23 \\
(22.93-52.69) \\
\end{array}$ & $\begin{array}{c}173.96 \\
(164.55-182.37) \\
\end{array}$ & $\begin{array}{c}70.28 \\
(61.29-79.27) \\
\end{array}$ & $\begin{array}{c}23.40 \\
(19.54-27.26) \\
\end{array}$ \\
\hline \multirow{4}{*}{$\begin{array}{l}\text { Foshan } \\
\text { cohort }\end{array}$} & \multirow[b]{2}{*}{$\begin{array}{l}\text { Healthy controls } \\
\text { (HC) }\end{array}$} & \multirow{2}{*}{1679} & Female & $\begin{array}{c}574 \\
(34.2 \%) \\
\end{array}$ & $\begin{array}{c}29.06 \\
(19.90-45.35) \\
\end{array}$ & $\begin{array}{c}161.16 \\
(154.18-168.14)\end{array}$ & $\begin{array}{c}56.57 \\
(48.51-64.63) \\
\end{array}$ & $\begin{array}{c}21.91 \\
(18.21-25.61) \\
\end{array}$ \\
\hline & & & Male & $\begin{array}{c}1105 \\
(65.8 \%) \\
\end{array}$ & $\begin{array}{c}31.10 \\
(20.10-46.70) \\
\end{array}$ & $\begin{array}{c}173.98 \\
(165.50-182.46)\end{array}$ & $\begin{array}{c}70.41 \\
(61.55-79.27) \\
\end{array}$ & $\begin{array}{c}23.42 \\
(19.71-27.13) \\
\end{array}$ \\
\hline & \multirow{2}{*}{$\begin{array}{l}\text { TB patients } \\
\text { (TB) }\end{array}$} & \multirow{2}{*}{1328} & Female & $\begin{array}{c}437 \\
(32.9 \%) \\
\end{array}$ & $\begin{array}{c}35.11 \\
(21.21-49.01) \\
\end{array}$ & $\begin{array}{c}161.29 \\
(154.48-168.10)\end{array}$ & $\begin{array}{c}55.76 \\
(47.71-63.81) \\
\end{array}$ & $\begin{array}{c}21.55 \\
(17.94-25.16) \\
\end{array}$ \\
\hline & & & Male & $\begin{array}{c}891 \\
(67.1 \%) \\
\end{array}$ & $\begin{array}{c}37.95 \\
(23.18-49.08) \\
\end{array}$ & $\begin{array}{c}174.44 \\
(166.24-182.64) \\
\end{array}$ & $\begin{array}{c}69.98 \\
(60.85-79.11) \\
\end{array}$ & $\begin{array}{c}23.16 \\
(19.35-26.97) \\
\end{array}$ \\
\hline \multirow{4}{*}{$\begin{array}{l}\text { Combined } \\
\text { cohort }\end{array}$} & \multirow{2}{*}{$\begin{array}{l}\text { Healthy controls } \\
\text { (HC) }\end{array}$} & \multirow{2}{*}{3387} & Female & $\begin{array}{c}1223 \\
(36.1 \%)\end{array}$ & $\begin{array}{c}31.73 \\
(19.00-47.45)\end{array}$ & $\begin{array}{c}161.41 \\
(154.34-168.48) \\
\end{array}$ & $\begin{array}{c}56.60 \\
(48.64-64.56) \\
\end{array}$ & $\begin{array}{c}21.86 \\
(18.14-25.58)\end{array}$ \\
\hline & & & Male & $\begin{array}{c}2164 \\
(63.2 \%)\end{array}$ & $\begin{array}{c}33.80 \\
(19.20-51.78)\end{array}$ & $\begin{array}{c}173.93 \\
(165.45-182.41)\end{array}$ & $\begin{array}{c}70.2 \\
(61.26-79.14)\end{array}$ & $\begin{array}{c}23.36 \\
(19.64-27.08)\end{array}$ \\
\hline & \multirow{2}{*}{$\begin{array}{l}\text { TB patients } \\
\text { (TB) }\end{array}$} & \multirow{2}{*}{3125} & Female & $\begin{array}{c}1091 \\
(34.91 \%)\end{array}$ & $\begin{array}{c}35.26 \\
(21.32-49.08) \\
\end{array}$ & $\begin{array}{c}161.38 \\
(154.49-168.27) \\
\end{array}$ & $\begin{array}{c}56.02 \\
(47.89-64.15) \\
\end{array}$ & $\begin{array}{c}21.63 \\
(17.99-25.25) \\
\end{array}$ \\
\hline & & & Male & $\begin{array}{c}2034 \\
(65.09 \%)\end{array}$ & $\begin{array}{c}37.58 \\
(23.04-51.16)\end{array}$ & $\begin{array}{c}174.18 \\
(165.83-182.53)\end{array}$ & $\begin{array}{c}70.21 \\
(61.16-79.26)\end{array}$ & $\begin{array}{c}23.32 \\
(19.47-27.17)\end{array}$ \\
\hline
\end{tabular}

1524 Supplementary Table 2. Demographic characteristics of active TB patients (TB)

1525 and healthy controls (HC) for SNP analyses. 


\begin{tabular}{|c|c|c|c|c|c|c|}
\hline \multirow{2}{*}{$\begin{array}{c}\text { Chromosome } 21 \\
\text { position }\end{array}$} & \multirow{2}{*}{ SNP ID } & \multirow{2}{*}{ Genotype } & \multirow{2}{*}{$\begin{array}{c}\mathrm{HC} \\
\mathrm{NO}(\%)\end{array}$} & \multirow{2}{*}{$\begin{array}{c}\text { TB } \\
\text { NO }(\%)\end{array}$} & \multicolumn{2}{|c|}{ Additive } \\
\hline & & & & & $P$ & OR $(95 \% \mathrm{Cl})$ \\
\hline \multirow[t]{3}{*}{ chr21:33343393 } & rs2257167 & GG & $96(36.50)$ & $105(39.77)$ & 0.28 & $1.32(0.80-2.23)$ \\
\hline & & GC & $121(46.01)$ & $121(45.83)$ & 0.45 & $1.21(0.75-1.99)$ \\
\hline & & $\mathrm{CC}$ & $46(17.49)$ & 38 (14.39) & Ref. & Ref. \\
\hline \multirow[t]{3}{*}{ chr21:33355024 } & rs1041868 & GG & $108(41.06)$ & $112(42.42)$ & 0.59 & $1.16(0.68-1.96)$ \\
\hline & & $\mathrm{GA}$ & $116(44.11)$ & $117(44.32)$ & 0.66 & $1.12(0.67-1.90)$ \\
\hline & & $A A$ & $39(14.83)$ & $35(13.26)$ & Ref. & Ref. \\
\hline \multirow[t]{3}{*}{ chr21:33326436 } & rs4408796 & $\mathrm{CC}$ & $142(54.00)$ & $143(54.17)$ & 0.89 & $0.95(0.48-1.89)$ \\
\hline & & CG & $103(39.16)$ & $102(38.63)$ & 0.86 & $0.94(0.47-1.89)$ \\
\hline & & GG & $18(6.84)$ & $19(7.20)$ & Ref. & Ref. \\
\hline \multirow[t]{3}{*}{ chr21:33331291 } & rs 13050445 & $\mathrm{CC}$ & $130(49.43)$ & $132(50.00)$ & 0.96 & $1.02(0.54-1.90)$ \\
\hline & & CT & $110(41.83)$ & 109 (41.29) & 0.98 & $0.99(0.52-1.87)$ \\
\hline & & $\mathrm{TT}$ & $23(8.74)$ & $23(8.71)$ & Ref. & Ref. \\
\hline \multirow[t]{3}{*}{ chr21:33331895 } & rs2252930 & $\mathrm{CC}$ & $256(97.34)$ & 257 (97.35) & - & - \\
\hline & & CG & $7(2.66)$ & $7(2.65)$ & - & - \\
\hline & & GG & $0(0.00)$ & $0(0.00)$ & Ref. & Ref. \\
\hline \multirow[t]{3}{*}{ chr21:33332014 } & rs2252931 & GG & $102(38.78)$ & $105(39.77)$ & 0.83 & $1.06(0.60-1.90)$ \\
\hline & & GA & $131(49.81)$ & $130(49.24)$ & 0.93 & $1.03(0.58-1.81)$ \\
\hline & & $A A$ & $30(11.41)$ & $29(10.98)$ & Ref. & Ref. \\
\hline \multirow[t]{3}{*}{ chr21:33340113 } & rs2253923 & AA & $123(46.77)$ & $122(46.21)$ & 0.98 & $0.99(0.52-1.89)$ \\
\hline & & AT & $118(44.87)$ & $120(45.45)$ & 0.96 & $1.02(0.53-1.94)$ \\
\hline & & $\mathrm{TT}$ & $22(8.36)$ & $22(8.33)$ & Ref. & Ref. \\
\hline \multirow[t]{3}{*}{ chr21:33351188 } & rs2243599 & $\mathrm{TT}$ & $126(47.90)$ & $127(48.11)$ & 0.92 & $0.97(0.54-1.74)$ \\
\hline & & TG & $110(41.83)$ & $109(41.29)$ & 0.88 & $0.96(0.53-1.73)$ \\
\hline & & GG & $27(10.27)$ & $28(10.60)$ & Ref. & Ref. \\
\hline \multirow[t]{3}{*}{ chr21:33353924 } & rs2254180 & $\mathrm{TT}$ & $145(55.13)$ & $144(54.55)$ & 0.98 & $0.99(0.49-2.02)$ \\
\hline & & TC & $101(38.40)$ & $103(39.01)$ & 0.96 & $1.02(0.49-2.11)$ \\
\hline & & $\mathrm{CC}$ & $17(6.46)$ & $17(6.44)$ & Ref. & Ref. \\
\hline \multirow[t]{3}{*}{ chr21:33355181 } & rs2254315 & $\mathrm{CC}$ & $251(95.44)$ & $253(95.83)$ & - & - \\
\hline & & $\mathrm{CT}$ & $12(4.56)$ & $11(4.17)$ & - & - \\
\hline & & $\mathrm{TT}$ & $0(0.00)$ & $0(0.00)$ & Ref. & Ref. \\
\hline
\end{tabular}

- Discovery cohort (Guangzhou), Chromosome 21 IFNAR1 locus (GRCh38.p12, 33324395 - 33359864 ).

- $P$-value $(P)$ was calculated comparing active TB patients (TB) group to healthy controls $(\mathrm{HC})$ group in allele distribution.

1528 Supplementary Table 3. Association of Ifnar1 SNPs and TB susceptibility in a

1529 discovery cohort. 


\begin{tabular}{|c|c|c|c|c|c|c|c|c|c|c|c|c|c|}
\hline \multirow[b]{2}{*}{ SNP ID } & \multirow{2}{*}{\multicolumn{2}{|c|}{ Cohort }} & \multirow[b]{2}{*}{ Genotype } & \multirow{2}{*}{$\begin{array}{l}\text { HC } \\
\text { NO } \\
(\%) \\
\end{array}$} & \multirow{2}{*}{\begin{tabular}{|l|} 
TB \\
NO \\
$(\%)$ \\
\end{tabular}} & \multicolumn{2}{|c|}{ Multiplicative } & \multicolumn{2}{|c|}{ Additive } & \multicolumn{2}{|c|}{ Dominant } & \multicolumn{2}{|c|}{ Recessive } \\
\hline & & & & & & $P$ & $\begin{array}{c}\text { OR } \\
(95 \% \mathrm{Cl})\end{array}$ & $P$ & $\begin{array}{c}\text { OR } \\
(95 \% \mathrm{Cl})\end{array}$ & $P$ & $\begin{array}{c}\text { OR } \\
(95 \% \mathrm{Cl})\end{array}$ & $P$ & $\begin{array}{c}\text { OR } \\
(95 \% \mathrm{Cl})\end{array}$ \\
\hline \multirow{9}{*}{ rs2257167 } & \multirow{6}{*}{ Validation } & \multirow{3}{*}{$\begin{array}{c}\text { Shenzhen } \\
\text { cohort }\end{array}$} & GG & $\begin{array}{c}515 \\
(35.64)\end{array}$ & $\begin{array}{c}631 \\
(41.16)\end{array}$ & \multirow{3}{*}{0.0002} & \multirow{3}{*}{$\begin{array}{c}1.22 \\
(1.10-1.36)\end{array}$} & 0.0003 & $\begin{array}{c}1.50 \\
(1.21-1.87)\end{array}$ & \multirow{3}{*}{0.0024} & \multirow{3}{*}{$\begin{array}{c}1.36 \\
(1.12-1.67)\end{array}$} & \multirow{3}{*}{0.002} & \multirow{3}{*}{$\begin{array}{c}1.26 \\
(1.09-1.47)\end{array}$} \\
\hline & & & GC & $\begin{array}{c}681 \\
(47.13)\end{array}$ & $\begin{array}{c}699 \\
(45.60)\end{array}$ & & & 0.03 & $\begin{array}{c}1.26 \\
(1.02-1.56)\end{array}$ & & & & \\
\hline & & & CC & $\begin{array}{c}249 \\
(17.23)\end{array}$ & $\begin{array}{c}203 \\
(13.24)\end{array}$ & & & Ref. & Ref. & & & & \\
\hline & & \multirow{3}{*}{$\begin{array}{c}\text { Foshan } \\
\text { cohort }\end{array}$} & GG & $\begin{array}{c}605 \\
(36.03)\end{array}$ & $\begin{array}{c}542 \\
(40.81) \\
\end{array}$ & & & 0.0002 & $\begin{array}{c}1.52 \\
(1.22-1.90)\end{array}$ & \multirow{3}{*}{0.001} & \multirow{3}{*}{$\begin{array}{c}1.41 \\
(1.15-1.74)\end{array}$} & \multirow{3}{*}{0.007} & \multirow{3}{*}{$\begin{array}{c}1.22 \\
(1.06-1.42)\end{array}$} \\
\hline & & & GC & $\begin{array}{c}792 \\
(47.17) \\
\end{array}$ & $\begin{array}{c}620 \\
(46.69) \\
\end{array}$ & 0.0003 & $\begin{array}{c}1.21 \\
(1.09-1.35)\end{array}$ & 0.01 & $\begin{array}{c}1.33 \\
(1.07-1.66)\end{array}$ & & & & \\
\hline & & & CC & $\begin{array}{c}282 \\
(16.80)\end{array}$ & $\begin{array}{c}166 \\
(12.50) \\
\end{array}$ & & & Ref. & Ref. & & & & \\
\hline & \multirow{3}{*}{\multicolumn{2}{|c|}{$\begin{array}{c}\text { Combined } \\
\text { cohort }\end{array}$}} & GG & $\begin{array}{c}1120 \\
(35.85)\end{array}$ & $\begin{array}{c}1173 \\
(41.00) \\
\end{array}$ & \multirow{3}{*}{0.000} & \multirow{3}{*}{$\begin{array}{c}1.22 \\
(1.13-1.31)\end{array}$} & 0.000 & $\begin{array}{c}1.51 \\
(1.29-1.76)\end{array}$ & \multirow{3}{*}{0.000} & \multirow{3}{*}{$\begin{array}{c}1.38 \\
(1.20-1.60)\end{array}$} & \multirow{3}{*}{0.000} & \multirow{3}{*}{$\begin{array}{c}1.24 \\
(1.12-1.38)\end{array}$} \\
\hline & & & GC & $\begin{array}{c}1473 \\
(47.15)\end{array}$ & $\begin{array}{c}1319 \\
(46.10) \\
\end{array}$ & & & 0.001 & $\begin{array}{c}1.29 \\
(1.11-1.51)\end{array}$ & & & & \\
\hline & & & cc & $\begin{array}{c}531 \\
(17.00)\end{array}$ & $\begin{array}{c}369 \\
(12.90) \\
\end{array}$ & & & Ref. & Ref. & & & & \\
\hline \multirow{9}{*}{ rs1041868 } & \multirow{6}{*}{ Validation } & & GG & $\begin{array}{c}521 \\
(36.05)\end{array}$ & $\begin{array}{c}560 \\
(36.53) \\
\end{array}$ & & & 0.38 & $\begin{array}{c}1.10 \\
(0.44-1.37)\end{array}$ & & & & \\
\hline & & $\begin{array}{c}\text { Shenzhen } \\
\text { cohort }\end{array}$ & GA & $\begin{array}{c}682 \\
(47.20)\end{array}$ & $\begin{array}{c}737 \\
(48.08) \\
\end{array}$ & 0.47 & $\begin{array}{c}1.04 \\
(0.94-1.15)\end{array}$ & 0.33 & $\begin{array}{c}1.11 \\
(0.90-1.36)\end{array}$ & 0.31 & $\begin{array}{c}1.11 \\
(0.91-1.35)\end{array}$ & 0.79 & $\begin{array}{c}1.02 \\
(0.88-1.19)\end{array}$ \\
\hline & & & AA & $\begin{array}{c}242 \\
(16.75)\end{array}$ & $\begin{array}{c}236 \\
(15.39)\end{array}$ & & & Ref. & Ref. & & & & \\
\hline & & & GG & $\begin{array}{c}563 \\
(33.53)\end{array}$ & $\begin{array}{c}461 \\
(34.71)\end{array}$ & & & 0.89 & $\begin{array}{c}0.98 \\
(0.80-1.22)\end{array}$ & & & & \\
\hline & & $\begin{array}{l}\text { Foshan } \\
\text { cohort }\end{array}$ & GA & $\begin{array}{c}837 \\
(49.85)\end{array}$ & $\begin{array}{c}635 \\
(47.82) \\
\end{array}$ & 0.89 & $\begin{array}{c}1.01 \\
(0.91-1.12)\end{array}$ & 0.37 & $\begin{array}{c}0.91 \\
(0.75-1.12)\end{array}$ & 0.54 & $\begin{array}{c}0.94 \\
(0.78-1.14)\end{array}$ & 0.50 & $\begin{array}{c}1.05 \\
(0.91-1.23)\end{array}$ \\
\hline & & & AA & $\begin{array}{c}279 \\
(16.62)\end{array}$ & $\begin{array}{c}232 \\
(17.47)\end{array}$ & & & Ref. & Ref. & & & & \\
\hline & & & GG & $\begin{array}{c}1084 \\
(34.70)\end{array}$ & $\begin{array}{c}1021 \\
(35.69)\end{array}$ & & & 0.54 & $\begin{array}{c}1.05 \\
(0.45-1.24)\end{array}$ & & & & \\
\hline & $\begin{array}{r}\text { Comb } \\
\text { cohs }\end{array}$ & ined & GA & $\begin{array}{c}1519 \\
(48.62)\end{array}$ & $\begin{array}{c}1372 \\
(47.96) \\
\end{array}$ & 0.47 & $\begin{array}{c}1.03 \\
(0.96-1.11)\end{array}$ & 0.94 & $\begin{array}{c}1.01 \\
(0.87-1.31)\end{array}$ & 0.74 & $\begin{array}{c}1.02 \\
(0.89-1.17)\end{array}$ & 0.42 & $\begin{array}{c}1.04 \\
(0.94-1.16)\end{array}$ \\
\hline & & & AA & $\begin{array}{c}521 \\
(16.68)\end{array}$ & $\begin{array}{c}468 \\
(16.35)\end{array}$ & & & Ref. & Ref. & & & & \\
\hline
\end{tabular}

1532 Supplementary Table 4. The association of Ifnar1 SNP rs2257167 or rs1041868

1533 and TB susceptibility in Shenzhen and Foshan cohorts. 


\begin{tabular}{|c|c|c|}
\hline Primers & Sequence & \\
\hline A. muciniphila & For: 5'-CAGCACGTGAAGGTGGGGAC-3' & Rev:5'-CCTTGCGGTTGGCTTCAGAT-3' \\
\hline \multirow{2}{*}{ B. vulgatus } & For: 5'-CGGGCTTAAATTGCAGATGA-3' & Rev:5'-CATGCAGCACCTTCACAGAT-3' \\
\hline & \multicolumn{2}{|c|}{ Probe: FAM-CCAACCTGCCGACAACACTGGGATA-TAM } \\
\hline \multirow{2}{*}{ 16S rDNA } & For: 5'-CGGTGAATACGTTCCCGG-3' & Rev:5'-TACGGCTACCTTGTTACGACTT-3' \\
\hline & \multicolumn{2}{|l|}{ Probe: FAM-CTTGTACACACCGCCCGTC-MGB } \\
\hline $\mathrm{h} / \mathrm{FN}-\beta$ & For: 5'-CCTACAAAGAAGCAGCAA-3' & Rev:5'-TCCTCAGGGATGTCAAAG-3' \\
\hline hISG15 & For: 5'-CGCAGATCACCCAGAAGATCG-3' & Rev:5'-TTCGTCGCATTTGTCCACCA-3' \\
\hline GAPDH & For: 5'-CGGAGTCAACGGATTTGGTC-3' & Rev:5'-GACAAGCTTCCCGTTCTCAG-3' \\
\hline $\mathrm{m} / \mathrm{FN}-\beta$ & For: 5'-AGATCAACCTCACCTACAGG-3' & Rev:5'-TCAGAAACACTGTCTGCTGG-3' \\
\hline $\mathrm{m} / \mathrm{SG} 15$ & For: 5'-TCCATGACGGTGTCAGAACT-3' & Rev:5'-GACCCAGACTGGAAAGGGTA-3' \\
\hline$m G A P D H$ & For: 5'-GAAGGGCTCATGACCACAGT-3' & Rev:5'-GGATGCAGGGATGATGTTCT-3' \\
\hline FAT & For: 5'-GCGAATGGCCTGATGAAACC-3' & Rev:5'-CCACCGTTTCTTTTGCATGGG-3' \\
\hline Amuc0201 & For: 5'-ATGGCTATTGATCCCAAATT-3' & Rev:5'-TTAGAGCGGAATGTTGCCGT-3' \\
\hline Amuc1507 & For: 5'-ATGGACGCAGTATTATTGTTTTT-3' & Rev:5'-TTATTTGTTCAGCTCCGCAA-3' \\
\hline Amuc1327 & For: 5'-ATGACCGACCGCAGAATTGT-3' & Rev:5'-TCAGGCGAATCGTTTCACGA-3' \\
\hline Amuc0994 & For: 5'-ATGCAAAAGTTAGCAGGTAAAA-3' & Rev:5'-CTACATCGTCATGCCTCCGT-3' \\
\hline Amuc1918 & For: 5'-ATGGCATGTGGAAACCAAAG-3' & Rev:5'-TCAAGCATCCTGAAGGCCGA-3' \\
\hline Amuc1642 & For: 5'-ATGTCAAGTAAATTACTCGAAGG-3' & Rev:5'-TTACATTCCCATGATCTGATAAC-3' \\
\hline Amuc1725 & For: 5'-ATGGAACATGCCAGCAACAA-3' & Rev:5'-TTATGCGATTTCAGCGGTTTT-3' \\
\hline BVU1463 & For: 5'-ATGATACAAAAGGTTCTAATTGC-3' & Rev:5'-TCAGATTCTCAACACTCCTTTC-3' \\
\hline BVU3104 & For: 5'-ATGATAAAGAAAGTATTGATTGCA-3' & Rev:5'-TCAGATTCTCAATACTCCTTTCTG-3' \\
\hline BVU3989 & For: 5'-ATGAAAGCATTTGTATTCCCC-3' & Rev:5'-TCAAGCAATACCGTGTGCGT-3' \\
\hline BVU1050 & For: 5'-ATGGAATTAAAAAGAGTAGTAGTAA-3' & Rev:5'-TTATTCAGCATATTTTTTTACG-3' \\
\hline BVU0384 & For: 5'-ATGGGATTATTAACAGGAAAGAC-3' & Rev:5'-TTACATGTTCATACCGCCGT-3' \\
\hline BVU1011 & For: 5'-ATGAAATACGCACTAGTAACAGG-3' & Rev:5'-TTATGTATAGATTCCTCCATTGATG-3' \\
\hline BVU0098 & For: 5'-ATGTTGAAACAGAAGACATTAAAAG-3' & Rev:5'-TTATTTATTTTTTTACTATTTGTGCC-3' \\
\hline BVU1026 & For: 5'-ATGCTGCTGGAGAACTTTTATA-3' & Rev:5'-TCATTGATACTTACTTGCCATC-3' \\
\hline BVU1986 & For: 5'-ATGAGTTACAATTTGTTGAAAGG-3' & Rev:5'-TTATCCGTAAATGATGTTTCC-3' \\
\hline BVU2557 & For: 5'-ATGATGAGTGAGAATAAAGTAGGAA-3' & Rev:5'-CTAAATAAATATCACCTTACTGCG-3' \\
\hline BVU0009 & For: 5'-ATGATAAAAGAAAACTTTATCAAGC-3' & Rev:5'-TTATTCTTCGGTAATACTGTTATAT-3' \\
\hline BVU1484 & For: 5'-ATGAGTAGTTCTTTCTTATCTGTCC-3' & Rev:5'-TTATTCTTCGTACATCTTTTCTATT-3' \\
\hline BVU3027 & For: 5'-ATGGAACTAGAACAGAGTTTTATTTG-3' & Rev:5'-TTATTCTTTTATATCTTGGTACAAG-3' \\
\hline BVU3870 & For: 5'-ATGGAGCAGAGTTTTATTGC-3' & Rev:5'-TTATTTTACTTCCTGATATAAGAAA-3' \\
\hline
\end{tabular}

1535 Supplementary Table 5. Primers used in this study. 


\section{Supplementary Files}

This is a list of supplementary files associated with this preprint. Click to download.

- STROBEchecklistcohort.pdf

- nreditorialpolicychecklist.pdf

- nrreportingsummary.pdf

- AblankversionofthelnformedConsentofGuangzhouShenzhenandFoshancohorts.pdf

- TheethicalboardapprovalsofGuangzhouShenzhenandFoshancohorts.pdf 